\title{
NMR survey of the reflected Brownian motion
}

\author{
Denis S. Grebenkov* \\ Dipartimento di Scienze Fisiche, Università di Napoli Federico II \\ Complesso universitario Monte S. Angelo, Via Cintia, 80126 Naples, Italy \\ Unité de Recherche en Résonance Magnétique Médicale, \\ C.N.R.S. - Université Paris-Sud XI, 91405 Orsay, FRANCE
}

(Dated: March 26, 2006)

\begin{abstract}
Restricted diffusion is a common feature of many physico-chemical, biological and industrial processes. Nuclear magnetic resonance techniques are often used to survey the atomic or molecular motion in confining media by applying inhomogeneous magnetic fields to encode the trajectories of spin-bearing particles. The diversity and complexity of diffusive NMR phenomena, observed in experiments, result from the specific properties of the reflected Brownian motion. Here we focus on mathematical aspects of this stochastic process as well as on its physical interpretations and practical applications. The main achievements in this field, from Hahn's discovery of spin echoes till nowadays, are presented in a unified mathematical language. A long-standing problem of restricted diffusion under arbitrary magnetic field is reformulated in terms of multiple correlation functions of the reflected Brownian motion. Many classical results are retrieved, extended and critically discussed within an original theoretical approach.
\end{abstract}

Keywords: NMR, restricted diffusion, confining media, reflected Brownian motion

\section{Contents}

I. Introduction

II. A historical overview

A. Studying free diffusion by NMR

B. Bloch-Torrey equation

C. Effect of a geometrical restriction

D. Narrow-pulse approximation

E. Restricted diffusion in simple domains

F. Gaussian phase approximation

G. Non-Gaussian behavior

H. Studying porous materials

I. Nonlinear magnetic fields

J. Matrix formalisms

III. Multiple correlation function approach

A. General case

B. Application to spin echoes

C. Temporal profiles of the magnetic field

D. Spatial profiles of the magnetic field

1. Linear gradient

2. Parabolic magnetic field

E. Basic confining domains

1. Slab

2. Cylinder

3. Sphere

IV. Slow diffusion regime $(p \ll 1)$

A. Leading term of the second moment

B. Higher order moments

C. Correction term to the second moment

D. Specific temporal profiles

E. Discussion on the correction term

V. Motional narrowing regime $(p \gg 1)$

A. Leading terms
B. Corrections to the leading term

1. Stejskal-Tanner temporal profile

2. Oscillating temporal profile

VI. Discussion

A. Numerical implementation

B. Odd order moments

1. First moment

2. Antisymmetric temporal profiles

C. Cumulant expansion

D. Apparent diffusion coefficient

E. Narrow-pulse approximation

F. Localization regime

G. Unrestricted diffusion

VII. Conclusion

Acknowledgments

A. Fourth moment in the slow diffusion regime

1. First order contribution

2. Second order contribution

B. Laplace transform technique

C. Fourth moment in the motional narrowing regime 36

D. Parabolic magnetic field

1. Cylinder

2. Sphere

E. Multiple propagator approach

F. Even order moments in the narrow-pulse approximation

List of symbols

References

*Electronic address: denis.grebenkov@polytechnique.edu 


\section{INTRODUCTION}

A particle diffusing in a confining medium is a general model for a number of physical, biological and industrial processes. It may describe organic molecules or metabolites in brain tissue (Le Bilan, 2003; Nicholson, 1985,2001 ), reactive species near porous catalysts (Coppens, 1999; Sahimi et al., 1990), ions near rough electrodes or cellular membranes (Halsey and Leibig, 1992; Nyikos and Pajkossy, 1986; Sapoval, 1994, 1996), oxygen in human lungs (Felici et al., 2004, 2005; Grebenkov et al., 2005; Weibel, 1984), water molecules in cements or rocks (Halperin et al., 1994; Nestle et al., 2001; Plassais et al., 2003; Song, 2000), etc. When such a particle encounters an interface, they may interact in different ways, depending on their physical or chemical properties. In general, the interaction at the microscopic level can be represented in terms of "reflection" and "absorption" at the interface. In the former case, the particle does not change its state and continues to diffuse in the bulk. In the latter case, the motion of the particle is terminated, either by absorption on or transfer through the interface, or by chemical transformation into another particle, or by surface relaxation in nuclear magnetic resonance (NMR) experiment, etc. ${ }^{1}$ As a consequence, the erratic random trajectory of a labelled particle is additionally complicated by multiple reflections on the interface.

From the mathematical point of view, this stochastic process is known as (partially) reflected Brownian motion (Bass, 1998; Freidlin, 1985; Grebenkov, 2006; Itô and McKean, 1965; Port and Stone, 1978; Revuz and Yor, 1999; Yor, 2001). It can be rigorously constructed as a solution of the stochastic differential equation accounting for the reflections on the boundary of a confining domain. Although still governed by Laplacian field, this Brownian process presents a lot of new and interesting features due to the geometrical restriction. Its consequences have been marked in domains as different as physiology [diffusional screening in mammal lungs, Felici et al. (2005)], electrochemistry [e.g., constant phase angle frequency regime of the spectroscopic impedance of a rough metallic electrode, Armstrong and Burnham (1976); de Levie (1965); Grebenkov (2006d); Halsey and Leibig (1992); Nyikos and Pajkossy (1985); Sapoval (1987)] or nuclear magnetic resonance [e.g., motional narrowing regime, see below].

The NMR technique is of particular interest since it presents a way to "label" or "encode" Brownian trajectories of spin-bearing particles by magnetic fields. ${ }^{2}$ To

1 In two last examples, the particle can still diffuse, but its state is changed, for example, the nuclear magnetization is lost. Since this particle does not contribute to the process any more, it can be thought as being absorbed.

2 A detailed introduction to the basic principles of NMR can be found in (Abragam, 1961; Callaghan, 1991). A special emphasis on diffusive motion in porous media and its applications is placed illustrate the idea, let us consider nuclei of spin $1 / 2$, e.g., water protons. Under a constant magnetic field $B_{0}$, these nuclei have two states with energies $\mu B_{0}$ and $-\mu B_{0}$ respectively, $\mu$ being the nuclear magnetic moment. ${ }^{3}$ The energy difference corresponds to a resonant or Larmor frequency $2 \mu B_{0} / \hbar=\gamma B_{0}, 2 \pi \hbar$ being the Planck's constant, and $\gamma=2 \mu / \hbar$ is called the nuclear gyromagnetic ratio. ${ }^{4}$ At thermal equilibrium, a difference of state populations creates a magnetization which is oriented along the direction of the magnetic field (traditionally, it is denoted as the axis $z$ ). An application, during a short time, of a periodic magnetic field $B_{1}$ rotating in the transverse plane $x y$ with the Larmor frequency $\gamma B_{0}$ flips the spin magnetizations into this plane. When this so-called $90^{\circ}$ radio-frequency (RF) pulse is turned off, the spin magnetizations precess with the same Larmor frequency $\gamma B_{0}$ in the transverse plane and relax to their equilibrium states (parallel to the axis $z$ ).

If the magnetic field $B_{0}$ is not spatially homogeneous, some spins precess more rapidly than others, depending on their location in the bulk. Consequently, application of a time-dependent inhomogeneous magnetic field $B_{t}(\mathbf{r})$, directed along the $z$ axis, can be used to encode the positions of nuclei. The total phase accumulated along the trajectory $\mathbf{r}(t)$ of a nucleus during time from 0 to $T$ is then

$$
\varphi=\gamma \int_{0}^{T} d t B_{t}(\mathbf{r}(t))
$$

The macroscopic signal $E$ measured at time $T$ is formed by the entire ensemble of the spins diffusing in the confining domain $\Omega$. The individual transverse magnetizations, conveniently written in a complex form $e^{i \varphi}$, have thus to be averaged over this ensemble. In most experiments, the number of spins is so large that one can average over all possible trajectories of the reflected Brownian motion $\mathbf{r}(t)$

$$
E=\mathbb{E}\left\{e^{i \varphi}\right\},
$$

$\varphi$ being considered here as a functional of $\mathbf{r}(t)$. For definiteness, the signal in (2) is normalized to be 1 if $B_{t}(\mathbf{r}) \equiv 0$. Note that the expectation $\mathbb{E}$ includes the average over starting points with a given initial density $\rho(\mathbf{r})$ of spins distributed in the bulk. Since each Brownian trajectory is "weighted" by the functional (1), some parts of this complex process can be emphasized by varying the temporal and spatial dependencies of the applied magnetic field $B_{t}(\mathbf{r})$. One thus disposes of a powerful experimental tool to survey the reflected Brownian motion. Studying the attenuation of the macroscopic signal

in (Blümich, 2000; Kimmich, 1997; Klafter and Drake, 1989).

3 For protons, $\mu=1.41060671 \cdot 10^{-26} \mathrm{~J} / \mathrm{T}$.

4 For example, $\gamma=2.675 \cdot 10^{8} \mathrm{rad} \mathrm{T}^{-1} \mathrm{~s}^{-1}$ for protons, and $\gamma=$ $2.038 \cdot 10^{8} \operatorname{rad~T}^{-1} \mathrm{~s}^{-1}$ for helium-3. 
$E$ in different magnetic fields, one can retrieve useful information about the diffusive motion itself (e.g., the diffusion coefficient) and the confining geometry. For this reason, the NMR technique is widely used to probe complex morphology of natural and artificial materials (e.g., rocks, soils, cements, colloids) or biological tissues (e.g., brain, lung, bones, kidney).

In this review, we reveal and discuss main theoretical achievements in this field, from Hahn's discovery of spin echoes in 1950 till nowadays. The actual understanding of diffusive phenomena results from an intensive work of hundreds of brilliant scientists during half a century. Different theoretical approaches and mathematical concepts were employed in this investigation: random walks and stochastic processes, partial differential equations, density matrices, Green functions, Laplace operator eigenbasis, spectral and Fourier analysis, random fields, etc. If the original Hahn's work described the simplest case of unrestricted diffusion in a time-independent linear magnetic field gradient, further progress mainly consisted of extending his results to include, for instance, geometrical restriction of diffusing nuclei or temporal dependence and spatial inhomogeneity of the magnetic field. Each technique was focused on a particular extension. For instance, Stejskal and Tanner included an arbitrary temporal dependence for unrestricted diffusion, while Robertson and later Neuman studied restricted diffusion in simple domains for specific temporal and spatial profiles. The diversity of theoretical approaches developed during the last decades made difficult comparison between them and understanding of the fundamental properties and common features of the reflecting Brownian motion. We believe that a single mathematical approach to diffusive NMR phenomena will clarify the state of the art and can bring new ideas in this field. A "multiple correlation function" approach has been developed and used throughout this review to retrieve, discuss and extend many classical results. This novel technique allows one to tackle the problem of diffusive motion in any geometrical confinement and arbitrary temporal and spatial profile of the magnetic field.

To make a review of a reasonable size, the scope of presented issues has been carefully weighted. The main focus is paid to mathematical aspects of restricted diffusion and their physical interpretations. The slow diffusion and motional narrowing regimes as well as the underlying Gaussian phase approximation and the related notion of apparent diffusion coefficient are investigated in detail. Many other concepts like local gradient approximation, problem of odd moments, cumulant expansion, localization regime or narrow-pulse approximation naturally appear. The use of NMR to probe complex morphologies is also discussed, in particular, the problem of finding surface-to-volume ratio. At the same time, a number of interesting issues could not be included like, for example, diffusion in anisotropic media and related tensorial formalism, or numerous applications in medicine and oil industry. Anomalous diffusion and other "strange" ki- netics, which may occur in nanoporous materials, are not considered [see Kimmich (2002)].

The paper is organized as follows. In the next section, we shall give a short historical overview to emphasize the main contributions to this field. In Section III, a new approach will be established to investigate the macroscopic signal attenuation due to restricted diffusion under arbitrary magnetic field. The moments of the accumulated phase will be found in a compact matrix form involving the Laplace operator eigenbasis in a confining domain. This general approach will be then applied to study spin echoes, the most typical experimental situation. In fact, the rephasing condition, required for echo formation, will make this technique more appropriate for theoretical and numerical use. Different choices of temporal and spatial profiles of magnetic field will be discussed, including linear gradient and parabolic magnetic fields. The explicit calculation of the two matrices $\mathcal{B}$ and $\Lambda$, determining all the moments, will be given for three basic domains (a slab, a cylinder, and a sphere). Section IV is devoted to a detailed study of the slow diffusion regime, where the classical results will be retrieved in a more general form and then critically discussed. In Section V, the leading and correction terms of the moments will be found for the motional narrowing regime. In Section VI, we shall tackle several related topics, from the numerical implementation of this approach to the problem of odd moments. A comparison between theoretical and experimental measurements will be reported. The issues of the localization regime, cumulant expansion and unrestricted diffusion will be discussed. In Conclusion, we shall summarize the essential results and reveal a number of unsolved problems and further perspectives. Appendices collect some interesting but cumbersome calculations outlined in the paper.

\section{A HISTORICAL OVERVIEW}

In this section, we present a short historical overview to emphasize the main contributions and steps in understanding of diffusive NMR phenomena. This can serve as a guide to numerous results which are announced and briefly discussed here, while their more detailed analysis will be given in the following sections.

\section{A. Studying free diffusion by NMR}

The origin of diffusive NMR phenomena can be attributed to Hahn's discovery of spin echoes. Hahn (1950) showed that an application of a $180^{\circ} \mathrm{RF}$ pulse (Fig. 1(a)) leads to refocusing of spin magnetizations, which creates an experimentally measurable signal called "spin echo". Since this macroscopic signal is formed by local contributions of numerous nuclei, it somehow reflects the properties of the whole ensemble. Spin-spin interaction, field inhomogeneities, motion of nuclei and other related effects 
(a)

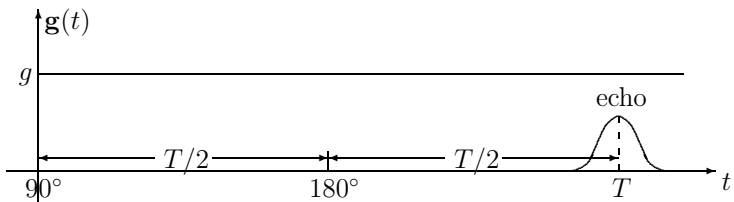

(b)

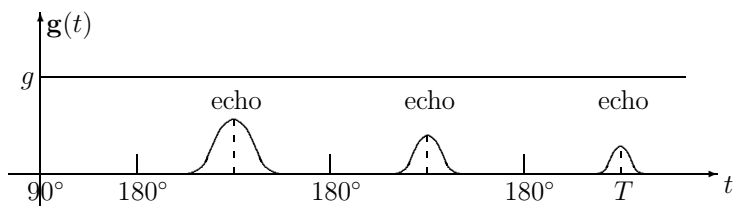

(c)

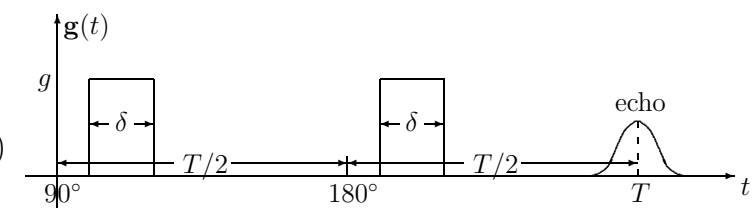

FIG. 1 (a) Formation of a spin echo at time $T$ by $180^{\circ} \mathrm{RF}$ pulse in steady gradient profile (Hahn experiment); (b) Periodic repetition of $180^{\circ} \mathrm{RF}$ pulses creates a train of spin echoes (Carr-Purcell experiment); (c) Pulsed gradient spin echo formation (Stejskal-Tanner experiment).

determine the spin-echo amplitude and can thus be investigated by means of NMR measurements. For instance, Hahn found the attenuation of the macroscopic signal $E$ due to free (or unrestricted) diffusion under steady magnetic field of a linear gradient $g$ and duration $T$ :

$$
E=\exp \left[-D \gamma^{2} g^{2} T^{3} / 12\right],
$$

$D$ being the free self-diffusion coefficient. ${ }^{5}$ On the one hand, the above relation helps to estimate the effect of diffusive motion when studying, for example, spin-spin relaxations. On the other hand, it accesses a direct experimental measurement of the diffusion coefficient by NMR. The Hahn's averaging procedure was improved by Das and Saha (1954).

This fundamental result was further extended by different authors. Carr and Purcell (1954) proposed to repeatedly apply $180^{\circ} \mathrm{RF}$ pulses in order to produce a train of multiple spin echoes as shown in Fig. 1(b). They used a random walk approach to derive the signal attenuation due to free diffusion for $k$-th echo:

$$
E=\exp \left[-D \gamma^{2} g^{2} T^{3} /\left(12 k^{2}\right)\right] .
$$

Again, the effect of diffusive motion can either be eliminated by taking a large number of echoes, or be investigated by studying the dependence on $k$. Its experimental realization was later improved by Meiboom and Gill

5 In the original Hahn result, the prefactor was $1 / 3$ which would correspond to a steady gradient without $180^{\circ} \mathrm{RF}$ pulse. This error was corrected by Carr and Purcell (1954).
(1958) and now is known as Carr-Purcell-Meiboom-Gill (CPMG) technique.

Douglass and McCall (1958) improved Hahn's analysis and gave another experimental verification. From the probabilistic point of view, the accumulated phase $\varphi$ is a random variable whose distribution is determined by the properties of the diffusive motion, the applied magnetic field, and the geometry of confining medium. If there is no geometrical restriction, the Brownian motion can be thought as a sum of independent infinitesimal spatial displacements of a diffusing nucleus. In this case, the central limit theorem (Feller, 1971) ensures a Gaussian distribution of the random phase $\varphi$ in (1):

$$
\mathbb{P}(\varphi)=\left(2 \pi \mathbb{E}\left\{\varphi^{2}\right\}\right)^{-1 / 2} \exp \left(-\frac{\varphi^{2}}{2 \mathbb{E}\left\{\varphi^{2}\right\}}\right)
$$

Like its Fourier transform, the signal is completely determined by the second moment $\mathbb{E}\left\{\varphi^{2}\right\}$ :

$$
E=\exp \left[-\mathbb{E}\left\{\varphi^{2} / 2\right\}\right] .
$$

Douglass and McCall found the second order moment $\mathbb{E}\left\{\varphi^{2}\right\}$ to be equal to $4 D \gamma^{2} g^{2}\left(\tau^{3}-\tau^{2} T+T^{3} / 6\right)$, where $\tau$ is the moment of the $180^{\circ}$ pulse. Taking $\tau=T / 2$, one gets $\mathbb{E}\left\{\varphi^{2} / 2\right\}=D \gamma^{2} g^{2} T^{3} / 12$ in agreement with (3).

Stejskal and Tanner (1965) proposed to replace a steady gradient by a pulsed gradient to facilitate the measurement and to enlarge the ranges of applicability. A typical pulsed gradient profile is shown in Fig. 1(c). If there is no gradient during the RF pulses, their amplitude need not be particularly large; in turn, no gradient at the echo time $T$ makes the determination of the echo amplitude more precise. In their paper, Stejskal and Tanner found the signal attenuation due to free diffusion for arbitrary temporal profile of a linear magnetic field gradient $\mathbf{g}(t)$ :

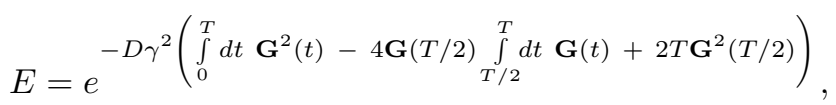

where $\mathbf{G}(t)=\int_{0}^{t} d t^{\prime} \mathbf{g}\left(t^{\prime}\right)$ and the $180^{\circ} \mathrm{RF}$ pulse is applied at time $T / 2$. This general relation and its derivatives for particular temporal profiles are widely used until nowadays for theoretical and experimental purposes. For instance, the signal attenuation for a pulsed gradient shown in Fig. 1(c) was found to be

$$
E=\exp \left[-D \gamma^{2} g^{2} \delta^{2}(T / 2-\delta / 3)\right]
$$

One retrieves the Hahn's result by taking $\delta=T / 2$. We shall thoroughly discuss these results in the following sections.

\section{B. Bloch-Torrey equation}

An alternative description of diffusive NMR phenomena was proposed by Torrey (1956). To account for the 
diffusive motion, he modified the Bloch equation (Bloch, 1946) for the spin magnetization $\mathfrak{m}(\mathbf{r}, t)$ :

$$
\left(\frac{\partial}{\partial t}-D \Delta+i \gamma \mathbf{g} \cdot \mathbf{r}\right) \mathfrak{m}(\mathbf{r}, t)=0,
$$

where $\Delta$ is the Laplace operator. Looking for a solution of the one-dimensional equation in the form $\mathfrak{m}(x, t)=$ $A(t) e^{-i \gamma g x t}$, Torrey retrieved the relations (3), (4) in a simpler way.

An introduction of appropriate boundary conditions makes the equation (9) suitable to describe the restricted diffusion in a confining domain $\Omega$. In general, the Fourier (or mixed, or relaxing) boundary condition is used to take into account the surface relaxation or permeability of the boundary $\partial \Omega$ :

$$
D \frac{\partial}{\partial n} \mathfrak{m}(\mathbf{r}, t)=K \mathfrak{m}(\mathbf{r}, t)
$$

where $K$ is the relaxation rate (or interface permeability), and $\partial / \partial n$ is the normal derivative directed towards the bulk. The above relation is nothing else than a conservation law: at each boundary point, the fluxes of magnetization from the bulk and through the boundary are equal. The role of the Fourier boundary condition for the Laplacian transport phenomena was thoroughly discussed (Filoche and Sapoval, 1999; Grebenkov et al., 2003, 2006c; Sapoval, 1994). If the interface is impermeable for spins and does not contain magnetic impurities which could lead to a surface relaxation (i.e., $K=0$ ), the above condition is reduced to the Neumann (or reflecting) boundary condition on the boundary $\partial \Omega$ :

$$
\frac{\partial}{\partial n} \mathfrak{m}(\mathbf{r}, t)=0
$$

Once the magnetization $\mathfrak{m}(\mathbf{r}, t)$ is found by solving the Bloch-Torrey equation with a given initial condition $\rho(\mathbf{r})$, the signal amplitude $E$ at time $T$ is obtained by integration of $\mathfrak{m}(\mathbf{r}, T)$ over the whole confining domain $\Omega$ :

$$
E=\int_{\Omega} d \mathbf{r} \mathfrak{m}(\mathbf{r}, T) .
$$

The Neumann boundary condition (11) was used by Kaplan (1959) to describe electron spin resonance in metals. After Wayne and Cotts (1966) who solved numerically the Bloch-Torrey equation in a laminar system with the reflecting boundary condition, this approach became quite common in numerical analysis of diffusive NMR phenomena.

\section{Effect of a geometrical restriction}

Relations (3) - (7) were derived by different methods that commonly used the assumption of unrestricted diffusion: the nuclei diffuse freely in an infinite reservoir. Woessner used the spin-echo technique to experimentally demonstrate the effect of a geometrical restriction. The signal attenuation was measured for water molecules in a geological core and in aqueous suspensions of silica spheres (Woessner, 1960, 1961, 1963). Although $\ln E$ was still proportional to $g^{2}$, the ratio $D^{\prime}=-\ln E /\left(\gamma^{2} g^{2} T^{3} / 12\right)$ was not equal to the free diffusion coefficient $D$ of water. Woessner called this ratio a "spin-echo diffusion coefficient" which is now known as "effective or apparent diffusion coefficient". The decrease of $D^{\prime}$ with $T$ was attributed to the growing proportion $\sqrt{D T} / L$ of molecules whose motion was restricted by boundaries of the reservoir of size $L$.

\section{Narrow-pulse approximation}

The first theoretical treatment of restricted diffusion was proposed by Stejskal (1965) and Tanner and Stejskal (1968). Stejskal modified the Bloch-Torrey equation (9) to include the case of anisotropic, restricted diffusion and flow. Then, the restricted diffusion was studied by the "pulsed (field) gradient spin-echo" (PGSE) method. If the duration $\delta$ of gradient pulses is small compared to the characteristic diffusion time $L^{2} / D$, the macroscopic signal can be related to the Fourier transform of the Green function in the confining medium $\Omega$. Indeed, the first gradient pulse encodes the position $\mathbf{r}$ of a nucleus at time $t=0$ by factor $e^{i \gamma \delta \mathbf{g} \cdot \mathbf{r}}$, while the second gradient pulse labels the position $\mathbf{r}^{\prime}$ of the same nucleus at time $t=T$ by factor $e^{-i \gamma \delta \mathbf{g} \cdot \mathbf{r}^{\prime}}$ (the sign is changed by the $180^{\circ} \mathrm{RF}$ pulse). The probability density to diffuse from $\mathbf{r}$ to $\mathbf{r}^{\prime}$ during time $T$ is known as propagator or Green function $G_{T}\left(\mathbf{r}, \mathbf{r}^{\prime}\right)$ (see subsection III.A). The average over initial and final positions leads to the macroscopic signal:

$$
E=\frac{1}{V} \int_{\Omega} d \mathbf{r} \int_{\Omega} d \mathbf{r}^{\prime} G_{T}\left(\mathbf{r}, \mathbf{r}^{\prime}\right) e^{i \gamma \delta \mathbf{g} \cdot\left(\mathbf{r}-\mathbf{r}^{\prime}\right)},
$$

$V$ being the volume of the domain $\Omega$. The crucial simplification of this approach, known now as "narrow-pulse or short-gradient-pulse approximation" (NPA), is an explicit manifestation of the confining geometry via the Green function. The PGSE method allows one a direct experimental measurement of the Fourier transform of the Green function, and gives thus a simple way to probe a complex morphology by NMR. This technique, also called " $q$-space imaging method", was widely used for both theoretical and experimental studies (Callaghan, 1991). For instance, the effect of diffusion diffraction was predicted and discovered by Callaghan, Coy, MacGowan, Packer and Zelaya (1991) and further extended by Callaghan, Coy, Halpin, MacGowan, Packer and Zelaya (1992) and by Coy and Callaghan (1994). An analogy with X-ray diffraction and electron or neutron scattering was also discussed by Cotts (1991). A similar behavior for a cylindrical pore was reported by Söderman 
and Jönsson (1995) and by Gibbs (1997). Balinov, Söderman and Ravey (1994) observed the diffraction-like effects in highly concentrated water-oil emulsion. Their manifestation in the case of porous media was discussed by Bergman and Dunn (1994) and by Sen, Hürlimann and de Swiet (1995). The effect of surface relaxation was considered by Mitra and Sen (1992). In particular, the influence of surface relaxation on the apparent diffusion coefficient in the long time limit was studied by Sen, Schwartz, Mitra and Halperin (1994). The relation between pulsed gradient NMR measurements of restricted diffusion and the return-to-the-origin probability was pointed out by Mitra, Latour, Kleinberg, and Sotak (1995b) and by Schwartz, Hürlimann, Dunn, Mitra, and Bergman (1997). King, Houseman, Roussel, van Bruggen, Williams and Gadian (1994) used the $q$ space concept for imaging of the brain. An original approach to describe the diffusion diffraction was developed by Stepišnik (1998). He proposed to express the nonuniform spin phase distribution in a pore as a series of waves with wave vectors characterizing the geometry and boundaries of confinement.

The validity of the approximate relation (13) and applicability of this concept in different NMR contexts were extensively investigated by different authors. Blees (1994) numerically solved a modified Bloch-Torrey equation by finite difference method in order to quantify the effect of finite duration of gradient pulses. For the same purpose, Linse and Söderman (1995) performed Brownian dynamic simulations for molecules entrapped in planar, cylindrical and spherical geometries. Mitra and Halperin (1995) studied how the finite duration of gradient pulses makes isolated pore appear smaller than their actual size. Wang, Caprihan and Fukushima (1995) proposed a criterion of applicability of the NPA by considering restricted diffusion in simple domains (slab, cylinder and sphere). Monte Carlo simulations were also implemented by Duh, Mohorič and Stepišnik (2001) to show a strong deviation from approximate theoretical results in the case of intermediate and long sequences. Mair, Sen, Hürlimann, Patz, Cory and Walsworth (2002) showed a breakdown of the narrow-pulse approximation by analyzing the restricted diffusion of xenon in model porous media (random packs of mono-sized glass beads). Malmborg, Topgaard and Söderman (2004) studied highly concentrated emulsions to show how the duration of the gradient pulse influences NMR diffusion experiments. We shall discuss the narrow-pulse approximation in subsection VI.E.

\section{E. Restricted diffusion in simple domains}

Robertson (1966) applied a quantum mechanics operator formalism to study restricted diffusion in a slab geometry (between two parallel planes). The magnetization $\mathfrak{m}(\mathbf{r}, t)$, satisfying the Bloch-Torrey equation (9) with Neumann boundary condition (11), was expanded over eigenfunctions $u_{m}(\mathbf{r})$ of the Laplace operator on an interval

$$
\mathfrak{m}(\mathbf{r}, t)=\sum_{m} c_{m}(t) u_{m}(\mathbf{r})
$$

An infinite-dimension system of linear differential equations was obtained for unknown coefficients $c_{m}(t)$. A further analysis of the density matrix $c_{m}(t) c_{m^{\prime}}^{*}(t)$ led to an approximate relation

$$
\begin{aligned}
-\ln E \simeq & q^{2} \sum_{m=0}^{\infty} \frac{8}{\pi^{4}(2 m+1)^{4}}\left(\frac{1}{\pi^{2}(2 m+1)^{2} p}-\right. \\
& \left.-\frac{3-4 e^{-\pi^{2}(2 m+1)^{2} p / 2}+e^{-\pi^{2}(2 m+1)^{2} p}}{\pi^{4}(2 m+1)^{4} p^{2}}\right),
\end{aligned}
$$

where $q=\gamma g L T$ and $p=D T / L^{2}$. For small time $T(p \ll$ $\left.1 / \pi^{2}\right)$, a series expansion of the exponential functions in (15) leads to the Hahn's relation (3). For long enough $T\left(p \gg 2 / \pi^{2}\right)$, Robertson found a new behavior of the signal attenuation due to restricted diffusion in a slab geometry, which is now called "motionally averaging or motional narrowing regime":

$$
E \simeq \exp \left[-\gamma^{2} g^{2} L^{4} T /(120 D)\right]
$$

Although $\ln E$ is still proportional to $g^{2}$, the dependence on the gradient duration $T$, the diffusion coefficient $D$ and the slab width $L$ is drastically different in comparison with Eq. (3). For instance, the nuclei with higher $D$ diffuse more rapidly, but the related signal attenuation is, contrarily to intuition, smaller. A sharp dependence on the size of the confining domain appears here as a characteristic feature of restricted diffusion. This behavior was experimentally observed in a laminar system in the same year by Wayne and Cotts (1966). We shall discuss the motional narrowing regime in detail in Section $\mathrm{V}$.

The Robertson's work had a deep impact on a posterior development of this field. A high sensitivity to the geometrical confinement stimulated the interest to restricted diffusion as a way to probe confining morphologies of porous materials and biological tissues by NMR. A number of far-reaching extensions were made. So, Stepišnik (1981, 1985) developed the density matrix calculation for a much more general case including, e.g., strong dipolar coupling. In particular, the NMR spin-echo attenuation in the magnetic field gradient was related to the velocity autocorrelation function (Stepišnik, 1993).

The eigenfunction expansion of the magnetization became a usual tool to study restricted diffusion. For instance, Brownstein and Tarr (1979) used it to explain multiexponential relaxation for water in biological cells. Song (2000) reported an experimental demonstration of the excitation and detection of a wide range of eigenmodes in porous media by exploring the inhomogeneous internal magnetic field in the pore space. This interesting technique was applied to characterize multiple length scales in rocks by Song, Ryu and Sen (2000). 
Finally, Robertson's operator formalism was a prototype for efficient numerical techniques like multiple propagator or step-wise gradient approach (see subsection II.J). The multiple correlation function approach (Section III) has been conceptually issued from Robertson's work.

\section{F. Gaussian phase approximation}

Neuman (1974) retrieved and extended the Robertson's results by considering the accumulation of phase shifts during diffusive motion. In the limit of short diffusion times, only a small fraction of nuclei can "feel" the presence of reflecting boundaries, and their contribution to the accumulated phase can be neglected. One thus retrieves a Gaussian distribution (5). In the opposite limit of very long diffusion times, each spin explores the bulk several times during a fixed time interval. At this time scale, the spatial displacements of a spin can be seen as independent "jumps" at randomly chosen bulk points, and the central limit theorem leads again to the relation (5). Neuman assumed that a Gaussian distribution was valid even for the intermediate case. This assumption, known now as "Gaussian phase approximation" (GPA), reduces a difficult problem of resolving the Bloch-Torrey equation in confining medium to the calculation of the second moment $\mathbb{E}\left\{\varphi^{2}\right\}$ which is in general much simpler. In particular, Neuman carried out an analytical calculation of restricted diffusion in a slab, a cylinder and a sphere. For these "basic" domains, the Green function and the eigenfunctions of the Laplace operator are known explicitly (see Table I).

After Neuman's work, the Gaussian phase approximation was repeatedly used by many authors. Murday and Cotts (1968) observed experimentally the restricted diffusion of liquid lithium in spherical droplets and confirmed GPA. To explain transverse relaxation processes in porous sedimentary rocks, Kleinberg and Horsfield (1990) modeled pores as spherical cavities and then applied Neuman's results. Hayden, Archibald, Gilbert and Lei (2004) used them to fit the experimental measurements for the restricted diffusion of hyperpolarized helium-3 in a cylindrical pore. Kuchel, Lennon and Durrant (1996) extended Neuman's relation for a sphere to account for surface and bulk relaxation. A theory of the macroscopic signal formation in presence of structurespecific magnetic field inhomogeneities, developed by Sukstanskii and Yablonskiy (2003, 2004), was based on the Gaussian phase approximation. The accuracy of the GPA was numerically investigated by different authors. For example, Balinov, Jönsson, Linse, and Söderman (1994) used Brownian dynamics to simulate the motion of spins confined to spheres and between planes.

The Gaussian phase approximation and Neuman's results will be discussed in detail in Section V.

\section{G. Non-Gaussian behavior}

Neuman's relations were in such good agreement with numerical and experimental observations that the Gaussian phase approximation was believed to be correct for any set of physical parameters. In particular, Stepišnik (1999) gave a general phenomenological estimate for the gradient intensity $(g \leq 40 \mathrm{~T} / \mathrm{m}$ for gases and $g \leq$ $100 \mathrm{~T} / \mathrm{m}$ for liquids) under which the Gaussian phase approximation was supposed to be correct.

The discovery of a non-Gaussian behavior was like a bolt from the blue. Stoller, Happer and Dyson (1991) studied the spectral properties of the Bloch-Torrey equation on an interval that corresponds to restricted diffusion in a slab geometry. For a large gradient intensity $g$, they demonstrated a non-Gaussian decrease of the macroscopic signal as a function of $g$. The physical consequences of this new and unexpected behavior was discussed by de Swiet and Sen (1994). This socalled "localization regime" was experimentally observed for the first time by Hürlimann, Helmer, de Swiet, Sen and Sotak (1995). They studied the restricted diffusion of water molecules between two parallel plates at distance $0.16 \mathrm{~mm}$. A drastic deviation from the Gaussian $g^{2}$ dependence of the $\ln E$ was observed at gradient intensity higher that $15 \mathrm{mT} / \mathrm{m}$ (see subsection VI.F and Fig. 7). This corresponds to the case when the gradient length $(D / \gamma g)^{1 / 3}$, over which the spins dephase by $2 \pi$, becomes shorter than both the diffusion length $\sqrt{D T}$ and the plate separation $L$. The manifestation of localization regime for a CPMG sequence was analyzed by Sen, André, Axelrod (1999) and by Zhang and Hirasaki (2003). We shall continue this discussion in subsection VI.F.

This important discovery forced physicists to revise a common belief in the Gaussian phase approximation and stimulated a number of important researches in this field. The knowledge of the fourth and higher order moments of the accumulated phase appeared to be mandatory for a better understanding of diffusive NMR phenomena. This fundamental problem was studied by Bergman and Dunn (1995) who calculated the fourth moment in the case of a periodic porous medium. Some of their interesting ideas were employed by the author to develop a systematic technique to compute the multiple correlation functions of the reflected Brownian motion (see section III).

\section{H. Studying porous materials}

In the nineties, a series of papers ${ }^{6}$ was devoted to the study of restricted diffusion in porous media in the short time limit. Starting from Woessner's qualitative

\footnotetext{
6 de Swiet and Sen (1994); Helmer, Hürlimann, de Swiet, Sen and Sotak (1995); Latour, Mitra, Kleinberg and Sotak (1993); Mitra, Sen, Schwartz and Le Doussal (1992); Mitra, Sen, and Schwartz (1993).
} 
arguments, Mitra et al. developed and experimentally checked a quantitative theory to account for the contribution of nuclei whose motion was restricted by boundaries of the confining medium. The effective diffusion coefficient was found at short times:

$$
D_{a p p} \simeq D\left(1-\alpha \sqrt{D T} \frac{S}{V}\right)
$$

where $S / V$ is the surface-area-to-pore-volume ratio of the confining medium. The numerical prefactor $\alpha$ was analytically computed for steady and pulsed gradient profiles shown in Fig. 1(a),(c) (see subsection IV.E). NMR techniques appeared thus as promising experimental tools to measure the surface-to-volume ratio, the intrinsic characteristic which plays an important role in oil industry and medical diagnostics. So, the notion of effective time-dependent diffusion coefficient was applied by Helmer, Dardzinski and Sotak (1995b) to investigate in vivo systems and by Mair, Cory, Peled, Tseng, Patz, and Walsworth (1998), by Mair, Wong, Hoffmann, Hürlimann, Patz, Schwartz and Walsworth (1999) and by Mair, Rosen, Wang, Cory and Walsworth (2002b) to the case of granular materials. Probing short length scales in a static gradient with the CPMG sequence was proposed by Zielinski and Hürlimann (2005). We shall discuss the result (17) in detail in section IV.

\section{Nonlinear magnetic fields}

Although the investigation of restricted diffusion in a linear magnetic field gradient counts hundreds of references, there were only few theoretical works devoted to nonlinear magnetic fields. This situation may appear a bit surprising since hardware imperfections unavoidably lead to smaller or bigger deviations from a linear gradient. Moreover, the difference in magnetic susceptibilities of porous and bulk media creates a structuredependent distribution of local inhomogeneities of the magnetic field. These fields were difficult to control experimentally and to investigate theoretically by classical approaches.

The first theoretical study of the effect of nonlinear magnetic fields on restricted diffusion was proposed by Tarczon and Halperin (1985). In the case of onedimensional diffusion restricted on an interval, an arbitrary spatial profile of the magnetic field was represented by a Fourier series. Its coefficients were shown to determine the second moment of the accumulated phase, and the Gaussian phase approximation gave the macroscopic signal. In particular, Tarczon and Halperin proposed an approximate relation in the short time limit:

$$
E \approx \exp \left[-D \gamma^{2} g_{e f f}^{2} T^{3} / 12\right]
$$

where $g_{\text {eff }}^{2}=<(\nabla B(\mathbf{r}))^{2}>$ is the spatial average of the squared gradient of the magnetic field. Tarczon and Halperin argued that the signal attenuation in a nonlinear magnetic field $B(\mathbf{r})$ could be characterized by an effective gradient $g_{\text {eff }}$. This result was applied by Hürlimann (1998) to take into account susceptibilityinduced gradients in porous media. In the simple case of a linear gradient, $B(\mathbf{r})=\mathbf{g} \cdot \mathbf{r}$, one could get $g_{\text {eff }}^{2}=g^{2}$ and retrieve the Hahn's relation (3). Moreover, Tarczon and Halperin suggested a more accurate result which is now known as "local gradient approximation":

$$
E \simeq \frac{1}{V} \int_{\Omega} d \mathbf{r} \exp \left[-\frac{D \gamma^{2} T^{3}}{12}(\nabla B(\mathbf{r}))^{2}\right] .
$$

We shall discuss this result in section IV.

The other theoretical treatments of nonlinear magnetic fields in the whole space (i.e., unrestricted diffusion) have to be mentioned. The first one was given by Majumdar and Gore (1988), who proposed to model the spatial distribution of susceptibility-induced magnetic field inhomogeneities as discrete random fields. The effective diffusion coefficient (or relaxation rate) was found to be proportional to the variance of the distribution of the magnetic field gradients. The case of continuous Gaussian random fields was considered by Mitra and Le Doussal (1991). In the long time limit, they found an exponential decrease of the macroscopic signal in time $T$ with a power law correction. The problem of large fluctuations in finite-size systems was discussed.

Le Doussal and Sen (1992) derived an exact solution of the Bloch-Torrey equation in the whole space for quadratic (or parabolic) magnetic field profile, $B(z)=$ $g_{0}+g_{1} z+g_{2} z^{2}$. In the short time limit, the signal attenuation was similar to that of the "effective" linear gradient, in agreement with the approximate relation (18). In the long time limit, however, Le Doussal and Sen found that $\ln E$ was proportional to $T$ instead of the Hahn's $T^{3}$-dependence. A natural length scale $\left(8 D / \gamma g_{2}\right)^{1 / 4}$ was shown to govern the problem. Parabolic magnetic fields were studied experimentally by Bendel (1990).

Finally, Zielinski and Sen (2000) reported a numerical solution of the one-dimensional Bloch-Torrey equation with parabolic and cosine spatial profiles of the magnetic field. The evolution of the signal was argued to be largely determined at all times solely by the two "moments" of the magnetic field, $\left\langle(\nabla B)^{2}>\right.$ and $<\left(\int d x B(x)\right)^{2}>$, and not by the details of its local spatial distribution. They also showed that the local gradient approximation holds only in the short time limit and is invalid for longer times.

\section{J. Matrix formalisms}

A numerical study of restricted diffusion would deserve another review. To calculate the attenuation of the macroscopic signal, different authors performed either a numerical resolution of the Bloch-Torrey equation, or Monte Carlo simulations of the reflected Brownian motion. In the former case, the bulk of a confining domain 
was discretized by a regular lattice or a more complicated mesh, so that a discrete version of the Bloch-Torrey equation could be numerically solved by finite difference or finite element method. The distribution of the spin magnetization $\mathfrak{m}(\mathbf{r}, t)$ over lattice sites was sequentially calculated with a small time step $\tau$. The macroscopic signal was then given as spatial average of $\mathfrak{m}(\mathbf{r}, t)$. In contrast, Monte Carlo techniques were used to simulate diffusive motion and compute accumulated phase of an individual spin. Brownian trajectories were usually modeled as a sequence of independent random "jumps" in the bulk with reflections on the boundary. The jump distance in each spatial direction was normally distributed with dispersion $\sqrt{2 D \tau}$. Once the accumulated phase distribution $\mathbb{P}(\varphi)$ was approximately found by launching a large number of random walkers, the signal could be deduced as expectation of $e^{i \varphi}$.

Although efficient for some purposes, these techniques present inconveniences and limitations. For instance, the statistical error of Monte Carlo simulations decreases slowly with the number of random walkers; the study of intensive gradients requires a very fine resolution (or discretization) of the Bloch-Torrey equation; long time analysis is in general difficult by both techniques, etc. To overcome these problems, Caprihan, Wang and Fukushima (1996) proposed an original numerical approach. The idea was to approximate a given temporal gradient profile $\mathbf{g}(t)$ by a large sum of equidistant very narrow gradient pulses at times $k \tau, \tau$ being a short time interval between two successive pulses. These gradient pulses encode the successive positions $\mathbf{r}_{k}=\mathbf{r}(k \tau)$ of a diffusing nucleus by factors $e^{i \gamma \delta \mathbf{g}_{k} \cdot \mathbf{r}_{k}}$, where $\mathbf{g}_{k}=\mathbf{g}(k \tau)$. The random displacements of nuclei during the time interval $\tau$ is determined by the Green function $G_{\tau}\left(\mathbf{r}_{k}, \mathbf{r}_{k+1}\right)$. To calculate the average over all positions $\mathbf{r}_{k}$, Caprihan et al. used the expansion of the Green function over the Laplace operator eigenfunctions (see Appendix E for details). A general but cumbersome expression for the macroscopic signal was derived. As example, they considered the case of restricted diffusion between two parallel planes.

The original approach by Caprihan et al. was reformulated by Callaghan (1997) in a simple and elegant matrix form. He showed that the macroscopic signal can be written within a matrix formalism involving the eigenbasis of the Laplace operator (see Appendix E for details). Practically, the numerical problem of finding the signal attenuation under arbitrary temporal gradient profile was reduced to symbolic manipulation with two matrices which depend on several physical parameters. Callaghan illustrated the efficiency of this "multiple propagator approach" by considering restricted diffusion between two parallel planes. In a further work, Callaghan and Codd (1998) and Codd and Callaghan (1999) studied restricted diffusion in a cylinder and a sphere and discussed the role of surface relaxation. Price, Stilbs and Söderman (2003) used this technique to check the validity of the narrow-pulse approximation. Grebenkov (2006) developed a spectral analysis of the underlying matrices to show multiexponential signal attenuation for CPMG sequences.

The Callaghan's matrix formalism was reformulated in terms of random walks by Sukstanskii and Yablonskiy (2002). Using the modified approach, they obtained a number of interesting results, in particular, they found a transition between oscillatory and monotonic behaviors of the free induction decay signal as a function of time. The accuracy of the Gaussian phase approximation was also discussed.

Finally, Barzykin $(1998,1999)$ proposed an equivalent matrix formalism by considering a step-wise approximation of the temporal gradient profile. In his approach, the two matrices determining the macroscopic signal depend solely on the confining geometry. These matrices have thus to be calculated only once for a chosen confining medium (e.g., a sphere), after that the computation of the signal is straightforward and rapid for any set of physical parameters. This is an important improvement of the above matrix techniques and a crucial simplification for numerical analysis.

The different matrix approaches outlined above were mainly intended for numerical computation of the macroscopic signal. In the next section, an extension of the Barzykin's technique will be introduced to tackle theoretically the problem of restricted diffusion in arbitrary magnetic field. This "multiple correlation function approach" will be applied throughout the paper to retrieve, extend and critically discuss numerous results shortly presented in the above overview.

\section{MULTIPLE CORRELATION FUNCTION APPROACH}

In this section, the moments of a random phase accumulated by a diffusing spin are found in a matrix form involving the Laplace operator eigenbasis in a confining domain. Spatial inhomogeneities and time dependence of the magnetic field enter as functionals and weight factors to the multiple correlation functions. After a formulation for a general case in subsection III.A, this MCF approach is applied to spin echoes in subsection III.B. Different choices of temporal and spatial profiles of the magnetic field are considered in subsections III.C and III.D respectively. In subsection III.E, the analytical results are derived for the restricted diffusion in three simple domains (slab, cylinder and sphere).

\section{A. General case}

As a first step, we reformulate the general physical description (1), (2) of diffusive NMR phenomena in such a way that will allow us their further theoretical and numerical study. For this purpose, the exponential function 
in (2) is expanded in a power series

$$
E=\sum_{n=0}^{\infty} \frac{i^{n}}{n !} \mathbb{E}\left\{\varphi^{n}\right\}
$$

where the moments of the random variable $\varphi$ are

$\mathbb{E}\left\{\varphi^{n}\right\}=\gamma^{n} \mathbb{E}\left\{\int_{0}^{T} d t_{1} \ldots \int_{0}^{T} d t_{n} B_{t_{1}}\left(\mathbf{r}\left(t_{1}\right)\right) \ldots B_{t_{n}}\left(\mathbf{r}\left(t_{n}\right)\right)\right\}$.

The multiple integral of the product of "identical" functions can be written as ordered time average

$\mathbb{E}\left\{\varphi^{n}\right\}=n ! \gamma^{n} \int_{0}^{T} d t_{1} \ldots \int_{t_{n-1}}^{T} d t_{n} \mathbb{E}\left\{B_{t_{1}}\left(\mathbf{r}\left(t_{1}\right)\right) \ldots B_{t_{n}}\left(\mathbf{r}\left(t_{n}\right)\right)\right\}$,

where the time moments $t_{1}, \ldots, t_{n}$ are now in ascending order. The multiple correlation function $\mathbb{E}\left\{B_{t_{1}}\left(\mathbf{r}\left(t_{1}\right)\right) \ldots B_{t_{n}}\left(\mathbf{r}\left(t_{n}\right)\right)\right\}$ can be calculated according to its probabilistic meaning:

- the starting position $\mathbf{r}_{0}$ of the reflected Brownian motion is chosen with a given initial density $\rho\left(\mathbf{r}_{0}\right)$;

- the probability density for arriving from this point to a random position $\mathbf{r}_{1}=\mathbf{r}\left(t_{1}\right)$ at time $t_{1}$ is given by the Green function $G_{t_{1}}\left(\mathbf{r}_{0}, \mathbf{r}_{1}\right)$ of the diffusion operator in domain $\Omega$

$$
\left(\frac{\partial}{\partial t}-D \Delta\right) G_{t}\left(\mathbf{r}, \mathbf{r}^{\prime}\right)=0
$$

with the appropriate Fourier or Neumann boundary condition, and $G_{t=0}\left(\mathbf{r}, \mathbf{r}^{\prime}\right)=\delta\left(\mathbf{r}-\mathbf{r}^{\prime}\right), \delta$ being the Dirac distribution (delta-function);

- the subsequent positions $\mathbf{r}_{2}=\mathbf{r}\left(t_{2}\right), \ldots, \mathbf{r}_{n}=$ $\mathbf{r}\left(t_{n}\right)$ are also distributed according to the corresponding probability densities $G_{t_{2}-t_{1}}\left(\mathbf{r}_{1}, \mathbf{r}_{2}\right), \ldots$, $G_{t_{n}-t_{n-1}}\left(\mathbf{r}_{n-1}, \mathbf{r}_{n}\right)$.

Since the reflected Brownian motion is a Markovian process (without memory), the multiple correlation function is

$$
\begin{aligned}
& \mathbb{E}\left\{B_{t_{1}}\left(\mathbf{r}\left(t_{1}\right)\right) \ldots B_{t_{n}}\left(\mathbf{r}\left(t_{n}\right)\right)\right\}=\int_{\Omega} d \mathbf{r}_{0} \ldots \int_{\Omega} d \mathbf{r}_{n} \rho\left(\mathbf{r}_{0}\right) \times \\
& \times G_{t_{1}}\left(\mathbf{r}_{0}, \mathbf{r}_{1}\right) B_{t_{1}}\left(\mathbf{r}_{1}\right) G_{t_{2}-t_{1}}\left(\mathbf{r}_{1}, \mathbf{r}_{2}\right) \ldots B_{t_{n}}\left(\mathbf{r}_{n}\right) .
\end{aligned}
$$

A similar relation was used by Bergman and Dunn (1995) to describe the signal attenuation in periodic porous media.

To proceed, one can use the spectral decomposition of the Green function over the eigenfunctions $u_{m}(\mathbf{r})$ of the Laplace operator (Arfken and Weber, 2001)

$$
G_{t}\left(\mathbf{r}, \mathbf{r}^{\prime}\right)=\sum_{m=0}^{\infty} u_{m}(\mathbf{r}) u_{m}^{*}\left(\mathbf{r}^{\prime}\right) \exp \left[-D t \lambda_{m} / L^{2}\right],
$$

where $L$ is a characteristic dimension of the confining domain $\Omega$ (e.g., its diameter), and the asterisk denotes the complex conjugate. The eigenvalues $\lambda_{m}$ are defined to be dimensionless:

$$
\Delta u_{m}(\mathbf{r})=-\left(\frac{\lambda_{m}}{L^{2}}\right) u_{m}(\mathbf{r})
$$

and the Neumann or Fourier boundary condition is imposed according to the physical properties of the interface. The eigenfunctions $u_{m}(\mathbf{r})$ are orthonormal in the space $L^{2}(\Omega)$ of measurable and square integrable functions:

$$
\int_{\Omega} d \mathbf{r} u_{m}(\mathbf{r}) u_{m^{\prime}}^{*}(\mathbf{r})=\delta_{m, m^{\prime}},
$$

where $\delta_{m, m^{\prime}}$ is the Kronecker symbol. For theoretical analysis, it is convenient to introduce the dimensionless diffusion coefficient

$$
p=D T / L^{2} .
$$

The substitution of the spectral decomposition (25) in Eq. (24) for each Green function leads to

$$
\begin{aligned}
\mathbb{E}\{ & \left.B_{t_{1}}\left(\mathbf{r}\left(t_{1}\right)\right) \ldots B_{t_{n}}\left(\mathbf{r}\left(t_{n}\right)\right)\right\}=\sum_{m_{1}=0}^{\infty} \ldots \sum_{m_{n}=0}^{\infty} U_{m_{1}} e^{-p \lambda_{m_{1}} t_{1} / T} \\
& \times \mathcal{B}_{m_{1}, m_{2}}\left(t_{1}\right) e^{-p \lambda_{m_{2}}\left(t_{2}-t_{1}\right) / T} \mathcal{B}_{m_{2}, m_{3}}\left(t_{2}\right) \ldots \hat{U}_{m_{n}}\left(t_{n}\right),
\end{aligned}
$$

where the infinite-dimension matrix $\mathcal{B}$ and vectors $U$ and $\hat{U}(t)$ are defined as ${ }^{7}$

$$
\begin{aligned}
\mathcal{B}_{m, m^{\prime}}(t) & =\int_{\Omega} d \mathbf{r} u_{m}^{*}(\mathbf{r}) B_{t}(\mathbf{r}) u_{m^{\prime}}(\mathbf{r}) \\
U_{m} & =V^{1 / 2} \int_{\Omega} d \mathbf{r} u_{m}(\mathbf{r}) \rho(\mathbf{r}) \\
\hat{U}_{m}(t) & =V^{-1 / 2} \int_{\Omega} d \mathbf{r} u_{m}^{*}(\mathbf{r}) B_{t}(\mathbf{r})
\end{aligned}
$$

and $V$ is the volume of the domain $\Omega$. The summation over indices $m_{1}, \ldots, m_{n}$ can be thought of as a matrix product that leads to a compact representation of a scalar product for the multiple correlation function

$$
\begin{aligned}
& \mathbb{E}\left\{B_{t_{1}}\left(\mathbf{r}\left(t_{1}\right)\right) \ldots B_{t_{n}}\left(\mathbf{r}\left(t_{n}\right)\right)\right\}= \\
& U \cdot\left(\left[\prod_{j=1}^{n-1} e^{-p \Lambda t_{j} / T} \mathcal{B}\left(t_{j}\right) e^{p \Lambda t_{j} / T}\right] e^{-p \Lambda t_{n} / T} \hat{U}\left(t_{n}\right)\right),
\end{aligned}
$$

7 The initial density $\rho(\mathbf{r})$ might be alternatively set to the inverse of the domain volume $V$ provided that eigenfunctions $u_{m}(\mathbf{r})$ are appropriately normalized [see, for example, Barzykin (1999)]. In practice, however, such a tricky normalization is quite difficult, so that we prefer to use the classical expansion (25) for the Green function. 
with the diagonal infinite-dimension matrix $\Lambda$ :

$$
\Lambda_{m, m^{\prime}}=\delta_{m, m^{\prime}} \lambda_{m}
$$

The ordered time average of the above correlation function gives the moment $\mathbb{E}\left\{\varphi^{n}\right\}$ of the random phase $\varphi$ and determines the $n$-th order contribution to the signal expansion (20). An unbounded increase of the eigenvalues $\lambda_{m}$ with $m$ ensures a rapid convergence in the matrix product (33) and makes possible a truncation of the matrices $\mathcal{B}$ and $\Lambda$ to a limited dimension for numerical analysis.

At first thought, one may wonder what is the interest of such a formal approach? The compact and transparent physical description by Eqs. (1), (2) is "reduced" to a cumbersome mathematical formalism involving the multiple time integration of the product of timedependent infinite-dimension matrices. Even for numerical simulations, modeling the reflected Brownian motion might seem to be more simple and efficient. However, this thought is misleading: the deceptive simplicity of Eqs. (1), (2) relies in the fact that the diversity of diffusive NMR phenomena is "hidden" in a very complex behavior of the reflected Brownian motion. The stochastic character of diffusive motion is entangled with particular properties of the confining domain and applied magnetic field. The complexity of these phenomena made them attractive for physicists since the Hahn's seminal paper in 1950 till our days. The above matrix formalism is in fact a general mathematical basis to study the diffusive motion in any confining geometry under arbitrary magnetic field. In this formal way, the physical problem of finding the macroscopic signal of diffusing spins is entirely reduced to the analysis of the Laplace operator eigenmodes, and thus solved, as a physical problem. In what follows, we shall show how this mathematical basis can be applied for a theoretical analysis in many cases of particular interest. As the most usual NMR technique, the spin echo formation will be examined in the next subsection.

\section{B. Application to spin echoes}

In the classical Hahn's experiment (Hahn, 1950), the $180^{\circ} \mathrm{RF}$ pulse is emitted after time $T / 2$ to invert the spin magnetizations (Fig. 1(a)). For immobile spins, dephasing during the time interval $[0, T / 2]$ is completely compensated by rephasing during the following time interval $[T / 2, T]$, if the applied magnetic field satisfies the rephasing condition

$$
\int_{0}^{T / 2} d t B_{t}(\mathbf{r})-\int_{T / 2}^{T} d t B_{t}(\mathbf{r})=0
$$

at any spin location $\mathbf{r}$ inside $\Omega$. In this case, the accumulated phase $\varphi$ is strictly zero for all spins (i.e., their magnetizations are in phase at time $T$ ), which leads to echo formation at time $T$. When spins diffuse, rephasing is not complete, and the echo amplitude is decreased. This attenuation can be experimentally measured to study the spin motion.

In a typical situation, the magnetic field $B_{t}(\mathbf{r})$ is composed of a constant field $B_{0}$ generated by a static magnet, and a time-depending diffusion-sensitizing inhomogeneous field $\beta F(t) B(\mathbf{r})$ of maximum intensity $\beta$. The temporal and spatial profiles, $F(t)$ and $B(\mathbf{r})$, are defined to be dimensionless and normalized to 1 . For mathematical convenience, the temporal profile is supposed to be a piecewise-smooth function on the interval $[0, T]$, while the spatial profile is a smooth function in the bulk (domain $\Omega$ ). These formal assumptions are fulfilled in practice.

Throughout this paper, the application of the $180^{\circ} \mathrm{RF}$ pulse will be taken into account by inverting the sign of the function $F(t)$ for $t>T / 2$. The above rephasing condition is reformulated for such an "effective" temporal profile of the magnetic field as

$$
\int_{0}^{T} d t F(t)=0 .
$$

Since the contribution of the constant field $B_{0}$ vanishes after rephasing, the total phase $\varphi$, accumulated during the time $T$, can be written in the same form as before, with $B_{t}(\mathbf{r})=\beta F(t) B(\mathbf{r})$ :

$$
\varphi=\gamma \int_{0}^{T} d t \beta F(t) B(\mathbf{r}(t)) .
$$

In Eqs. (30), (32), the time dependence of the matrix $\mathcal{B}(t)$ and vector $\hat{U}(t)$ can thus be factorized out:

$$
\mathcal{B}(t)=F(t) \mathcal{B} \quad \hat{U}(t)=F(t) \hat{U},
$$

where

$$
\begin{aligned}
\mathcal{B}_{m, m^{\prime}} & =\int_{\Omega} d \mathbf{r} u_{m}^{*}(\mathbf{r}) B(\mathbf{r}) u_{m^{\prime}}(\mathbf{r}), \\
\hat{U}_{m} & =V^{-1 / 2} \int_{\Omega} d \mathbf{r} u_{m}^{*}(\mathbf{r}) B(\mathbf{r}) .
\end{aligned}
$$

For convenience, the integral variable $t$ can be replaced by a dimensionless parameter $t / T$ :

$$
\varphi=\gamma \beta T \int_{0}^{1} d t f(t) B\left(X_{t}\right),
$$

where

$$
X_{t} \equiv \mathbf{r}(t T) \quad \text { and } \quad f(t) \equiv F(t T)
$$

correspond to the time rescaling of the reflected Brownian motion and the effective temporal profile. In the remainder of this paper, $t$ will denote the dimensionless 
time ranging between 0 and 1 . The dimensionless parameter

$$
q=\gamma \beta T
$$

gives the effective dephasing of spins with gyromagnetic ratio $\gamma$ in a magnetic field of strength $\beta$ applied over time $T$. The two independent parameters $p$ and $q$ will result different NMR regimes for restricted diffusion.

According to (41), the physical phase $\varphi$ is proportional to the random variable

$$
\phi=\int_{0}^{1} d t f(t) B\left(X_{t}\right)
$$

which we shall still call "phase". Relation (2) shows that the macroscopic signal $E$ is in fact the characteristic function of this variable:

$$
E=\mathbb{E}\left\{e^{i q \phi}\right\}
$$

Since phase $\phi$ is a bounded random variable, its characteristic function is known to be analytical (Feller, 1971). In particular, its series expansion

$$
E=\sum_{n=0}^{\infty} \frac{(i q)^{n}}{n !} \mathbb{E}\left\{\phi^{n}\right\}
$$

absolutely converges for any $q$. The moments $\mathbb{E}\left\{\phi^{n}\right\}$ may be straightforwardly deduced from the results of Sec. III.A:

$$
\mathbb{E}\left\{\frac{\phi^{n}}{n !}\right\}=<\mathbb{E}\left\{B\left(X_{t_{1}}\right) \ldots B\left(X_{t_{n}}\right)\right\}>_{n},
$$

where $\langle\ldots\rangle_{n}$ denotes the $f$-weighted (ordered) time average of the magnetic field, with the effective temporal profile $f(t)$. For any function $h\left(t_{1}, \ldots, t_{n}\right)$ of time variables $t_{1}, \ldots t_{n}$, this average is defined as

$$
<h\left(t_{1}, \ldots, t_{n}\right)>_{n}=\int_{0}^{1} d t_{1} \ldots \int_{t_{n-1}}^{1} d t_{n} f\left(t_{1}\right) \ldots f\left(t_{n}\right) h\left(t_{1}, \ldots, t_{n}\right) .
$$

For example, one has

$$
<\left(t_{1}-t_{2}\right)>_{2}=\int_{0}^{1} d t_{1} f\left(t_{1}\right) \int_{t_{1}}^{1} d t_{2} f\left(t_{2}\right)\left(t_{1}-t_{2}\right) .
$$

The multiple correlation function $\mathbb{E}\left\{B\left(X_{t_{1}}\right) \ldots B\left(X_{t_{n}}\right)\right\}$ represents the spatial average of the reflected Brownian motion, "weighted" by the magnetic field profile $B(\mathbf{r})$. It can be written according to (33) in the matrix form:

$$
\begin{aligned}
& \mathbb{E}\left\{B\left(X_{t_{1}}\right) \ldots B\left(X_{t_{n}}\right)\right\}= \\
& U \cdot\left(\left[\prod_{j=1}^{n-1} e^{-p \Lambda t_{j}} \mathcal{B} e^{p \Lambda t_{j}}\right] e^{-p \Lambda t_{n}} \hat{U}\right) .
\end{aligned}
$$

The multiple correlation function does not depend on the temporal profile $f(t)$ of the magnetic field. The crucial advantage of the last relation in comparison with Eq. (33) is that the temporal and spatial averages can now be calculated separately. First, the matrix $\mathcal{B}$ is constructed for a given domain $\Omega$ and spatial profile $B(\mathbf{r})$, independently of the function $f(t)$. Second, the $f$-weighted time average of the multiple correlation function is calculated. This is a significant simplification for the theoretical analysis and numerical computation.

In the case of Neumann boundary condition (11), there is no loss of magnetization at the interface, so that its steady state distribution does exist and has to be uniform. It means that

- for any geometry of the domain $\Omega$, the ground eigenmode corresponds to the constant eigenfunction $u_{0}(\mathbf{r})=V^{-1 / 2}$, with eigenvalue $\lambda_{0}=0$;

- the initial density of spins is typically uniform, ${ }^{8}$ $\rho(\mathbf{r})=1 / V$.

In this case, the definition (31) of the vector $U$ implies $U_{m}=\delta_{m, 0}$. Moreover, the first diagonal element of the matrix $e^{-p \Lambda t_{1}}$ in Eq. (50) is equal to 1, independently of $p$ and $t_{1}$. Finally, the elements $\hat{U}_{m}$ of the vector $\hat{U}$ can be written as $\mathcal{B}_{m, 0}$. All these simplifications lead to the following expression for the multiple correlation functions in the case of reflecting boundaries:

$$
\begin{aligned}
& \mathbb{E}\left\{B\left(X_{t_{1}}\right) \ldots B\left(X_{t_{n}}\right)\right\}= \\
& {\left[\mathcal{B} e^{-p\left(t_{2}-t_{1}\right) \Lambda} \mathcal{B} e^{-p\left(t_{3}-t_{2}\right) \Lambda} \mathcal{B} \ldots \mathcal{B} e^{-p\left(t_{n}-t_{n-1}\right) \Lambda} \mathcal{B}\right]_{0,0}}
\end{aligned}
$$

where the subscript 0,0 denotes the first diagonal element of the matrix product in brackets. In the next sections, we shall show how this representation can be applied to derive different NMR regimes of restricted diffusion. For sake of clarity, we focus our attention on the reflecting boundary condition, bearing in mind that taking into account surface relaxation effects is in general straightforward but more cumbersome.

\footnotetext{
8 At equilibrium condition, the initial spin density $\rho(\mathbf{r})$ is uniform for reflecting boundaries. Usually, it is considered that the $90^{\circ}$ $\mathrm{RF}$ pulse acts uniformly over the sample so that all spins are excited in the same way. Consequently, the initial magnetization $\mathfrak{m}(\mathbf{r}, t=0)$ is proportional to $\rho(\mathbf{r})$ and thus uniform. However, this is not always the case. Recently, Song (2000); Song et al. (2000, 2003) proposed an efficient experimental technique to detect the pore geometry, when the uniformly distributed spins are excited selectively within a narrow frequency range that creates a non-uniform magnetization at time $t=0$. In MCF approach, this effect can be taken into account through the vector $U$.
} 


\section{Temporal profiles of the magnetic field}

The application of two identical linear gradients of duration $\delta \leq 1 / 2$ before and after the $180^{\circ} \mathrm{RF}$ pulse is probably the most common way to encode the diffusive motion. The temporal profile of these pulses (i.e., their "shape") is typically trapezoidal in an experiment and rectangular for theoretical analysis (Fig. 2(c),(d)) like

$$
f(t)=\Theta(t)-\Theta(t-\delta)-\Theta(t-1 / 2)+\Theta(t-\delta-1 / 2),
$$

where $\Theta(t)$ is the Heaviside step function, $\Theta(t)=1$ for $t>0$, and 0 otherwise. Among others, two specific cases were particularly favored by theoreticians. These are the steady profile $(\delta=1 / 2)$ employed in the first spin-echo experiment by Hahn (1950) and the narrow-pulse profile $(\delta \ll 1 / 2)$ first introduced by Tanner and Stejskal (1968). Whatever the value of $\delta$ is, the function (52) will be called "Stejskal-Tanner temporal profile". A train of $2 k$ pulses of alternate signs can be treated in a similar way (Fig. 2(b)). This would correspond to a Carr-PurcellMeiboom-Gill sequence which is also commonly used to experimentally study restricted diffusion.

Another interesting situation occurs when the magnetic field is applied periodically with time. In this case, one samples molecular dynamics in the frequency domain rather than in the time domain. Callaghan and Stepišnik $(1995,1996)$ showed that such frequency modulated gradient spin-echo measurements provide access to much shorter time scales than that of the traditional Stejskal-Tanner profile. We shall consider sinusoidal and cosinusoidal temporal profiles, $f(t)=\sin (2 \pi k t)$ and $f(t)=\cos (2 \pi k t)$, where $k$ is the number of periods until echo formation at time $t=1$ (Fig. 2(e)).

\section{Spatial profiles of the magnetic field}

\section{Linear gradient}

The simplest case of a linear magnetic field gradient covers the majority of theoretical, numerical and experimental NMR studies of the diffusive motion. If nonlinear effects can be neglected, the magnetic field of a linear gradient $g$ is proportional to the projection of the coordinate vector $\mathbf{r}$ onto the gradient direction $\mathbf{e}_{g}$, i.e., $\beta=g L$ and

$$
B(\mathbf{r})=\left(\mathbf{e}_{g} \cdot \mathbf{r}\right) / L .
$$

The dimensionless magnetic field strength $q$ is then:

$$
q=\gamma g T L
$$

The substitution of the linear magnetic field gradient (53) into (39) leads to a specific form for the matrix $\mathcal{B}$ which was first introduced by Robertson (1966) and then extended by Barzykin $(1998,1999)$ to describe the signal attenuation due to diffusive motion. (a)

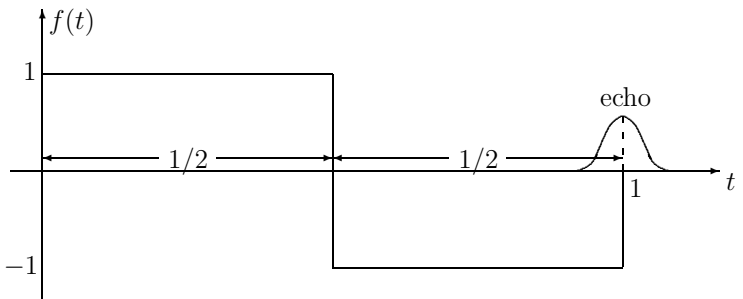

(b)

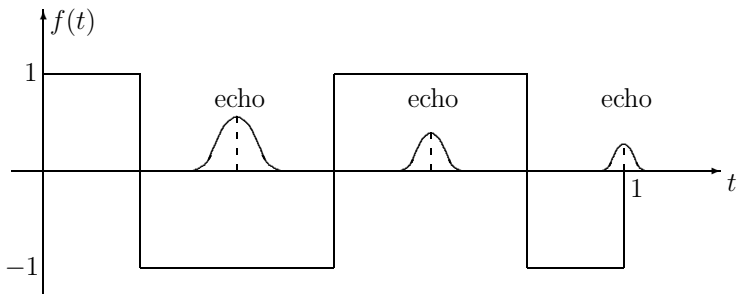

(c)

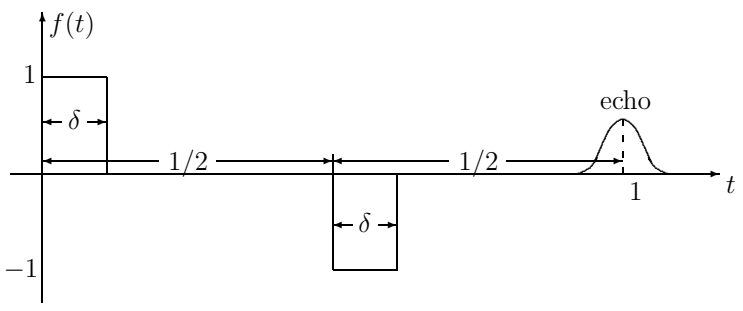

(d)

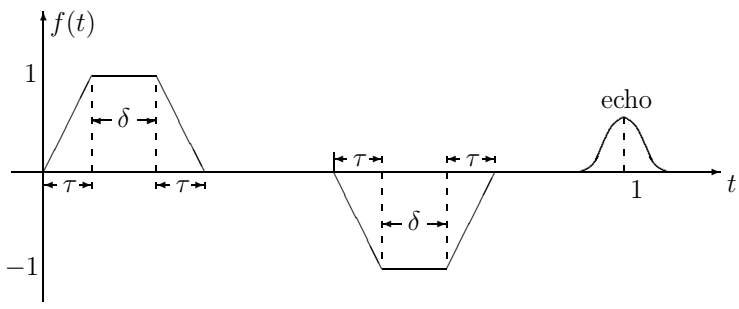

(e)

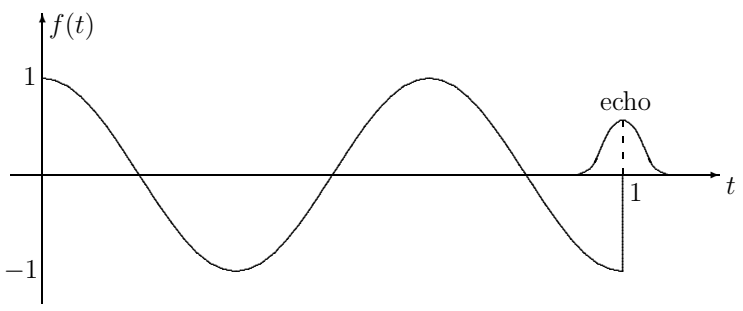

FIG. 2 Several effective temporal profiles $f(t)$ : steady (a), CPMG (b), Stejskal-Tanner rectangular (c) and trapezoidal (d), and periodic (e). Profiles (a)-(c) correspond to the gradients shapes shown in Fig. 1 (for convenience, the rectangular profile (c) was shifted to the left). The case (e) is related to a frequency modulated magnetic field. The inversion of the spin magnetization by the $180^{\circ} \mathrm{RF}$ pulse is already taken into account by changing the sign of the effective profile.

\section{Parabolic magnetic field}

For a long time, nonlinear magnetic fields were practically excluded from the usual scope of scientific questions both for theoreticians and experimentalists. These fields may have different origins like hardware imperfections or susceptibility effects. In the former case, an applied linear gradient is known to be uniform only near the center 
of the coils (Tarczon and Halperin, 1985). If the sample is relatively big, its peripheric regions are subjected to a nonlinear magnetic field. In turn, a difference between the magnetic susceptibility of the interface (e.g., pore materials, rocks, alveolar tissues) and that of the bulk (e.g., water or oil molecules, gases) leads to local non-linear magnetic field inhomogeneities (Bergman and Dunn, 1995; Callaghan, 1991; Kleinberg and Horsfield, 1990; Majumdar and Gore, 1988). These fields were difficult to control experimentally and to investigate theoretically by classical approaches.

The growing interest to nonlinear magnetic fields was brought by Le Doussal and Sen (1992) thanks to an exact resolution of the Bloch-Torrey equation in the whole space in the presence of a steady parabolic magnetic field. Note that such a field can be experimentally generated (Bendel, 1990). Other important examples of nonlinear fields can be found in stray field experiments (Hürlimann, 2001) or geophysical applications (Kleinberg et al., 1992).

In this review, the normalized isotropic parabolic magnetic field

$$
B(\mathbf{r})=\mathbf{r}^{2} / L^{2}
$$

is considered as a paradigm for nonlinear fields. Its intensity $g_{2}$ determines the dimensionless magnetic field strength $q$ :

$$
q=\gamma g_{2} T L^{2}
$$

The implementation of the other possible choices of magnetic field spatial profile is straightforward, at least at the numerical level. In particular, Zielinski and Sen (2000) paid special attention to a cosine profile of the magnetic field in the one-dimensional case because it can be thought as a crude model for microscopic field inhomogeneities induced by susceptibility differences. This interesting choice of the spatial profile, as well as a number of others, is not considered in this paper, but can be treated within the present approach.

\section{E. Basic confining domains}

Before proceeding with the analysis of the moments $\mathbb{E}\left\{\phi^{n}\right\}$, we give three examples of confining media for which the eigenbasis of the Laplace operator is explicitly known (Carslaw and Jaeger, 1959; Crank, 1975). The majority of theoretical studies are actually restricted to these "basic" domains. Although their shapes are quite simple, the use of these structures to model a geometrical confinement considerably helped to comprehend the diffusive motion in more realistic media.

\section{Slab}

The restricted diffusion between two parallel infinite planes separated by a unit distance is the most studied case. This problem is equivalent to one-dimensional diffusion on the interval $(0,1)$ with reflections at endpoints 0 and 1 . The eigenbasis of the Laplace operator with Neumann boundary condition (11) is simple and well known:

$$
\lambda_{m}=\pi^{2} m^{2}, \quad u_{m}(x)=\epsilon_{m} \cos (\pi m x),
$$

where $\epsilon_{m}=\sqrt{2}$ for $m>0$ and $\epsilon_{0}=1$ to normalize the eigenfunctions $u_{m}(x)$. The eigenvalues $\lambda_{m}$ directly give the elements of the matrix $\Lambda$.

The elements of the matrix $\mathcal{B}$ depend on the choice of the spatial profile $B(x)$ of the magnetic field. In the case of a linear gradient applied in the direction normal to the planes, $B(x)=x$, one easily calculates

$$
\mathcal{B}_{m, m^{\prime}}=\frac{\epsilon_{m} \epsilon_{m^{\prime}}}{\pi^{2}}\left((-1)^{m+m^{\prime}}-1\right) \frac{m^{2}+m^{\prime 2}}{\left(m^{2}-m^{\prime 2}\right)^{2}}
$$

for $m \neq m^{\prime}$, and $\mathcal{B}_{m, m}=1 / 2$. In particular, one gets for $m>0$

$$
\mathcal{B}_{0, m}=\sqrt{2}\left((-1)^{m}-1\right) \lambda_{m}^{-1} .
$$

For the parabolic magnetic field (55), one finds

$\mathcal{B}_{m, m^{\prime}}= \begin{cases}\frac{2 \epsilon_{m} \epsilon_{m^{\prime}}(-1)^{m+m^{\prime}}\left(m^{2}+m^{\prime 2}\right)}{\pi^{2}\left(m^{2}-m^{\prime 2}\right)^{2}}, & m \neq m^{\prime}, \\ \frac{1}{3}+\frac{1}{2 \pi^{2} m^{2}}, & m=m^{\prime}>0,\end{cases}$

and $\mathcal{B}_{0,0}=1 / 3$. In particular, one has

$$
\mathcal{B}_{0, m}=\frac{2 \sqrt{2}(-1)^{m}}{\pi^{2} m^{2}} .
$$

Another choice of the magnetic field spatial profile can be implemented straightforwardly.

If the surface relaxation is significant, the Neumann boundary condition (11) can be replaced by a more general Fourier boundary condition (10). It is convenient to introduce the dimensionless relaxation rate $h$ as

$$
h=K L / D,
$$

where $L$ is the physical length of the interval. The eigenvalues and eigenfunctions of the Laplace operator have to be recalculated ${ }^{9}$ (see Table I). Although the expressions become more cumbersome and less transparent, the

\footnotetext{
9 The matrix $\mathcal{B}$ was calculated for a linear magnetic field gradient in basic domains by Barzykin (1999). This computation is elementary for a slab geometry since the eigenbasis is formed by sine and cosine functions. Please note that the relations presented here correspond to restricted diffusion in a segment $(0,1)$, while Barzykin considered a centered segment $(-1 / 2,1 / 2)$.
} 


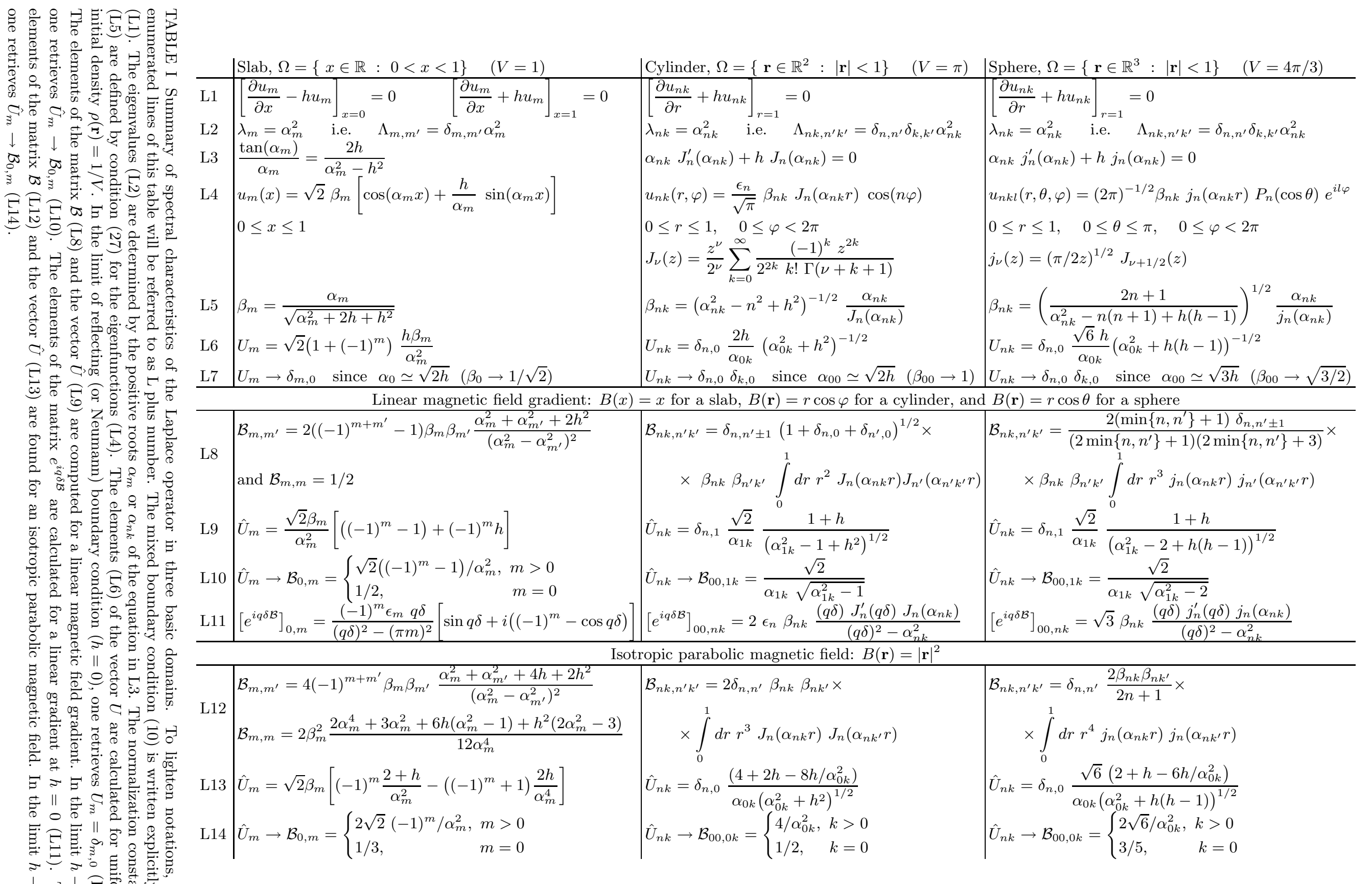


same concept is applied. We should mention, however, two differences with respect to the reflecting case. First, one has to use the general relation (50) for the multiple correlation functions instead of its simpler form (51). In particular, the computation of the vectors $U$ and $\hat{U}$ is required (see Table I). Second, the matrices $\mathcal{B}$ and $\Lambda$ depend on the dimensionless relaxation rate $h$. If this value is fixed, there is no conceptual difference with respect to the case $h=0$. If, on the opposite, one studies the dependence of the signal (or correlation functions) on this parameter, the matrices $\mathcal{B}$ and $\Lambda$ have to be recalculated for each value of $h$. From the numerical point of view, this is not a problem since their numerical computation is quite rapid. However, theoretical analysis of the dependence on $h$ is difficult since this parameter intervenes the eigenvalues and eigenfunctions of the Laplace operator in a complex manner.

\section{Cylinder}

For a cylinder of unit ${ }^{10}$ radius, the classical representation of the eigenfunctions involve two positive indices $n$ and $k$

$$
u_{n k}(r, \varphi)=\frac{\epsilon_{n}}{\sqrt{\pi}} \beta_{n k} J_{n}\left(\alpha_{n k} r\right) \cos (n \varphi),
$$

where $J_{n}(z)$ are the Bessel functions of the first kind. The normalization constants $\beta_{n k}$ and the positive roots $\alpha_{n k}$ are defined in Table I. In our notation, the pair of indices $n$ and $k$ can still be thought as a single index $m$. For a numerical implementation, one has to sort the eigenvalues $\lambda_{n k}=\alpha_{n k}^{2}$ in an ascending order to truncate the infinite-dimension matrices $\mathcal{B}$ and $\Lambda$. The position of the eigenmode in such a sequence can be used for its single index $m$.

The calculation of the matrix $\mathcal{B}$ and vector $U$ for a linear magnetic field gradient was performed by Barzykin (1999). His results and our calculation for a parabolic magnetic field are summarized in Table I. Please note that the dimensionless relaxation rate $h$ is still given by (62), where $L$ is the physical radius of the cylinder.

The finding of the roots $\alpha_{n k}$ and integration over $r$ in the above expressions require a numerical computation

10 The physical radius $L$ will re-appear in dimensionless coefficients $p, q$ and $h$. At this point, we should stress that the term "characteristic dimension of the confining medium" is a bit ambiguous. For example, if one considers restricted diffusion in a long thin tube, the motion is essentially confined along the transverse direction (perpendicular to the axis of the tube). In this case, the "characteristic dimension" means the width, and not the height of the tube. The situation may be still more complicated in porous media when different length scales present. We shall not discuss these subtle points here, staying on a formal position when $L$ can be thought as an appropriate length scale to get dimensionless parameters $q$ and $p$. For a slab geometry, $L$ is always the separation width of parallel plates (length of an interval). For a cylinder and a sphere, $L$ is always the radius. that is simple and has to be performed only once ${ }^{11}$ for a chosen spatial profile of the magnetic field. The stored matrices $\mathcal{B}$ and $\Lambda$ can then be used for subsequent investigations of restricted diffusion.

\section{Sphere}

For a sphere of unit radius, the eigenfunctions are

$$
u_{n k l}(r, \theta, \varphi)=(2 \pi)^{-1 / 2} \beta_{n k} j_{n}\left(\alpha_{n k} r\right) P_{n}(\cos \theta) e^{i l \varphi},
$$

where $P_{n}(x)$ the Legendre polynomials. The normalization constants $\beta_{n k}$ and the positive roots $\alpha_{n k}$ are defined in Table I. Although the eigenfunctions are formally enumerated by triple index $\{n, k, l\}$, the last index $l$ will be omitted throughout this paper since the polar coordinate $\varphi$ is not involved in the following analysis. In particular, the integration over $\varphi$ will simply give a factor $2 \pi$. If the spatial profile of the magnetic field was dependent on $\varphi$, this coordinate would be taken into account.

The calculation of the matrix $\mathcal{B}$ and vector $U$ for a linear magnetic field gradient was performed by Barzykin (1999). His results and our calculation for a parabolic magnetic field are summarized in Table I. Please note that the dimensionless relaxation rate $h$ is still given by (62), where $L$ is the physical radius of the sphere.

As previously, the elements of the matrices $\mathcal{B}$ and $\Lambda$ have to be numerically computed only once, and then their stored values can be used for further analysis of restricted diffusion in a sphere. In what follows, we mainly focus on the reflecting boundary condition, while the accounting for a surface relaxation is straightforward but more cumbersome.

\section{SLOW DIFFUSION REGIME $(p \ll 1)$}

The multiple correlation function approach is developed as a mathematical basis for a theoretical study of restricted diffusion. To show its efficiency, we are going to retrieve some classical results in a more general form. We start by the analysis of the moments $\mathbb{E}\left\{\phi^{n}\right\}$ in the slow diffusion regime, when the dimensionless diffusion coefficient $p$ goes down to 0 . This regime is also known as the "short time limit" since $T \rightarrow 0$ implies $p \rightarrow 0$. First, we calculate the leading term of the second moment and suggest its general form for higher order moments. Then corrections to the leading term are discussed.

11 The situation is more difficult when one studies the dependence on the surface relaxation $h$. In this case, one has to recalculate the matrices for each value of $h$. 


\section{A. Leading term of the second moment}

According to the general relation (51), the second moment of the random phase $\phi$ can be written as

$$
\mathbb{E}\left\{\frac{\phi^{2}}{2}\right\}=<\left[\mathcal{B} e^{-p\left(t_{2}-t_{1}\right) \Lambda} \mathcal{B}\right]_{0,0}>_{2} .
$$

In the limit $p \rightarrow 0$, one can formally expand the exponential function in a power series up to the first order:

$$
\mathbb{E}\left\{\frac{\phi^{2}}{2}\right\}=<1>_{2} \tilde{\zeta}_{0}-p<\left(t_{2}-t_{1}\right)>_{2} \zeta_{1}+\ldots,
$$

where $f$-weighted time averages $\langle 1\rangle_{2}$ and $\left\langle\left(t_{2}-t_{1}\right)\right\rangle_{2}$ are defined by Eq. (48), while the coefficients $\zeta_{k}$ denote the following spatial averages:

$$
\zeta_{k}=\sum_{m=1}^{\infty} \mathcal{B}_{0, m} \lambda_{m}^{k} \mathcal{B}_{m, 0}
$$

and

$$
\tilde{\zeta}_{0}=\zeta_{0}+\left(\mathcal{B}_{0,0}\right)^{2}
$$

Note that the above expansion is formal since the higher order terms $\left(\mathcal{B} \Lambda^{2} \mathcal{B}, \mathcal{B} \Lambda^{3} \mathcal{B}, \ldots\right)$ diverge strictly speaking (this divergence can be renormalized as discussed in the next subsections).

The coefficients $\tilde{\zeta}_{0}$ and $\zeta_{1}$ can be further simplified by considering the spatial averages $\left[\mathcal{B}^{2}\right]_{0,0}$ and $[\mathcal{B} \Lambda \mathcal{B}]_{0,0}$ in view of a field theory technique. Indeed, the matrix $\mathcal{B}$ contains and represents a scalar field $B(\mathbf{r})$, while the matrix $\Lambda$ acts like a field operator: one can replace the combination $\lambda_{m} u_{m}(\mathbf{r})$ by $-L^{2} \Delta u_{m}(\mathbf{r})$, and then apply the second Green formula to transpose the Laplace operator to the adjacent field $B(\mathbf{r})$. For example, one starts from the definition

$$
\tilde{\zeta}_{0}=\frac{1}{V} \sum_{m=0}^{\infty} \int_{\Omega} d \mathbf{r} u_{m}(\mathbf{r}) B(\mathbf{r}) \int_{\Omega} d \mathbf{r}^{\prime} u_{m}^{*}\left(\mathbf{r}^{\prime}\right) B\left(\mathbf{r}^{\prime}\right)
$$

and obtains

$$
\tilde{\zeta}_{0}=\frac{1}{V} \int_{\Omega} d \mathbf{r} B^{2}(\mathbf{r})
$$

since there was no matrix $\Lambda$ to act on the field $B(\mathbf{r})$, while the summation over $m$ gave $\delta\left(\mathbf{r}-\mathbf{r}^{\prime}\right)$. Note that the coefficient $\tilde{\zeta}_{0}$ does not contribute to the second moment since the $f$-weighted time average of a constant vanishes due to the rephasing condition (36).

In a similar way, one writes the definition of the coefficient $\zeta_{1}$ and obtains by the second Green formula

$$
\begin{gathered}
\zeta_{1}=\frac{L^{2}}{V} \sum_{m=1}^{\infty}\left(\int_{\Omega} d \mathbf{r} u_{m}(\mathbf{r}) \Delta B(\mathbf{r})-\int_{\partial \Omega} d \mathbf{r} u_{m}(\mathbf{r}) \frac{\partial B}{\partial n}\right) \\
\times \int_{\Omega} d \mathbf{r}^{\prime} u_{m}^{*}\left(\mathbf{r}^{\prime}\right) B\left(\mathbf{r}^{\prime}\right),
\end{gathered}
$$

where the boundary condition $\partial u_{m}(\mathbf{r}) / \partial n=0$ cancelled another integral over the boundary $\partial \Omega$. The summation over $m$ gives $\delta\left(\mathbf{r}-\mathbf{r}^{\prime}\right)-1 / V$, whence one gets

$$
\zeta_{1}=\frac{L^{2}}{V} \int_{\Omega} d \mathbf{r}|\nabla B(\mathbf{r})|^{2},
$$

where $\nabla B(\mathbf{r})$ is the gradient of the magnetic field $B(\mathbf{r})$. In particular, for the linear gradient profile (53), the spatial average $\zeta_{1}$ is equal to 1 for any confining geometry.

The leading term of the second moment is then

$$
\mathbb{E}\left\{\frac{\phi^{2}}{2}\right\} \simeq p<\left(t_{1}-t_{2}\right)>_{2}\left(\frac{L^{2}}{V} \int_{\Omega} d \mathbf{r}|\nabla B(\mathbf{r})|^{2}\right) .
$$

This is an extension of the slow diffusion results to arbitrary temporal and spatial profiles of the magnetic field. In the particular case of Stejskal-Tanner temporal profile (52), one has

$$
<\left(t_{1}-t_{2}\right)>_{2}=\delta^{2}(1 / 2-\delta / 3),
$$

and Eq. (73) is reduced to the result of Tarczon and Halperin (1985) derived for the restricted diffusion between two parallel planes by Fourier expansion of the magnetic field.

\section{B. Higher order moments}

A similar analysis can be in principle realized to calculate the leading terms for higher moments of even order. However, the computation becomes much more cumbersome, since a large number of particular cases have to be carefully considered. Moreover, an unbounded increase of the elements $\Lambda_{m, m}$ of the matrix $\Lambda$ when $m \rightarrow \infty$ requires a renormalization procedure. The calculation of the leading term of the fourth moment is given in Appendix A:

$$
\mathbb{E}\left\{\frac{\phi^{4}}{4 !}\right\} \simeq \frac{p^{2}}{2}\left(<\left(t_{1}-t_{2}\right)>_{2}\right)^{2}\left(\frac{L^{4}}{V} \int_{\Omega} d \mathbf{r}|\nabla B(\mathbf{r})|^{4}\right) .
$$

Looking at this relation, one can suggest a general form of the leading terms for even order moments in the slow diffusion regime:

$$
\mathbb{E}\left\{\frac{\phi^{2 n}}{(2 n) !}\right\} \simeq \frac{p^{n}}{n !}\left(<\left(t_{1}-t_{2}\right)>_{2}\right)^{n} \frac{L^{2 n}}{V} \int_{\Omega} d \mathbf{r}|\nabla B(\mathbf{r})|^{2 n} .
$$

A systematic computational technique will be of great interest to rigorously demonstrate this relation.

In the particular case of a linear magnetic field gradient, one has $L^{2 n}|\nabla B(\mathbf{r})|^{2 n}=1$, and

$$
\mathbb{E}\left\{\frac{\phi^{2 n}}{(2 n) !}\right\} \simeq \frac{1}{n !}\left(\mathbb{E}\left\{\frac{\phi^{2}}{2}\right\}\right)^{n}
$$


where the leading term of the second moment is simply

$$
\mathbb{E}\left\{\frac{\phi^{2}}{2}\right\} \simeq p<\left(t_{1}-t_{2}\right)>_{2} .
$$

Substituting the leading terms (77) in the series expansion (46), one finds a compact form of the Stejskal-Tanner formula (7)

$$
E \simeq \exp \left[-q^{2} p<\left(t_{1}-t_{2}\right)>_{2}\right]
$$

because the $f$-weighted time average $\left\langle\left(t_{1}-t_{2}\right)\right\rangle_{2}$ can be written in a more usual way as:

$$
<\left(t_{1}-t_{2}\right)>_{2}=\int_{0}^{1} d t\left(\int_{0}^{t} d t^{\prime} f\left(t^{\prime}\right)\right)^{2} .
$$

For example, it is equal to $1 / 12$ for the steady temporal profile, leading to a widely used expression (3).

Interestingly, the relation (77) becomes exact in the free (or unrestricted) diffusion limit, when the characteristic dimension $L$ of the confining domain goes to infinity. In this case, the dimensionless diffusion coefficient $p$ tends to 0 , while the dimensionless magnetic field strength $q$ diverges but their combination $q^{2} p$ remains constant. Since the moment $\mathbb{E}\left\{\phi^{2 n}\right\}$ appears in front of $q^{2 n}$, its leading term of order $p^{n}$ gives a nontrivial contribution, while the correction terms of order higher than $p^{n}$ vanish. As a consequence, the Gaussian form (79) is exact for a linear magnetic field gradient in the free diffusion limit. It is worth noting that the passage from restricted to unrestricted diffusion is in general more delicate than here (see subsection VI.G).

For nonlinear magnetic fields, the substitution of the moments (76) in the series expansion (46) gives

$$
E \simeq \frac{1}{V} \int_{\Omega} d \mathbf{r} \exp \left[-q^{2} p<\left(t_{1}-t_{2}\right)>_{2} L^{2}|\nabla B(\mathbf{r})|^{2}\right] .
$$

This relation can be seen as an extension of the local gradient approximation (19) by Tarczon and Halperin (1985) to arbitrary temporal profile $f(t)$. Moreover, the present derivation is not restricted to the one-dimensional case. However, this extension is based on the conjectural expression (76) which was proven in Appendix A only for $n=2$. The MCF approach appears as an appropriate basis to demonstrate this result for any order $n$. At the same time, the relation (81) remains an approximation involving the leading terms of all even moments in the slow diffusion regime. Note that the free diffusion limit $(L \rightarrow \infty)$ of Eq. (81) in the case of nonlinear magnetic fields may not exist (or be trivial).

We should stress that there is a significant difference between the slow and free diffusion regimes, although in both cases $p$ goes to 0 . In the free diffusion limit $(L \rightarrow \infty$ and $q \rightarrow \infty$ ), each combination $\left(q^{2} p\right)^{n}$ provides a nontrivial contribution, while the correction terms for each moment vanish. Brought together, these contributions lead to the Gaussian form (79). In the slow diffusion limit ( $L$ and $q$ are kept fixed), the second moment of order $p$ gives a major contribution, while the other moments of higher orders appear as its vanishing corrections, and can thus be neglected. Consequently, the series expansion (46) of the signal becomes

$$
E \simeq 1-q^{2} \mathbb{E}\left\{\phi^{2} / 2\right\}
$$

or, with the same accuracy,

$$
E \simeq \exp \left[-q^{2} \mathbb{E}\left\{\phi^{2} / 2\right\}\right]
$$

Although one retrieved again the Gaussian form (79), this relation is just a convenient representation of the first order approximation (82). In the literature, there are a lot of speculations about the slow diffusion approximation (83) which is actually trivial. The same relation can be written in the limit $q \rightarrow 0$ with a fixed $p$. In both cases, its applicability is limited to relatively small values of $q$ and $p$. A potential extension of its validity is called the Gaussian phase approximation. In contrast, the very same relation (79) is exact in the free diffusion limit for any values of $p$ and $q$. The confusion between the free and slow diffusion regimes is quite common and may be misleading. The presence of a non-uniform magnetic field yields that extending the confining domain is not equivalent to reducing the diffusion length.

\section{Correction term to the second moment}

A careful revision of the derivation in subsection IV.A would reveal a gross defect. As we mentioned at the beginning of this section, the series expansion of the exponential function in (65) is not mathematically allowed since the terms $\mathcal{B} \Lambda^{2} \mathcal{B}, \mathcal{B} \Lambda^{3} \mathcal{B}, \ldots$ are divergent due to an unbounded increase of the elements $\Lambda_{m, m}$ with $m$. At the same time, this very same increase ensures a rapid convergence of the exponential function $\exp \left[-p\left(t_{2}-t_{1}\right) \Lambda\right]$ itself. A certain renormalization procedure has thus to be introduced.

Let us consider again the second moment

$\left.\mathbb{E}\left\{\frac{\phi^{2}}{2}\right\}=\sum_{m=0}^{\infty} \mathcal{B}_{0, m} \mathcal{B}_{m, 0} \sum_{n=0}^{\infty} \frac{(-p)^{n}}{n !} \lambda_{m}^{n}<\left(t_{2}-t_{1}\right)^{n}\right\rangle_{2}$.

To extract the coefficient in front of $p^{n}$, one has to exchange the order of summation. However, this operation is not allowed since it would lead to a divergent series. As one will see below, this difficulty can be formally overcome by taking the sum over $m$ up to a large but finite cut-off $M$ :

$$
\mathbb{E}\left\{\frac{\phi^{2}}{2}\right\} \simeq \sum_{n=0}^{\infty} \frac{(-p)^{n}}{n !}<\left(t_{2}-t_{1}\right)^{n}>_{2}\left[\sum_{m=0}^{M} \mathcal{B}_{0, m} \mathcal{B}_{m, 0} \lambda_{m}^{n}\right] .
$$

As we have already seen, the zeroth term $(n=0)$ vanishes after the $f$-weighted time average, while the first 
term $(n=1)$ converges as $M$ goes to infinity and provides the leading contribution of order $p$. In contrast, the higher order terms $(n \geq 2)$ are divergent in the limit $M \rightarrow \infty$. For three basic domains and two choices of the magnetic field spatial profile, the asymptotic behavior of the divergent sum at large $M$ is

$$
\sum_{m=0}^{M} \mathcal{B}_{0, m} \mathcal{B}_{m, 0} \lambda_{m}^{n} \propto c_{\mathcal{B}} \pi^{2 n-4} \frac{M^{2 n-3}}{2 n-3}
$$

where $c_{\mathcal{B}}$ is a geometry-dependent constant (see Table II). The correction term is then

$$
\frac{1}{2} c_{\mathcal{B}} M^{-3} \sum_{n=2}^{\infty} \frac{\left(-p\left(t_{2}-t_{1}\right) \pi^{2} M^{2}\right)^{n}}{n !(n-3 / 2)}
$$

This series can be calculated explicitly with the help of the identity:

$$
\sum_{n=2}^{\infty} \frac{(-x)^{n}}{n !} \frac{1}{n-3 / 2}=x^{3 / 2} \int_{0}^{x} d \alpha\left(e^{-\alpha}-1+\alpha\right) \alpha^{-5 / 2}
$$

The substitution of $x=p\left(t_{2}-t_{1}\right) \pi^{2} M^{2}$ leads to the correction

$$
\frac{1}{2} c_{\mathcal{B}} p^{3 / 2}\left(t_{2}-t_{1}\right)^{3 / 2} \pi^{3} \Gamma(-3 / 2),
$$

where the integral over $\alpha$ was replaced by its limit $\Gamma(-3 / 2)=4 \sqrt{\pi} / 3$ for $M$ (or $x$ ) going to infinity. The complete expression for the second moment is then

$$
\begin{aligned}
\mathbb{E}\left\{\frac{\phi^{2}}{2}\right\} & \simeq p<\left(t_{1}-t_{2}\right)>_{2}\left(\frac{L^{2}}{V} \int_{\Omega} d \mathbf{r}|\nabla B(\mathbf{r})|^{2}\right) \\
& +p^{3 / 2} \mu_{2}<\left(t_{2}-t_{1}\right)^{3 / 2}>_{2},
\end{aligned}
$$

where

$$
\mu_{2}=\frac{2 c_{\mathcal{B}}}{3 \sqrt{\pi}}
$$

(see Table II). In Appendix B, the Laplace transform technique is used to obtain the $p^{3 / 2}$ dependence and higher order correction terms for restricted diffusion in three basic domains under linear gradient and parabolic magnetic fields. ${ }^{12}$

Although the correction of order $p^{3 / 2}$ vanishes in the limit $p \rightarrow 0$ faster than $p$, it is significant for practical applications (see Fig. 3). This correction is related to

12 We should note, however, that the presence of the $p^{3 / 2}$ correction depends on the spatial profile of the magnetic field. To illustrate this point, let us consider restricted diffusion in a slab with reflecting boundaries under cosinusoidal magnetic field, $B(x)=\cos (\pi k x)$ (integer number $k>0$ is fixed). In this case,

\begin{tabular}{|l|c|c|c|c|}
\hline & & slab & cylinder & sphere \\
\hline \multirow{2}{*}{ linear } & $c_{\mathcal{B}}$ & 4 & 2 & 2 \\
& $\mu_{2}$ & $8 /(3 \sqrt{\pi})$ & $4 /(3 \sqrt{\pi})$ & $4 /(3 \sqrt{\pi})$ \\
\hline \multirow{2}{*}{ parabolic } & $c_{\mathcal{B}}$ & 8 & 16 & 24 \\
& $\mu_{2}$ & $16 /(3 \sqrt{\pi})$ & $32 /(3 \sqrt{\pi})$ & $48 /(3 \sqrt{\pi})$ \\
\hline
\end{tabular}

TABLE II Prefactor $c_{\mathcal{B}}$ and coefficient $\mu_{2}$ in the slow diffusion regime for three basic domains under linear magnetic field gradient and parabolic magnetic field. The values of the coefficient $\mu_{2}$ have been also confirmed by a numerical analysis of the exact relation (65) for these domains.

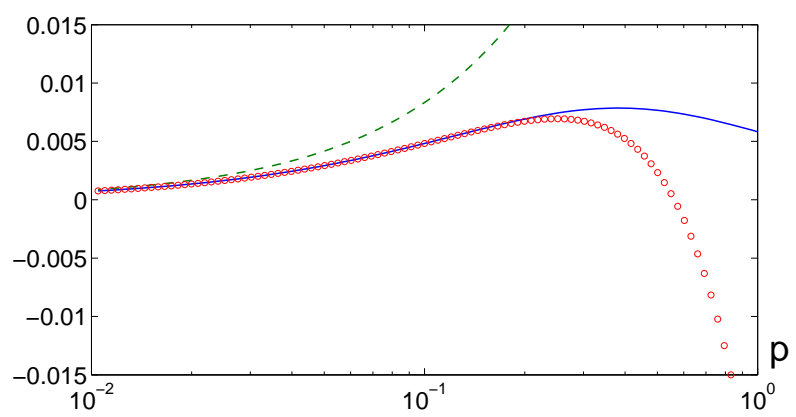

FIG. 3 The second moment $\mathbb{E}\left\{\phi^{2} / 2\right\}$ (solid line) as a function of $p$ for the restricted diffusion between two parallel planes (in a slab) under steady linear gradient magnetic field. For small $p$, this moment is compared to its leading term in the slow diffusion regime with (circles) and without (dashed line) correction term in (90). One clearly sees the importance of this correction.

the fact that the spins in the neighbourhood of the interface, within the diffusion length $(D T)^{1 / 2}$, are more confined than those of the bulk, so that their dephasing is less pronounced. The fraction of these spins can be estimated as $(D T)^{1 / 2} / L$ that explains the additional factor $p^{1 / 2}$ in the $p^{3 / 2}$ dependence. The numerical prefactor $\mu_{2}$ accounts for specific geometry of the confining medium and the spatial profile of the magnetic field, while the $f$ weighted time average $\left\langle\left(t_{2}-t_{1}\right)^{3 / 2}\right\rangle_{2}$ assesses the choice of the temporal profile $f(t)$. In this light, our expression (90) is an extension of the results by Mitra et al. (1992) and by de Swiet and Sen (1994) to the general case of

the matrix $\mathcal{B}$ is particularly simple:

$\mathcal{B}_{m, m^{\prime}}=\frac{\epsilon_{m} \epsilon_{m^{\prime}}}{4}\left(\delta_{k, m+m^{\prime}}+\delta_{k, m-m^{\prime}}+\delta_{k,-m+m^{\prime}}+\delta_{k,-m-m^{\prime}}\right)$.

One then finds the second moment

$$
\mathbb{E}\left\{\phi^{2} / 2\right\}=<\left[\mathcal{B} e^{-p\left(t_{2}-t_{1}\right) \Lambda} \mathcal{B}\right]_{0,0}>_{2}=\frac{1}{2}<e^{-p\left(t_{2}-t_{1}\right) \lambda_{k}}>_{2} .
$$

In the slow diffusion regime $(p \ll 1)$, one gets

$$
\mathbb{E}\left\{\phi^{2} / 2\right\} \simeq-p \lambda_{k}<\left(t_{2}-t_{1}\right)>_{2}+p^{2} \frac{\lambda_{k}^{2}}{2}<\left(t_{2}-t_{1}\right)^{2}>_{2}+\ldots
$$

In this series expansion, there is no divergent term, and the $p^{3 / 2}$ correction disappears. 
arbitrary temporal ${ }^{13}$ and spatial profiles of the magnetic field (see discussion in subsection IV.E).

\section{Specific temporal profiles}

For the Stejskal-Tanner temporal profile $f(t)$ shown in Fig. 2(c) (two rectangular pulses of duration $\delta$ ), the $f$ weighted time average of the function $\left(t_{2}-t_{1}\right)^{\alpha}$ for any positive power $\alpha$ can be found explicitly:

$$
\begin{aligned}
& <\left(t_{2}-t_{1}\right)^{\alpha}>_{2}= \\
& \frac{2 \delta^{\alpha+2}+2(1 / 2)^{\alpha+2}-(1 / 2+\delta)^{\alpha+2}-(1 / 2-\delta)^{\alpha+2}}{(\alpha+1)(\alpha+2)} .
\end{aligned}
$$

As an example, for a steady profile $(\delta=1 / 2$, Fig. $2(\mathrm{a}))$, the above relation is reduced to

$$
<\left(t_{2}-t_{1}\right)^{\alpha}>_{2}=\frac{2^{-\alpha}-1}{(\alpha+1)(\alpha+2)} .
$$

The other case of particular interest is the narrow-pulse approximation $(\delta \rightarrow 0)$, for which one derives for $\alpha>0$

$$
<\left(t_{2}-t_{1}\right)^{\alpha}>_{2} \simeq-(1 / 2)^{\alpha} \delta^{2} .
$$

The analytical computation of the $f$-weighted time average of the function $\left(t_{2}-t_{1}\right)^{\alpha}$ for arbitrary $\alpha$ is more difficult for other profiles, but its numerical realization is simple and straightforward. The most important case $\alpha=1$ can be still proceeded theoretically. For instance, one retrieves a classical expression for the trapezoidal Stejskal-Tanner profile shown in Fig. 2(d):

$<\left(t_{1}-t_{2}\right)>_{2}=\frac{1}{2} \tau^{2}+\tau \delta+\frac{1}{2} \delta^{2}-\frac{7}{15} \tau^{3}-\frac{7}{6} \tau^{2} \delta-\tau \delta^{2}-\frac{1}{3} \delta^{3}$.

If the ramp time $\tau$ is equal to 0 , the trapezoidal profile is replaced by the rectangular one, while the above relation is reduced to (74).

For a CPMG sequence, the $180^{\circ} \mathrm{RF}$ pulse is repeatedly applied to generate a train of spin echoes. The corresponding effective temporal profile $f(t)$ is a periodic repetition of a chosen shape $f_{0}(t)$ (e.g., rectangular pulse) and its inversion [see Fig. 2(b)]. The representation (80) allows one to show that the $f$-weighted time average $\left\langle\left(t_{1}-t_{2}\right)\right\rangle$, for a CPMG profile $f(t)$ with $k$ echoes is $k^{2}$ times smaller than the $f_{0}$-weighted time average $\left\langle\left(t_{1}-t_{2}\right)\right\rangle_{2}$. For example, if one repeats $k$ times the rectangular Stejskal-Tanner profile shown in Fig. 2(c), the corresponding time average for such CPMG sequence will be

$$
<\left(t_{1}-t_{2}\right)>_{2}=\frac{\delta^{2}(1 / 2-\delta / 3)}{k^{2}} .
$$

13 A similar kind of time dependence in the case of multiple-pulse PGSE diffusion measurements was proposed by Fordham et al. (1996).
For $\delta=1 / 2$, one retrieves the Carr-Purcell relation (4).

Finally, a simple calculation of the $f$-weighted time average $\left\langle\left(t_{1}-t_{2}\right)\right\rangle_{2}$ for sinusoidal and cosinusoidal profiles $f(t)=\sin (2 \pi k t)$ and $f(t)=\cos (2 \pi k t)$ gives $3 /\left(8 \pi^{2} k^{2}\right)$ and $1 /\left(8 \pi^{2} k^{2}\right)$ respectively.

\section{E. Discussion on the correction term}

As we mentioned above, the expression (90) is an extension of the results by de Swiet and Sen (1994); Mitra et al. (1992, 1993); Sen et al. (1994). To compare the two approaches, let us consider the restricted diffusion under a linear magnetic field gradient, for which the expression (90) becomes

$$
\mathbb{E}\left\{\frac{\phi^{2}}{2}\right\} \simeq p<\left(t_{1}-t_{2}\right)>_{2}\left(1-\mu_{2} p^{1 / 2} \frac{<\left(t_{2}-t_{1}\right)^{3 / 2}>_{2}}{<\left(t_{2}-t_{1}\right)>_{2}}\right) .
$$

Two particular choices of the temporal profiles were discussed in the literature.

In the narrow-pulse approximation, one uses Eq. (94) to write

$$
\mathbb{E}\left\{\frac{\phi^{2}}{2}\right\} \simeq(p / 2) \delta^{2}\left(1-\mu_{2}(p / 2)^{1 / 2}\right) .
$$

Using the value $\mu_{2}=8 /(3 \sqrt{\pi})$ for the slab geometry, one finally obtains

$$
\mathbb{E}\left\{\frac{\phi^{2}}{2}\right\} \simeq(p / 2) \delta^{2}\left(1-\frac{4}{3 \sqrt{\pi}} \sqrt{D T / 2} \frac{2}{L}\right),
$$

where the definition of $p^{1 / 2}$ was explicitly used. In this relation, the factor $2 / L$ can be associated with the surfaceto-volume ratio of a slab. For a cylinder and a sphere, the value of $\mu_{2}$ is twice smaller than for the slab (see Table II), so that the last factor would be $1 / L$, where $L$ is the radius. An elementary calculation shows that $1 / L$ is equal to $S /(V d)$ for both cases, where $d$ is the dimension of space. Consequently, the above relation can be written in a unique form for three basic domains as

$$
\mathbb{E}\left\{\frac{\phi^{2}}{2}\right\} \simeq(p / 2) \delta^{2}\left(1-\frac{4}{3 \sqrt{\pi}} \sqrt{D T / 2} \frac{S}{V d}\right) .
$$

This result was derived by Mitra et al. (1992) using the properties of Green functions near a flat reflecting boundary. The relation (100) was argued to be valid for any geometry, providing a way to measure the surface-tovolume ratio of the confining medium.

A steady magnetic field gradient is another commonly considered choice of the temporal profile. In this case, one finds

$<\left(t_{1}-t_{2}\right)>_{2}=\frac{1}{12} \quad \frac{<\left(t_{2}-t_{1}\right)^{3 / 2}>_{2}}{<\left(t_{2}-t_{1}\right)>_{2}}=\frac{12(4-\sqrt{2})}{35}$. 
For a sphere, the relation (97) can be reduced to

$$
\mathbb{E}\left\{\frac{\phi^{2}}{2}\right\} \simeq \frac{p}{12}\left(1-\sqrt{D T / 2} \frac{32(2 \sqrt{2}-1)}{105 \sqrt{\pi}} \frac{S}{V}\right),
$$

where $3 / L$ was replaced by the surface-to-volume ratio of a sphere. One retrieves the result by de Swiet and Sen (1994), which was argued to be valid for any statistically isotropic confining medium. A similar expression can be obtained for a slab geometry,

$$
\mathbb{E}\left\{\frac{\phi^{2}}{2}\right\} \simeq \frac{p}{12}\left(1-3 \sqrt{D T / 2} \frac{32(2 \sqrt{2}-1)}{105 \sqrt{\pi}} \frac{2}{L}\right),
$$

where the factor $2 / L$ can be associated with the surfaceto-volume ratio of a slab. Since this geometry is not isotropic, the relation (102) could be applied only after averaging over all spatial orientations of the confining domain. This operation would suppress an additional prefactor 3 appeared in (103). If one considers a fixed slab, the spatial orientation is not applicable, and the prefactor 3 should be taken into account. The case of a cylinder can be similarly treated.

Although the shapes of the basic domains are different, the leading and correction terms are the same for all three cases. This is a characteristic feature of the slow diffusion regime when the signal attenuation is essentially independent of the particular geometry of the confining domain. One can expect that a similar relation will hold for more realistic structures in porous material or biological tissues. Numerical studies of more complicated domains would be useful to clarify this point.

The dependence of the correction term on the surfaceto-volume ratio was considered by Mitra et al. as a way to determine this important characteristic of porous materials in a NMR experiment. In this perspective, the extension (90) to arbitrary temporal profile $f(t)$ of the magnetic field becomes much more valuable. On the one hand, it allows one to calculate this correction for specific gradient profiles used in experiment. On the other hand, one can think to optimize the temporal profile $f(t)$ in such a way that it enhances the contribution of the correction term so that it may facilitate the determination of the surface-to-volume ratio of a studied porous material.

At the same time, we should stress the ambiguity of this notion in the present context. The surface-to-volume ratio naturally appears in the relation (103) as the fraction of spins in the neighbourhood of the interface, within the diffusion length $\sqrt{D T}$, with respect to the total number of spins (proportional to the volume). One may wonder, however, whether the whole surface does contribute in the same way. To illustrate this point, let us consider restricted diffusion in a parallelepiped of size $L \times H \times H$. If the magnetic field gradient is applied along the $x$ axis, the presence of restrictive walls along the $y$ and $z$ directions does not change the signal attenuation. In other words, the above analysis for the slab is still valid in this case. This is a simple consequence of the fact that the three components (or coordinates) of the Brownian motion with normal reflections on the boundary are independent in this geometry. One thus gets the relation (103) with ratio $2 / L$. However, the surface-to-volume ratio for the parallelepiped is $\left(2 H^{2}+4 H L\right) /\left(H^{2} L\right)=2 / L+4 / H$. By varying the dimension $H$ of this domain, one can formally produce ratios between $2 / L$ and infinity. This simple example clearly shows that various regions of the surface may contribute in different ways: two planes orthogonal to the gradient direction give $2 / L$, while the other four planes do not contribute at all. In general, one may expect that the contribution of each boundary point would be weighted by projection of the gradient direction onto the normal vector at this point. To account for this effect, the orientation of the confining domain can be averaged over all spatial directions, which gave the supplementary prefactor $1 / 3$. If the parallelepiped is replaced by a cube with $H=L$, the surface-to-volume ratio is equal to $6 / L$, and one retrieves $2 / L$ after "orientational average". Such a trick, even when possible, is limited to spatially isotropic structures. In our opinion, a more profound analysis of this problem is required, especially in view of applications to realistic media. The MCF approach appears to be an efficient theoretical and numerical tool to investigate the correction terms for different confining geometries.

\section{MOTIONAL NARROWING REGIME $(p \gg 1)$}

When the diffusion length $\sqrt{D T}$ strongly exceeds the characteristic dimension $L(p \gg 1)$, the diffusing spins explore the whole domain a number of times during their motion. ${ }^{14}$ This is the so-called "motional narrowing or motionally averaging regime" for which the geometrical properties of the confining domain are important. In particular, the specific signal attenuation in this regime is at the origin of the edge enhancement in NMR microscopy (Callaghan et al., 1993; Callaghan, 1995; Hyslop and Lauterbur, 1991; Putz et al., 1992). In this section, we first obtain the leading terms and then discuss their corrections.

\footnotetext{
14 To avoid a possible ambiguity, we stress again that the confining domain is considered here to be bounded. This situation is significantly different with respect to a commonly used model of porous structure, e.g., in rocks (Hürlimann et al., 1994; Latour et al., 1995). In the latter case, the diffusion of nuclei is restricted within small cavities that are interconnected between them forming an infinite (or very big) pore network. In the long time limit, the nuclei can travel between several pores, but they never explore the whole structure. The results of this section are not applicable to such a pore network.
} 


\section{A. Leading terms}

As for the slow diffusion regime, we are going first to calculate the leading term of the second moment:

$$
\mathbb{E}\left\{\frac{\phi^{2}}{2}\right\}=\sum_{m=0}^{\infty} \mathcal{B}_{0, m}<e^{-p\left(t_{2}-t_{1}\right) \lambda_{m}}>_{2} \mathcal{B}_{m, 0} .
$$

In the limit $p \rightarrow \infty$, the above exponential function converges to a delta-function so, in a first approximation, it can be replaced by $\left(p \lambda_{m}\right)^{-1} \delta\left(t_{2}-t_{1}\right)$. One gets

$$
\mathbb{E}\left\{\frac{\phi^{2}}{2}\right\} \simeq p^{-1}<\delta\left(t_{2}-t_{1}\right)>_{2} \sum_{m=1}^{\infty} \mathcal{B}_{0, m} \lambda_{m}^{-1} \mathcal{B}_{m, 0}
$$

where the constant term (for $m=0$ ) vanished due to the rephasing condition. The $f$-weighted time average of the delta-function is simply

$$
<\delta\left(t_{2}-t_{1}\right)>_{2}=\int_{0}^{1} d t f^{2}(t)
$$

The sum over $m$, denoted as $\zeta_{-1}$ according to (67), depends on the confining geometry and the magnetic field spatial profile $B(\mathbf{r})$. In the next subsection, this constant will be calculated explicitly for basic confining domains and different spatial profiles.

The leading term of the second moment in the motional narrowing regime is then

$$
\mathbb{E}\left\{\frac{\phi^{2}}{2}\right\} \simeq p^{-1} \zeta_{-1} \int_{0}^{1} d t f^{2}(t)
$$

In a similar way, one can compute the leading terms for higher order moments. An example of such calculation for the fourth moment is given in Appendix $\mathrm{C}$ :

$$
\mathbb{E}\left\{\frac{\phi^{4}}{4 !}\right\} \simeq p^{-2} \zeta_{-1}^{2} \frac{1}{2}\left(\int_{0}^{1} d t f^{2}(t)\right)^{2} .
$$

One can guess their general form for even orders:

$$
\mathbb{E}\left\{\frac{\phi^{2 n}}{(2 n) !}\right\} \simeq p^{-n} \zeta_{-1}^{n} \frac{1}{n !}\left(\int_{0}^{1} d t f^{2}(t)\right)^{n},
$$

while a systematic computational technique is required for a rigorous demonstration of this relation. Bringing together the leading terms of even moments, one derives an extension of the classical result for the motional narrowing regime:

$$
E \simeq \exp \left[-\frac{q^{2}}{p} \zeta_{-1} \int_{0}^{1} d t f^{2}(t)\right] .
$$

The dependence of the $\ln E$ as a function of $q^{2} / p$ was first outlined by Robertson (1966) for the one-dimensional diffusion under steady linear gradient, and then extended to the case of a cylinder and a sphere by Neuman (1974). In fact, the Neuman's approach might be directly extended to any confining domain. At the same time, it was not appropriate to integrate arbitrary temporal or spatial profiles of the magnetic field.

\section{B. Corrections to the leading term}

While the leading asymptotic behavior in Eqs. (107) and (109) is general for any temporal profile $f(t)$, the correction terms strongly depend on the particular choice of the function $f(t)$. To illustrate this point, we shall calculate the correction to the leading term of the second moment for two typical profiles used in NMR experiments.

\section{Stejskal-Tanner temporal profile}

Once the temporal profile $f(t)$ is chosen, the $f$ weighted time average of the exponential function in (104) can be calculated explicitly. In particular, for the two rectangular pulses shown in Fig. 2(c), one gets

$$
\begin{aligned}
& \left.<e^{-p \lambda_{m}\left(t_{2}-t_{1}\right)}\right\rangle_{2}=\frac{2 \delta}{p \lambda_{m}}- \\
& \frac{2+e^{-p \lambda_{m}(1 / 2+\delta)}+e^{-p \lambda_{m}(1 / 2-\delta)}-2 e^{-p \lambda_{m} / 2}-2 e^{-p \lambda_{m} \delta}}{p^{2} \lambda_{m}^{2}} .
\end{aligned}
$$

The right hand side of this relation was proposed by Murday and Cotts (1968) to calculate the self-diffusion coefficient of a confined liquid lithium within a spherical restriction.

The spatial average of this function with $\mathcal{B}_{0, m} \mathcal{B}_{m, 0}$ (summation over $m$ from 1 to infinity) gives the second moment $\mathbb{E}\left\{\phi^{2} / 2\right\}$. If $p$ is big enough, the exponential functions in (111) rapidly vanish since the eigenvalues $\lambda_{m}$ progressively increase. For $\delta<1 / 2$, one obtains the second moment to a very good approximation:

$$
\mathbb{E}\left\{\frac{\phi^{2}}{2}\right\} \simeq \zeta_{-1}(2 \delta) p^{-1}-2 \zeta_{-2} p^{-2},
$$

where both coefficients $\zeta_{-1}$ and $\zeta_{-2}$ are defined by (67). In addition, one can recognize the factor $2 \delta$ as the integral of the squared temporal profile (52):

$$
\int_{0}^{1} d t f^{2}(t)=2 \delta
$$

One sees that, for the second moment in the motional narrowing regime with the Stejskal-Tanner temporal profile (52), all the possible complexity of the confining geometry and magnetic field spatial distribution is represented via the two constants $\zeta_{-1}$ and $\zeta_{-2}$ only. Moreover, 
if $q$ is small or $p$ is relatively large, the second moment gives the most significant contribution to the signal, so that one can use the first order approximation:

$$
E \simeq \exp \left[-q^{2}\left(\zeta_{-1}(2 \delta) p^{-1}-2 \zeta_{-2} p^{-2}\right)\right] .
$$

For the particular case of a steady profile $(\delta=1 / 2)$, the exponential function $e^{-p \lambda_{m}(1 / 2-\delta)}$ in (111) is constant, and one has

$\mathbb{E}\left\{\frac{\phi^{2}}{2}\right\}=\sum_{m=1}^{\infty} \mathcal{B}_{0, m} \mathcal{B}_{m, 0}\left(\frac{1}{p \lambda_{m}}-\frac{e^{-p \lambda_{m}}-4 e^{-\frac{1}{2} p \lambda_{m}}+3}{p^{2} \lambda_{m}^{2}}\right)$.

This very same structure of the second moment was given by Robertson [compare to Eq. (16) taking $\lambda_{m}$ and $\mathcal{B}_{0, m}$ from Eqs. (57),(59)], and then reproduced by different authors. For large enough $p$, one gets

$$
\mathbb{E}\left\{\frac{\phi^{2}}{2}\right\} \simeq \zeta_{-1} p^{-1}-3 \zeta_{-2} p^{-2}
$$

In this case, the integral of the squared temporal profile is equal to 1 . The comparison between this asymptotic result and the precise computation of the second moment is shown in Fig. 4. One can notice that the relation (116) is applicable even for $p$ around 1, where the motional narrowing regime was not expected to be valid.

Interestingly, the relation (115) can be already recognized in the paper of Tarczon and Halperin (1985) where there was no sign of the Laplace operator eigenbasis. In that work, the restricted diffusion on an interval was considered in the case of a steady magnetic field of arbitrary spatial profile that was periodically extended over the whole coordinate axis. The elements $\mathcal{B}_{0, m}$ then appeared as Fourier coefficients of the spatial profile $B(\mathbf{r})$, while the time average (111) was derived by a specific and cumbersome technique. Similarly, the relation (72) for $\zeta_{1}$ was also found in the one-dimensional case by Tarczon and Halperin (1985).

Both coefficients $\zeta_{-1}$ and $\zeta_{-2}$ in the case of a linear magnetic field gradient were found by Robertson (1966) for a slab and by Neuman (1974) for a cylinder and a sphere. Their values are reproduced in Table III. ${ }^{15}$ The calculation for the parabolic magnetic field (55) can be done in a similar way. In the case of a slab geometry, one uses the explicit formulae (57), (60) for $\lambda_{m}$ and $\mathcal{B}_{0, m}$ to get:

$$
\begin{aligned}
& \zeta_{-1}=\frac{8}{\pi^{6}} \sum_{m=1}^{\infty} \frac{1}{m^{6}}=\frac{8}{945}, \\
& \zeta_{-2}=\frac{8}{\pi^{8}} \sum_{m=1}^{\infty} \frac{1}{m^{8}}=\frac{4}{4725} .
\end{aligned}
$$

15 Note a typographical error in (Neuman, 1974): for the cylinder, the coefficient $\zeta_{-1}$ was misprinted as $7 / 296$ instead of $7 / 96$.

\begin{tabular}{|c|c|c|c|c|}
\hline & magnetic field & slab & cylinder & sphere \\
\hline$\zeta_{1}$ & & 1 & 1 & 1 \\
$\zeta_{0}$ & & $1 / 12$ & $1 / 4$ & $1 / 5$ \\
$\tilde{\zeta}_{0}$ & linear & $1 / 3$ & $1 / 4$ & $1 / 5$ \\
$\zeta_{-1}$ & & $1 / 120$ & $7 / 96$ & $8 / 175$ \\
$\zeta_{-2}$ & & $17 / 20160$ & $11 / 512$ & $83 / 7875$ \\
\hline$\zeta_{1}$ & & $4 / 3$ & 2 & $12 / 5$ \\
$\zeta_{0}$ & & $4 / 45$ & $1 / 12$ & $12 / 175$ \\
$\tilde{\zeta}_{0}$ & parabolic & $1 / 5$ & $1 / 3$ & $3 / 7$ \\
$\zeta_{-1}$ & & $8 / 945$ & $1 / 192$ & $8 / 2625$ \\
$\zeta_{-2}$ & & $4 / 4725$ & $1 / 2880$ & $148 / 1010625$ \\
\hline
\end{tabular}

TABLE III Several coefficients $\zeta_{k}$ for a slab, a cylinder and a sphere under linear gradient and parabolic magnetic fields. Coefficients $\tilde{\zeta}_{0}$ and $\zeta_{1}$ are directly obtained by Eqs. (70), (72). The values of $\zeta_{-1}$ and $\zeta_{-2}$ for a slab, a cylinder and a sphere were calculated by Robertson and Neuman for a magnetic field with a linear gradient profile. The case of parabolic magnetic field is considered in Appendix D.

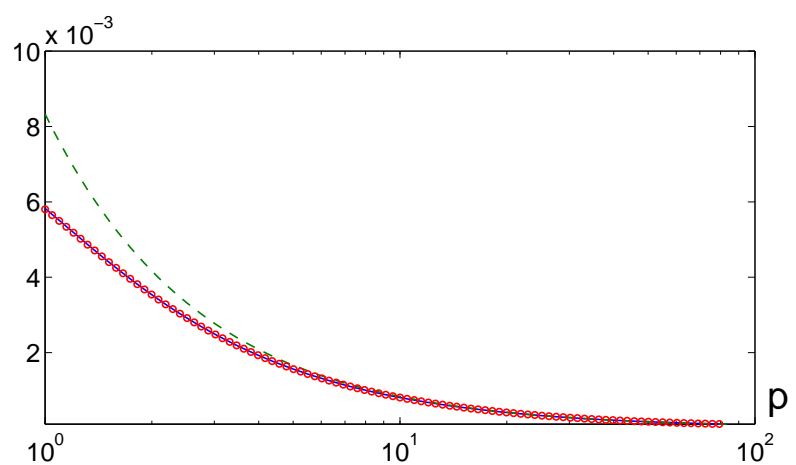

FIG. 4 The second moment $\mathbb{E}\left\{\phi^{2} / 2\right\}$ (solid line) as a function of $p$ for the restricted diffusion between two parallel planes (in a slab) under steady linear magnetic field gradient. For large $p$, the second moment is compared to its leading term in the motional narrowing regime with (circles) and without (dashed line) correction term in (116).

Note that the above value of the coefficient $\zeta_{-1}$ for the parabolic magnetic field was found by Zielinski and Sen (2000). A more complicated derivation for a cylinder and a sphere is briefly presented in Appendix D (the found values are resumed in Table III).

\section{Oscillating temporal profile}

In the case of a sinusoidal temporal profile $f(t)=$ $\sin (2 \pi k t)$, one gets

$$
\begin{aligned}
<e^{-p \lambda_{m}\left(t_{2}-t_{1}\right)}>_{2}= & \frac{1}{2} \frac{p^{3} \lambda_{m}^{3}+4 \pi^{2} k^{2} p \lambda_{m}+8 \pi^{2} k^{2}}{\left(p^{2} \lambda_{m}^{2}+4 \pi^{2} k^{2}\right)^{2}} \\
& -\frac{4 \pi^{2} k^{2} e^{-p \lambda_{m}}}{\left(p^{2} \lambda_{m}^{2}+4 \pi^{2} k^{2}\right)^{2}}
\end{aligned}
$$


The second term vanishes rapidly in the limit $p \rightarrow \infty$, while the first term can be expanded into the series in powers of $p^{-1}$ :

$<e^{-p \lambda_{m}\left(t_{2}-t_{1}\right)}>_{2}=\frac{1}{2} p^{-1} \lambda_{m}^{-1}-2 \pi^{2} k^{2} p^{-3} \lambda_{m}^{-3}+O\left(p^{-4}\right)$.

The prefactor $1 / 2$ in front of $p^{-1}$ is indeed equal to the integral of the squared temporal profile $f(t)$ as required. In contrast to the case of the Stejskal-Tanner profile, the correction terms start from $p^{-3}$ and contain higher orders in a series expansion.

A similar analysis for a cosinusoidal temporal profile $f(t)=\cos (2 \pi k t)$ leads to a different result:

$$
\begin{aligned}
<e^{-p \lambda_{m}\left(t_{2}-t_{1}\right)}>_{2}= & \frac{1}{2} \frac{p \lambda_{m}\left(p^{2} \lambda_{m}^{2}-2 p \lambda_{m}+4 \pi^{2} k^{2}\right)}{\left(p^{2} \lambda_{m}^{2}+4 \pi^{2} k^{2}\right)^{2}} \\
& -\frac{p^{2} e^{-p \lambda_{m}}}{\left(p^{2} \lambda_{m}^{2}+4 \pi^{2} k^{2}\right)^{2}} .
\end{aligned}
$$

In the limit $p \rightarrow \infty$, one gets

$$
\begin{aligned}
\left\langle e^{-p \lambda_{m}\left(t_{2}-t_{1}\right)}\right\rangle_{2}= & \frac{1}{2} p^{-1} \lambda_{m}^{-1}-p^{-2} \lambda_{m}^{-2}- \\
& -2 \pi^{2} k^{2} p^{-3} \lambda_{m}^{-3}+O\left(p^{-4}\right),
\end{aligned}
$$

where the first correction to the leading term $p^{-1}$ is of order $p^{-2}$. While the leading term is always of order $1 / p$, independently of the temporal profile, the form of correction terms strongly depends on a particular choice of $f(t)$.

\section{DISCUSSION}

In the two previous sections, we have applied the MCF approach to retrieve, extend and reinterpret some classical results on diffusive NMR phenomena. After a sketch on the numerical implementation of this approach, we are going to discuss some open or poorly understood issues which can now be better investigated.

\section{A. Numerical implementation}

The implementation of the MCF approach for a numerical analysis is straightforward and simple, especially for the "basic" domains: a slab, a cylinder and a sphere. The matrices $\mathcal{B}$ and $\Lambda$, entirely determining all the moments $\mathbb{E}\left\{\phi^{n}\right\}$, have to be computed numerically only once for a chosen confining geometry and spatial profile of the magnetic field. The signal is then found through the series expansion (46).

Interestingly, the numerical computation of the signal can be performed in a much simpler way. The technique that we are going to resume was developed by Robertson and further extended by Barzykin $(1998,1999)$ to study the restricted diffusion under linear gradient magnetic field. To illustrate the idea, let us consider the BlochTorrey equation (9) with time-independent magnetic field $\beta B(\mathbf{r})$ :

$$
\left(\frac{\partial}{\partial t}-D \Delta+i \gamma \beta B(\mathbf{r})\right) \mathfrak{m}(\mathbf{r}, t)=0 .
$$

In general, the Fourier boundary condition (10) is imposed. The magnetization $\mathfrak{m}(\mathbf{r}, t)$ can be expanded over the Laplace operator eigenbasis:

$$
\mathfrak{m}(\mathbf{r}, t)=\sum_{m^{\prime}=0}^{\infty} c_{m^{\prime}}(t) u_{m^{\prime}}(\mathbf{r})
$$

The macroscopic signal $E$ would be obtained by integrating $\mathfrak{m}(\mathbf{r}, T)$ over the domain $\Omega$ :

$$
E=\sum_{m^{\prime}=0}^{\infty} c_{m^{\prime}}(T) \int_{\Omega} d \mathbf{r} u_{m^{\prime}}(\mathbf{r})=V^{1 / 2} \sum_{m^{\prime}=0}^{\infty} c_{m^{\prime}}(T) \tilde{U}_{m^{\prime}}
$$

where the new infinite-dimension vector $\tilde{U}$ is explicitly introduced:

$$
\tilde{U}_{m^{\prime}}=V^{-1 / 2} \int_{\Omega} d \mathbf{r} u_{m^{\prime}}(\mathbf{r})
$$

To find the coefficients $c_{m^{\prime}}(T)$, one substitutes the expansion (123) into the Bloch-Torrey equation (122), multiplies it by the eigenfunction $u_{m}^{*}(\mathbf{r})$ and integrates over $\Omega$. These operations lead to a set of ordinary differential equations:

$$
\frac{d}{d t} c_{m}(t)+\frac{D \lambda_{m}}{L^{2}} c_{m}(t)+i \gamma \beta \sum_{m^{\prime}=0}^{\infty} \mathcal{B}_{m, m^{\prime}} c_{m^{\prime}}(t)=0
$$

where the matrix $\mathcal{B}$ is defined by (39). The initial condition $\mathfrak{m}(\mathbf{r}, t=0)=\rho(\mathbf{r})$ implies that

$$
c_{m}(0)=\int_{\Omega} d \mathbf{r} \rho(\mathbf{r}) u_{m}^{*}(\mathbf{r})=V^{-1 / 2} U_{m}^{*},
$$

where the definition (31) of the vector $U$ was used.

The coefficients $c_{m}(t)$ can be thought as components of an infinite-dimension vector $C(t)$, and the above set of equations reads

$$
T \frac{d}{d t} C(t)=-(p \Lambda+i q \mathcal{B}) C(t)
$$

where the matrix $\Lambda$ is defined by (34). The solution of this ordinary differential equation is simply

$$
C(t)=\exp [-(p \Lambda+i q \mathcal{B}) t / T] C(0) .
$$

Bringing together the above relations, one can write the macroscopic signal in a compact form of a scalar prod- 
uct $^{16}$

$$
E=\left(\tilde{U} \cdot \exp [-(p \Lambda+i q \mathcal{B})] U^{*}\right)
$$

A particular simplification can be achieved for the reflecting boundary condition. Since the initial density is uniform, one gets $U_{m}=\tilde{U}_{m}=\delta_{m, 0}$. In this case, the macroscopic signal is simply equal to the first diagonal element of the exponential matrix:

$$
E=\left[e^{-p \Lambda-i q \mathcal{B}}\right]_{0,0}
$$

We stress that the relations (130) and (131) are exact for a time-independent magnetic field.

In fact, this result can be applied to numerically compute the signal for a given time-dependent profile $f(t)$. For this purpose, the interval $[0,1]$ is divided into a large number $K$ of subintervals of duration $\tau=1 / K$. On the $k^{t h}$ subinterval, the function $f(t)$ is approximated by a constant $f(k \tau)$. The signal can be numerically found with $^{17}$

$$
E \simeq\left(\tilde{U} \cdot\left(\prod_{k=0}^{K} \exp [-\tau(p \Lambda+i q f(k \tau) \mathcal{B})]\right) U^{*}\right)
$$

or, for the reflecting boundary condition, with

$$
E \simeq\left[\prod_{k=0}^{K} \exp [-\tau(p \Lambda+i q f(k \tau) \mathcal{B})]\right]_{0,0} .
$$

This is an extension of the Barzykin's numerical approach to an arbitrary spatial profile $B(\mathbf{r})$ of the magnetic field. The crucial point is that an unbounded increase of the eigenvalues $\lambda_{m}$ with $m$ allows one to truncate the infinitedimension matrices $\mathcal{B}$ and $\Lambda$ to moderate sizes (since the matrix $\Lambda$ stands as argument of the exponential function). Further computation of the matrix product in (132) or (133) with the help of commercial softwares like Matlab, Maple or Matematica is rapid and very accurate. This technique can be readily implemented for "basic" domains for which the matrices $\mathcal{B}$ and $\Lambda$ are explicitly

16 The surface relaxation attenuates the signal even in the case when no diffusion-sensitizing magnetic field is applied $(q=0)$. It is then convenient to normalize the signal with $q>0$ by the signal at $q=0$,

$$
E_{\text {norm }}=\frac{\left(\tilde{U} \cdot \exp [-(p \Lambda+i q \mathcal{B})] U^{*}\right)}{\left(\tilde{U} \cdot \exp [-p \Lambda] U^{*}\right)} .
$$

This normalization is not needed for the reflecting boundary condition, for which $\left(\tilde{U} \cdot \exp [-p \Lambda] U^{*}\right)=1$.

17 It is worth to note that the matrices $\mathcal{B}$ and $\Lambda$ do not commute, so that the product in (132) cannot be "reduced" to

$$
\exp \left[-\tau \sum_{k=0}^{K}(p \Lambda+i q f(k \tau) \mathcal{B})\right] \text {. }
$$

known. When the geometry of the confining domain $\Omega$ is more complicated, one needs first to compute the eigenbasis of the Laplace operator in order to build $\mathcal{B}$ and $\Lambda$. This is a classical problem in applied mathematics for which a number of efficient numerical algorithms have been developed. As for the "basic" domains, one needs only a small number of eigenmodes.

Note that the general matrix representation (51) of the moments $\mathbb{E}\left\{\phi^{n}\right\}$ could be derived from the expression (133) in the limit $\tau \rightarrow 0$ and $K \rightarrow \infty$. The main steps of such a derivation for a similar case of the multiple propagator approach are shown in Appendix E.

\section{B. Odd order moments}

The series expansion (46) of the signal $E$ involves, in general, both even and odd moments $\mathbb{E}\left\{\phi^{n}\right\}$ of the random phase $\phi$. In the literature, the odd moments forming the imaginary part of the signal are somehow missed: some authors proposed heuristic arguments or specific assumptions to neglect them (e.g., inversion symmetry of the confining domain), the others passed over in silence. In this subsection, we would like to discuss why the odd moments do not contribute, at least for typical cases considered in the literature. At the same time, several counter-examples will be given to illustrate a possible deviation from this common belief.

\section{First moment}

Although the results of this subsection could be derived directly from Eq. (51), we would like first to give an intuitive feeling for the reason why odd moments may or may not disappear under certain conditions. By definition, the first moment $\mathbb{E}\{\phi\}$ is equal to the $f$-weighted time average of the expectation $\mathbb{E}\left\{B\left(X_{t}\right)\right\}$. Here the magnetic field spatial profile $B(\mathbf{r})$ is averaged over all possible trajectories of the reflected Brownian motion started from uniformly distributed initial position (the density $\rho(\mathbf{r})$ is typically uniform for reflecting boundaries). In other words, the expectation $\mathbb{E}\left\{B\left(X_{t}\right)\right\}$ can be written as

$$
\mathbb{E}\left\{B\left(X_{t}\right)\right\}=\frac{1}{V} \int_{\Omega} d \mathbf{r} v(\mathbf{r}, t),
$$

where $v(\mathbf{r}, t)$ is the expectation for the reflected Brownian motion started from a fixed point $\mathbf{r}$ :

$$
v(\mathbf{r}, t)=\mathbb{E}\left\{B\left(X_{t}\right) \mid X_{0}=\mathbf{r}\right\}
$$

Note that this function is a solution of the diffusion equation

$$
\left(\frac{\partial}{\partial t}-D \Delta\right) v(\mathbf{r}, t)=0
$$


with the Neumann boundary condition and $v(\mathbf{r}, t=0)=$ $B(\mathbf{r})$. Consequently, the function $v(\mathbf{r}, t)$ can be interpreted as a "density of pseudo-particles" diffusing in domain $\Omega$ with reflecting boundary. The magnetic field $B(\mathbf{r})$ formally appears as the "initial density of pseudoparticles", while the expectation $\mathbb{E}\left\{B\left(X_{t}\right)\right\}$ gives the "total number of pseudo-particles" at time $t$. Note that this is only a formal illustration since the magnetic field $B(\mathbf{r})$ can be negative. Since the boundary is reflecting, there is no loss of these "pseudo-particles", and $\mathbb{E}\left\{B\left(X_{t}\right)\right\}$ is constant for any $t$ :

$$
\mathbb{E}\left\{B\left(X_{t}\right)\right\}=\mathbb{E}\left\{B\left(X_{0}\right)\right\}=\frac{1}{V} \int_{\Omega} d \mathbf{r} B(\mathbf{r}) .
$$

This result could be derived directly from Eq. (51) for $n=1$ :

$$
\mathbb{E}\left\{B\left(X_{t}\right)\right\}=[\mathcal{B}]_{0,0}=\frac{1}{V} \int_{\Omega} d \mathbf{r} B(\mathbf{r}) .
$$

From this point, different situations can be considered:

1. If the magnetic field $B(\mathbf{r})$ is such that its integral over domain $\Omega$ is equal to 0 , then the first moment $\mathbb{E}\{\phi\}$ is zero for any temporal profile $f(t)$. This is the case for a linear gradient $B(\mathbf{r})=r \cos \theta$ in a cylinder, a sphere, and a centered interval $(-1,1)$. However, this condition is limited to symmetric domains and linear gradients. For example, it is impossible to satisfy neither for a linear gradient in a non-symmetric domain nor for a parabolic magnetic field.

2. If the temporal profile $f(t)$ satisfies the rephasing condition (36), the constant expectation $\mathbb{E}\left\{B\left(X_{t}\right)\right\}$ vanishes for any magnetic field spatial profile $B(\mathbf{r})$, leading to $\mathbb{E}\{\phi\}=0$.

3. If none of the above conditions is satisfied, the first moment $\mathbb{E}\{\phi\}$ is not zero, providing a nontrivial contribution to the imaginary part of the signal $E$.

An example of a nontrivial contribution from the first moment can be given if the boundary is relaxing. In this case, $\mathbb{E}\left\{B\left(X_{t}\right)\right\}$ can be still interpreted as the "total number of pseudo-particles" which, in turn, varies in time due to the surface relaxation. Except for very specific cases, its $f$-weighted time average is not zero and the first moment does contribute.

These examples show that the situation with odd moments is not so simple as commonly believed. Moreover, the above analysis cannot be directly applied to higher order moments. For instance, the zero integral of the magnetic field spatial profile $\left(\mathcal{B}_{0,0}=0\right)$ is not sufficient to cancel higher odd moments since $\mathcal{B}_{m, m}$ are not necessarily zero for $m>0$. On the other hand, their cancellation due to the rephasing condition (36) remains an open question. In the next subsection, we give the proof in one case. A more detailed analysis, which would clarify the role of these moments, is out of the scope of this paper.

\section{Antisymmetric temporal profiles}

The most interesting situation corresponds to a cancellation of odd moments for an arbitrary spatial profile of the magnetic field due the rephasing condition. However, its derivation is much more difficult and would require a more profound study of the $f$-weighted time averages. Here we focus on a specific case when the temporal profile is antisymmetric with respect to the point $1 / 2$, i.e.,

$$
f(1-t)=-f(t) .
$$

A simple algebraic proof is based on the approximate relation of the subsection VI.A. Indeed, let us separate the matrix product in Eq. (133) in two parts: the first $K / 2$ factors remain unchanged, while the next $K / 2$ factors are modified according to a simple matrix identity $X Y=\left(Y^{*} X^{*}\right)^{*}$ :

$E \simeq\left[\prod_{k=0}^{K / 2} e^{-\tau[p \Lambda+i q \mathcal{B} f(k \tau)]}\left(\prod_{k=0}^{K / 2} e^{-\tau[p \Lambda-i q \mathcal{B} f((K-k) \tau)]}\right)^{*}\right]_{0,0}$.

In the second product, one uses $f((K-k) \tau)=-f(k \tau)$ to obtain

$E \simeq\left[\left(\prod_{k=0}^{K / 2} e^{-\tau[p \Lambda+i q \mathcal{B} f(k \tau)]}\right)\left(\prod_{k=0}^{K / 2} e^{-\tau[p \Lambda+i q \mathcal{B} f(k \tau)]}\right)^{*}\right]_{0,0}$.

It is clear now that the matrix in big square brackets is real-valued as the product of two matrices, one of them being the complex conjugate of the other. Consequently, the imaginary part of the signal $E$ is zero, so that all odd moments vanish. To complete the derivation, one takes the limit $\tau \rightarrow 0$ (and $K \rightarrow \infty$ ).

Since the usual temporal profiles (Stejskal-Tanner, sinusoidal, etc.) satisfy the condition (139) or its variations, the problem of the odd moments in such typical cases is now solved. We conjecture that the rephasing condition (36) is sufficient for cancellation of the odd moments in general. Its mathematical demonstration remains an interesting open problem.

\section{Cumulant expansion}

The knowledge of the even moments of the random phase $\phi$ gives the macroscopic signal in the form of a series expansion (46). The basic properties of the exponential function allow one to find also the series representation of the logarithm of the signal which is called "cumulant expansion":

$$
\ln E=\sum_{n=1}^{\infty} \frac{\left(-q^{2}\right)^{n}}{(2 n) !} \prec \phi^{2 n} \succ_{c} .
$$

Here $\prec \phi^{2 n} \succ_{c}$ denote so-called "cumulants" or "cumulant moments" which can be expressed through the "or- 
dinary" moments, for example,

$\prec \phi^{2} \succ_{c}=\mathbb{E}\left\{\phi^{2}\right\}, \quad \prec \phi^{4} \succ_{c}=\mathbb{E}\left\{\phi^{4}\right\}-3\left(\mathbb{E}\left\{\phi^{2}\right\}\right)^{2}, \quad \ldots$

(under the condition that odd moments are zero). The characteristic feature of the cumulant expansion is that the fourth and higher order cumulant moments are exactly zero for a Gaussian phase $\phi$. In this case, the cumulant expansion is naturally truncated to the second moment, and one obviously retrieves the Gaussian form of the signal. If the phase is not Gaussian, the cumulant expansion gives the higher order corrections to the Gaussian behavior. The fourth and higher cumulant moments indicate to which extent the Gaussian phase approximation remains valid. For this reason, the cumulant expansion was preferred by some authors to the ordinary expansion. At the same time, it should be clear that both representations contain exactly the same information about the signal $E$.

In spite of its apparent convenience, the cumulant expansion has an important mathematical "defect". As was recently discussed by Frøhlich et al. (2006), the cumulant expansion has a limited convergence radius. It means that the series expansion (142) converges only inside a certain disk in the complex plane of values $q$. In other words, there exists a critical value $q_{c}$ such that the above relation is divergent for $q \geq q_{c}$ (see Frøhlich et al. (2006) for a more detailed discussion on this topic). At the same time, the "ordinary" expansion (46) is absolutely convergent for any value of $q$.

\section{Apparent diffusion coefficient}

Under weak diffusion-sensitizing magnetic field $(q \ll$ $1)$, the signal is mainly determined by the second moment $\mathbb{E}\left\{\phi^{2} / 2\right\}$, while the higher order moments can be neglected, i.e.,

$$
E \simeq 1-q^{2} \mathbb{E}\left\{\phi^{2} / 2\right\} \simeq \exp \left[-q^{2} \mathbb{E}\left\{\phi^{2} / 2\right\}\right]
$$

whatever the magnitude of the dimensionless diffusion coefficient $p$. In the limit $p \rightarrow 0$, the second moment $\mathbb{E}\left\{\phi^{2} / 2\right\}$ is proportional to $p$ according to (73), whence $\ln E \propto q^{2} p \propto D$. When $p$ increases, the second moment is not necessarily dominated by its leading term, and $\ln E$ is not proportional to the free diffusion coefficient $D$ any more. This deviation was experimentally observed by Woessner (1963) and traditionally characterized by apparent (or effective) diffusion coefficient $(A D C)$. In our notations, the ADC can be defined as ${ }^{18}$

$\frac{D_{a p p}}{D}=\mathbb{E}\left\{\phi^{2} / 2\right\}\left(p<\left(t_{1}-t_{2}\right)>_{2} \frac{L^{2}}{V} \int_{\Omega} d \mathbf{r}|\nabla B(\mathbf{r})|^{2}\right)^{-1}$

The ratio $D / D_{a p p}$ is called "tortuosity" and considered as important macroscopic characteristics of porous materials or biological tissues (Helmer et al., 1995,b; Latour et al., 1993; Mair et al., 1999; Mitra et al., 1992, 1993). It shows how the diffusion of spins is "slowed down" by the presence of restrictive boundaries. In other words, the unrestricted diffusion of spins with the apparent diffusion coefficient $D_{a p p}$ is meant to represent the restricted diffusion of spins with their free diffusion coefficient $D$. Of course, this qualitative picture is just a simplified interpretation of restricted diffusion. Nonetheless, the apparent diffusion coefficient was used for a long time as a convenient characteristics for the confining geometry of porous materials or biological tissues. For example, human lung diseases like emphysema can be identified by an increase of the ADC due to enlargement or partial destruction of the alveolar tissue (Möller et al., 2002; Saam et al., 2000; van Beek et al., 2004; Yablonskiy et al., 2002). In spite of numerous applications of the ADC in different branches of the NMR industry, this notion remains limited to the first order approximation (144) of the general series expansion (46).

The apparent diffusion coefficient is directly related to another widely used concept of " $b$-value" or " $b$ coefficient". To find the ADC in experiment, one measures the macroscopic signal as a function of the parameters of the applied magnetic field (typically, gradient intensity $g$ or duration $T$ ). The substitution of the second moment, expressed from (144) as $\mathbb{E}\left\{\phi^{2} / 2\right\} \simeq-\ln E / q^{2}$,

18 We should note that this "NMR definition" is not similar to the "classical" definition, when time-dependent diffusion coefficient $D(t)$ appeared as a measure of the mean square displacements in time $t$,

$$
\mathbb{E}\left\{[\mathbf{r}(t)-\mathbf{r}(0)]^{2}\right\}=2 d D(t) t .
$$

As a "kinetic" characteristic of the reflected Brownian motion, $D(t)$ is totally independent of the applied magnetic field, while the NMR diffusion coefficient (ADC) is sensitive to its temporal and spatial profiles. In our notations, the "kinetic" diffusion coefficient can be written as a function of $p$ according to

$$
D(p)=D(2 p d)^{-1} \mathbb{E}\left\{\left[B\left(X_{1}\right)-B\left(X_{0}\right)\right]^{2}\right\},
$$

where $B(\mathbf{r})=\mathbf{r} / L$ is a formal notation. For reflecting boundary, one obtains a compact matrix form

$$
D(p)=D(p d)^{-1}\left[\mathcal{B}\left(I-e^{-p \Lambda}\right) \mathcal{B}\right]_{0,0} .
$$

In the limit $p \rightarrow 0$, one retrieves $D(p) \rightarrow D$ as required. A similar relation, written in a form of series expansion, can be recognized in (Mitra et al., 1993), where it was derived for a sphere with reflecting boundary. In the remainder of this review, we shall consider the NMR diffusion coefficient only. 
to (145) leads to a simple relation

$$
D_{a p p} \simeq \frac{-\ln E}{b},
$$

where the $b$-value is defined as

$$
b=\frac{q^{2} p}{D}<\left(t_{1}-t_{2}\right)>_{2} \frac{L^{2}}{V} \int_{\Omega} d \mathbf{r}|\nabla B(\mathbf{r})|^{2} .
$$

This is an extension of the classical definition, $b=$ $\gamma^{2} g^{2} T^{3} / 12$, to the case of arbitrary spatial and temporal profile of the magnetic field. The relation (146) is often written as

$$
E \simeq e^{-b D_{a p p}}
$$

While the ADC is intended to represent the effect of confining geometry, the $b$-value resumes all information about the applied magnetic field. The fact that a single parameter might be used to describe the whole experimental setup was widely employed in practical applications, particularly in medical imaging. For example, some authors gave $b$-value without even mentioning the gradient intensity, duration or temporal profile. It should be however clear that such a simplification is exaggerated. Since the diffusive NMR phenomena are governed by at least two independent parameters $p$ and $q$, the $b$ value alone cannot capture the whole picture. We stress that the $b$-value is only a useful notation for the combination of different parameters in Eq. (147). We shall illustrate this point by the following example.

For the slow diffusion regime $(p \ll 1)$, the second moment $\mathbb{E}\left\{\phi^{2} / 2\right\}$ is given by Eq. (90), whence one gets

$$
D_{a p p} \simeq D\left(1-\sqrt{p} \mu_{2} \frac{<\left(t_{2}-t_{1}\right)^{3 / 2}>_{2}}{<\left(t_{2}-t_{1}\right)>_{2}}\right),
$$

where a linear magnetic field gradient was considered. In particular, one retrieves the results by Mitra et al. (1992) and de Swiet and Sen (1994) for narrow-pulse and steady temporal profiles:

$$
\frac{D_{a p p}}{D} \simeq 1-\frac{4}{3 \sqrt{\pi}} \sqrt{D T / 2} \frac{S}{V d}
$$

and

$$
\frac{D_{a p p}}{D} \simeq 1-\frac{32(2 \sqrt{2}-1)}{105 \sqrt{\pi}} \sqrt{D T / 2} \frac{S}{V}
$$

respectively, where Eqs. (100, 102) were used.

The relation (149) does not depend at all on the dimensionless gradient intensity $q$. Consequently, the parameters $q$ and $p$ can be changed in such a way that $D_{a p p}$ would be substantially modified according to (149), while the $b$-value is kept fixed. This simple example shows that two experiments with the same $b$-value can give very different values of ADC. The knowledge of the $b$-value alone is not sufficient to compare different medical measurements presented in the literature (e.g., ADC maps of the lungs). At this point, we remind that the notion of $\mathrm{ADC}$ and relations (146), (148) are applicable only when the Gaussian phase approximation is valid.

In the motional narrowing regime $(p \gg 1)$, one gets the second moment from (107) so that

$$
D_{a p p} \simeq D\left(p_{0} / p\right)^{2},
$$

where

$$
p_{0}^{2}=\frac{\zeta_{-1}}{\left.<\left(t_{1}-t_{2}\right)\right\rangle_{2}} \int_{0}^{1} d t f^{2}(t) .
$$

The coefficient $p_{0}^{2}$ represents the relevant information about the temporal and spatial profiles of the magnetic field, as well as about the confining geometry. We propose a simple interpolation formula between very slow diffusion $\left(D_{a p p} \simeq D\right)$ and motional narrowing regimes:

$$
D_{a p p} \simeq D\left(1+\left(p / p_{0}\right)^{2}\right)^{-1} .
$$

Note that this relation does not account for the $p^{1 / 2}$ correction of the slow diffusion regime. As the notion of the apparent diffusion coefficient itself, this formula may be used only for the magnetic fields of relatively small intensity when the Gaussian form of the macroscopic signal is still valid.

Both theoretical relations (149) and (154) can be compared to the recent experimental measurement of the apparent diffusion coefficient by Hayden et al. (2004). In their setup, a cylindrical borosilicate glass cell of diameter $L=4.82 \mathrm{~cm}$ was filled with helium-3 gas $\left(\gamma \simeq 2.04 \cdot 10^{8} \mathrm{rad} \mathrm{T}^{-1} \mathrm{~s}^{-1}\right)$ to a pressure of 1 Torr. The rescaling of the experimental conditions (temperature and pressure) to a reference measurement gave the free diffusion coefficient $D \simeq 0.14 \mathrm{~m}^{2} / \mathrm{s}$ (Barbé et al., 1974; Bendt, 1958). The steady linear magnetic field gradient was applied in a perpendicular direction to the cylinder axis. The apparent diffusion coefficient $D_{a p p}$ was measured for different echo times $T$. We remind that, for this particular case, one has $\mu_{2}=4 /(3 \sqrt{\pi})$ and $\zeta_{-1}=7 / 96$, while the $f$-weighted time averages $\left\langle\left(t_{2}-t_{1}\right)\right\rangle_{2}$ and $<\left(t_{2}-t_{1}\right)^{3 / 2}>_{2}$ are given by (101) for the steady temporal profile. One then finds $p_{0}^{2}=7 / 8$.

The comparison between the experimental data and the above theoretical relations is shown in Fig. 5. For the slow diffusion regime (small $p$ ), the relation (149) remains in a very good agreement with experiment, until the correction term exceeds unity. From this point, the apparent diffusion coefficient is negative, and the slow diffusion regime becomes invalid. In the motional narrowing regime (large $p$ ), one observes the expected behavior $p^{-2}$. The use of the interpolation formula (154) allows one to avoid unphysical divergence when $p$ is getting smaller. Note that this formula gives good results even for very small $p$, when the motional narrowing regime is 


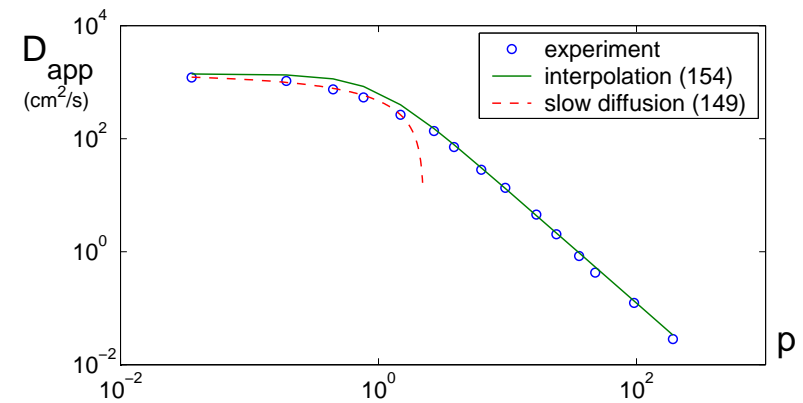

FIG. 5 Apparent diffusion coefficient as a function of $p$ for a cylindrical cell. The slow diffusion correction (149) and the interpolation formula (154) are compared to the spin-echo measurements in a borosilicate glass cell by Hayden et al. (2004). The shown experimental data have been communicated to the author by Dr. M. E. Hayden.

not formally applicable. In this region, the small deviation to the experimental data is related to the slow diffusion correction term which was not taken into account in Eq. (154). In summary, one can see that both theoretical relations are in good agreement with experimental measurements over four orders of magnitude. This result may look more exciting if one recalls that there are no adjustable parameters in the theoretical relations.

\section{E. Narrow-pulse approximation}

The Stejskal-Tanner profile (52) in the limit of vanishing pulse duration $\delta$, known as "narrow- (or short-) pulse approximation", has been applied for a long time to study restricted diffusion. ${ }^{19}$ Its main advantage is that diffusive motion during these short pulses can be neglected, so that the accumulated phase becomes

$$
\phi \simeq \delta B\left(X_{0}\right)-\delta B\left(X_{1 / 2}\right),
$$

and one gets the signal in a simple form

$$
E=\int_{\Omega} d \mathbf{r}_{0} \rho\left(\mathbf{r}_{0}\right) \int_{\Omega} d \mathbf{r} \exp \left[i q \delta\left(B\left(\mathbf{r}_{0}\right)-B(\mathbf{r})\right)\right] G_{T / 2}\left(\mathbf{r}_{0}, \mathbf{r}\right),
$$

where the time moment $T / 2$ corresponds to the application of the second narrow pulse. The combination $q \delta$ is kept fixed in the limit $\delta \rightarrow 0$, so that the magnetic field strength $q$ must go to infinity. For a linear magnetic field gradient, the macroscopic signal appears as the Fourier transform (13) of the Green function. A complete separation of the geometrical properties of the confining domain (represented by the Green function) and spatial

19 Balinov et al. (1994); Callaghan (1991); Callaghan et al. (1991); Coy and Callaghan (1994); King et al. (1994); Kuchel et al. (1996); Sen et al. (1995); Söderman and Jönsson (1995); Tanner and Stejskal (1968). See also references in subsection II.D. inhomogeneities of the magnetic field allowed to obtain a number of important theoretical results. This concept of " $q$-imaging" provided a direct experimental measurement of the Green function. ${ }^{20}$ In particular, one can expect an oscillatory behavior related to the geometrical structure of the confining medium (Callaghan, 1991). To illustrate this effect, one can again expand the Green function over the Laplace operator eigenbasis. In the case of uniform initial density $\rho(\mathbf{r})$, the macroscopic signal from (156) takes a simple matrix form:

$$
E=\left[e^{i q \delta \mathcal{B}} e^{-p \Lambda / 2} e^{-i q \delta \mathcal{B}}\right]_{0,0}
$$

The elements of the matrix $e^{i q \delta \mathcal{B}}$ were found explicitly for three basic domains (see Table I). For instance, the calculation for a slab geometry with reflecting boundary condition gives ${ }^{21}$

$$
\left[e^{i q \delta \mathcal{B}}\right]_{m, m^{\prime}}=\frac{\epsilon_{m} \epsilon_{m^{\prime}}}{2}\left[\psi_{\left|m-m^{\prime}\right|}(q \delta)+\psi_{m+m^{\prime}}(q \delta)\right],
$$

where

$$
\psi_{m}(q \delta)=\frac{(-1)^{m} q \delta}{(q \delta)^{2}-(\pi m)^{2}}\left[\sin q \delta+i\left((-1)^{m}-\cos q \delta\right)\right] .
$$

The substitution of this result into (157) leads to the classical form of the signal attenuation in a slab geometry within the narrow-pulse approximation:

$$
E=\frac{2(1-\cos q \delta)}{(q \delta)^{2}}+4(q \delta)^{2} \sum_{m=1}^{\infty} e^{-\pi^{2} m^{2} p / 2} \frac{1-(-1)^{m} \cos q \delta}{\left[(q \delta)^{2}-(\pi m)^{2}\right]^{2}}
$$

If the echo time $T$ is long enough $\left(p \gg 2 / \pi^{2}\right)$, the second term can be omitted, and the signal exhibits "diffractionlike" oscillations. Since the dimensionless parameter $q$ is defined as $\gamma g L T$, the behavior of the macroscopic signal as a function of the gradient intensity $g$ allows one to determine the distance $L$ between parallel plates of a slab geometry, i.e., to probe this geometry experimentally.

The applicability of the narrow-pulse approximation was thoroughly studied (Blees, 1994; Lori et al., 2003;

20 The " $q$-space" parameter $\gamma g \delta / 2 \pi$ introduced in Callaghan (1991) and frequently used in the literature on the narrow-pulse approximation should not be confused with our dimensionless parameter $q=\gamma g T L$.

21 The matrix $e^{i q \delta \mathcal{B}}$ naturally appears in the multiple propagator approach (see Appendix E). In the case of a cylinder and a sphere, its elements $\left[e^{i q \delta \mathcal{B}}\right]_{m, m^{\prime}}$ for any $m$ and $m^{\prime}$ were obtained by Codd and Callaghan (1999) for the Fourier boundary condition (10). Another representation for the Neumann boundary condition (11) was given by Sukstanskii and Yablonskiy (2002). In both cases, the expressions are cumbersome and have to be computed numerically. However, the calculation of the signal by Eq. (157) requires the knowledge of the elements $\left[e^{i q \delta \mathcal{B}}\right]_{0, m}$ alone, and their expressions are simpler (see Table I, L11). 


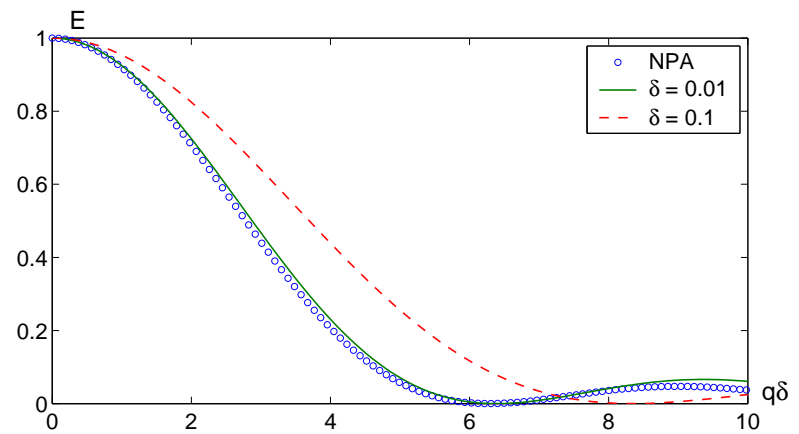

FIG. 6 Macroscopic signal as a function of $q \delta$ is found analytically within the narrow-pulse approximation (circles), and numerically by the MCF approach for $\delta=0.01$ (solid line) and $\delta=0.1$ (dashes line). The physical parameters $\left(D=2.3 \cdot 10^{-9} \mathrm{~m}^{2} / \mathrm{s}, L=1.6 \cdot 10^{-5} \mathrm{~m}, T=0.22 \mathrm{~s}\right)$ qualitatively correspond to that from (Callaghan et al., 1991). One sees that the approximate relation (160) is not applicable for $\delta=0.1$ since the condition $\delta p \ll 1$ fails (here $p \simeq 2$ ).

Mair et al., 2002; Malmborg et al., 2004; Price et al., 2003; Wang et al., 1995, etc.). Its practical application is limited by the assumption about a short duration $\delta T$ of gradient pulses with respect to the characteristic diffusion time $L^{2} / D$, i.e., $\delta p \ll 1$. If $p$ is too small, the diffraction effects are dumped by a number of terms in the sum. In fact, it can be shown that the relation (160) is reduced to $E \simeq 1-q^{2} p\left(\delta^{2} / 2\right)$ that can be written in a classical Gaussian form

$$
E \simeq \exp \left[-q^{2} p \delta^{2} / 2\right]
$$

In this case, there is no need to use the narrow-pulse approximation. In the opposite limit of large $p$, the diffraction effects would appear (Fig. 6), but the condition $\delta p \ll 1$ is more difficult to realize in experiment (since the gradient pulse duration $\delta$ is limited by instrumental constraints). In spite of this difficulty, the "diffusion diffraction" was experimentally observed for the first time in a water-saturated, orientationally disordered, loosely packed array of monodisperse polystyrene spheres by Callaghan et al. (1991).

The narrow-pulse approximation may also be valuable to give an intuitive feeling how the other more sophisticated techniques work. For instance, its use as a simplified temporal profile helps to better understand the properties of the $f$-weighted time average in the MCF approach. In the limit $\delta \rightarrow 0$, the $f$-weighted time average of a smooth function $F\left(t_{1}, \ldots, t_{n}\right)$ is reduced to

$$
<F\left(t_{1}, \ldots, t_{n}\right)>_{n} \simeq \frac{\delta^{n}}{n !} \sum_{k=0}^{n}(-1)^{k} C_{n}^{k} F(0,0, \ldots, \underbrace{\frac{1}{2}, \frac{1}{2}}_{k \text { times }}),
$$

where $C_{n}^{k}$ are the binomial coefficients. For example,

$$
\left.<F\left(t_{1}, t_{2}\right)\right\rangle_{2} \simeq \frac{\delta^{2}}{2}\left(F(0,0)-2 F\left(0, \frac{1}{2}\right)+F\left(\frac{1}{2}, \frac{1}{2}\right)\right) .
$$

This general result can now be used to write the moments as

$$
\begin{aligned}
\mathbb{E}\left\{\frac{\phi^{n}}{n !}\right\} \simeq \frac{\delta^{n}}{n !}( & \left(1+(-1)^{n}\right)\left[\mathcal{B}^{n}\right]_{0,0} \\
& \left.+\sum_{k=1}^{n-1}(-1)^{k} C_{n}^{k}\left[\mathcal{B}^{k} e^{-p \Lambda / 2} \mathcal{B}^{n-k}\right]_{0,0}\right)
\end{aligned}
$$

A further analysis is significantly simplified by using this relation. For instance, one can easily check that all odd moments vanish since $\left[\mathcal{B}^{k} e^{-p \Lambda / 2} \mathcal{B}^{n-k}\right]_{0,0}=$ $\left[\mathcal{B}^{n-k} e^{-p \Lambda / 2} \mathcal{B}^{k}\right]_{0,0}$. Note that the relation (164) could be derived directly from Eq. (156) by using the spectral decomposition (25) of the Green function within the narrow-pulse approximation.

Another representation for the even moments can be derived by expanding the exponential function in (156):

$$
\begin{aligned}
\mathbb{E}\left\{\frac{\phi^{2 n}}{(2 n) !}\right\}=\frac{\delta^{2 n}}{(2 n) !} \int_{\Omega} d \mathbf{r}_{0} & \rho\left(\mathbf{r}_{0}\right) \int_{\Omega} d \mathbf{r} G_{T / 2}\left(\mathbf{r}_{0}, \mathbf{r}\right) \times \\
& \times\left[B\left(\mathbf{r}_{0}\right)-B(\mathbf{r})\right]^{2 n}
\end{aligned}
$$

The spectral decomposition of the Green function allows one to study the dependence of these moments on the parameter $p$. In particular, one can rigorously obtain their leading terms (see Appendix F for a sketch of this derivation):

$$
\mathbb{E}\left\{\frac{\phi^{2 n}}{(2 n) !}\right\} \simeq \frac{p^{n}}{n !}\left(\delta^{2} / 2\right)^{n}\left(\frac{L^{2 n}}{V} \int_{\Omega} d \mathbf{r}|\nabla B(\mathbf{r})|^{2 n}\right) .
$$

This is a particular form of the general relation (76) in the case of the narrow-pulse profile, for which the $f$-weighted time average $\left\langle\left(t_{1}-t_{2}\right)\right\rangle_{2}$ is $\delta^{2} / 2$. While the general result was demonstrated for $n=1,2$ and conjectured for higher $n$, the relation (166) is proven for any $n$. This example may illustrate the use of the narrow-pulse approximation as an investigation tool.

\section{F. Localization regime}

In previous sections, we have considered the behavior of the moments $\mathbb{E}\left\{\phi^{2 n}\right\}$ in two asymptotic limits, when the dimensionless diffusion coefficient $p$ goes to zero or infinity. In both cases, the leading terms could be satisfactory only for very small or very high values of $p$. The use of correction terms significantly improved the quality of the results, but was still insufficient to describe the behavior for intermediate values of $p$. Moreover, our analysis was essentially focused on the second and fourth moments. If the dimensionless magnetic field strength $q$ is small, these moments provide the most significant contribution to the signal and contain exhaustive information about its attenuation. However, when $q$ 
increases, a larger number of moments is needed to accurately compute the signal $E$. One may thus expect to observe a new kind of behavior for sufficiently intense magnetic fields. This is actually the case. If the dephasing length $(D / \gamma g)^{1 / 3}$ is much smaller than the diffusion length $\sqrt{D T}$ and the characteristic dimension $L$ of the domain, a new localization regime appears (de Swiet and Sen, 1994; Stoller et al., 1991). In this case, the spins of the "bulk" diffuse over several dephasing lengths so that their net contribution to the total magnetization vanishes. The macroscopic signal is thus formed by the spins close to the interface whose dephasing is less pronounced. In our notation, this situation corresponds to $q \gg p$ and $q^{2} p \gg 1$.

Stoller et al. (1991) gave the first theoretical study of this regime for one-dimensional diffusion. Using an exact resolution of the one-dimensional Bloch-Torrey equation (9) and analyzing of the underlying spectral problem, they showed a non-Gaussian behavior of the signal $E$ :

$$
E \simeq C(p / q)^{1 / 3} \exp \left[-\frac{E_{1}}{2}\left(p q^{2}\right)^{1 / 3}\right],
$$

where $E_{1} \simeq 1.0188$ is the first root of the derivative of the Airy function. The numerical prefactor $C$ was found to be 5.884 for a slab geometry. The dependence (167) and its significance for NMR applications were discussed by different authors (de Swiet and Sen, 1994; Hürlimann et al., 1995; Sen et al., 1999). In particular, the coefficient $E_{1} / 2$ was argued to be independent of the confining geometry.

Four years later, the above theoretical prediction was observed by spin-echo technique. An elegant experiment of Hürlimann et al. (1995) definitely destroyed the myth of "almighty" Gaussian phase approximation and dispelled last doubts about the localization regime. Hürlimann et al. studied restricted diffusion of water molecules $\left(D \simeq 2.3 \cdot 10^{-9} \mathrm{~m}^{2} / \mathrm{s}\right.$ ) between two parallel plates at distance $L=0.16 \mathrm{~mm}$. The signal attenuation was measured as a function of the gradient intensity $g$. Even for long-time gradient pulses $(T=120 \mathrm{~ms})$, the dimensionless diffusion coefficient $p$ was small $(p \simeq 0.01)$ so that the slow diffusion regime, with a Gaussian $g^{2}$ dependence, could be expected. This was actually observed for the gradient applied in a longitudinal (unrestricted) direction. Figure 7 shows a good agreement between experimental points (squares) and theoretical prediction (dash-dotted line) by the classical Hahn's relation (3).

The situation became completely different when the gradient had been turned on the direction perpendicular to the plates (circles in Fig. 7). A drastic deviation from the Gaussian $g^{2}$-dependence of the $\ln E$ can be clearly seen at gradient intensity higher than $15 \mathrm{mT} / \mathrm{m}\left(q^{2} \geq\right.$ $\left.0.6 \cdot 10^{4}\right)$. This behavior cannot be attributed neither to the slow diffusion limit, nor to the motional narrowing regime. Neither an apparent diffusion coefficient, nor any related concept has meaning in this localization regime.
Figure 7 shows that the experimental data are in a qualitative agreement with the predictions by Stoller et al. (diamonds). A small deviation from the theoretical relation (167) is mainly caused by surface relaxation. To illustrate this point, we have numerically calculated the macroscopic signal by MCF approach. The dashed line, corresponding to the Neumann boundary condition (11), confirms a good precision of the Stoller's theory in the case when the surface relaxation can be neglected $(K=0$ or $h=0)$. To fit better the experimental points, we calculated the signal for different values of the dimensionless surface relaxation $h$. The solid line corresponds to the Fourier boundary condition (10) with $h=2$ (or $K \simeq 28.8 \mu \mathrm{m} / \mathrm{s}){ }^{22}$

The most exciting feature of the observation by Hürlimann et al. is that the localization regime is observed under ordinary experimental conditions. In view of the above results, a number of theoretical questions arises. A formal expansion of the exponential function in (167) leads to a series of fractional powers of $p$ and $q$. This is probably not surprising with respect to $p$, since we have already seen fractional powers of $p$ in the correction term to the slow diffusion regime. In contrast, the fractional powers of $q$ would be in contradiction to the fact that the signal $E$ must be an analytical function of $q$. Of course, the relation (167) has to be considered as an asymptotic behavior for $p q^{2} \gg 1$ so that the above expansion is not formally allowed. Nevertheless, the passage from the general expansion (46) to this asymptotic form is intriguing. In spite of a considerable theoretical work, this question remains still open. The MCF approach may help to shed a new light on this interesting problem. Since this description is not restricted to a particular choice of parameters $p$ and $q$, it can reproduce, at least numerically, any feature of the signal attenuation, including the localization regime. Its theoretical analysis would require a systematic technique for calculating the moments $\mathbb{E}\left\{\phi^{n}\right\}$ of high orders.

At first sight, the problematic of the localization regime may look like a mathematical puzzle of limited practical interest. We believe, however, that a better understanding of the nature of this specific non-Gaussian behavior will enrich our knowledge about diffusive NMR phenomena in general. Moreover, some recent researches showed a potential importance of the localization regime for restricted diffusion in lungs (Grebenkov and Guillot, 2006).

22 This value of the surface relaxation $K$ is given for illustrative purpose only. A more profound analysis of the localization regime in presence of surface relaxation is certainly required and will be helpful to accurately determine this characteristic. Some other possible sources of deviation between the theory and the experiment were mentioned by Hürlimann et al. (1995). 


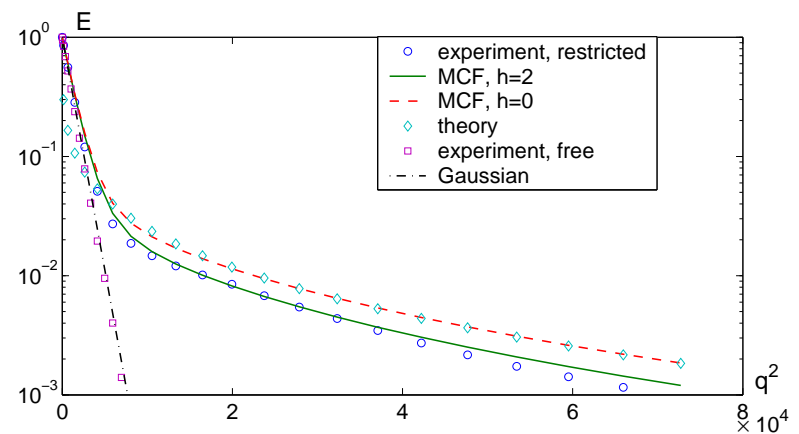

FIG. 7 Illustration of a drastic deviation from a Gaussian behavior at high gradient intensity. At logarithmic scale, experimental data for free diffusion (when the gradient applied along unrestricted direction) fall onto the straight line $-q^{2} p / 12$ as expected. In contrast, experimental data for restricted diffusion follow the theoretical relation (167). A small deviation from this behavior is mainly caused by surface relaxation as shown by numerical analysis. The shown experimental data from (Hürlimann et al., 1995), have been communicated to the author by Dr. M. D. Hürlimann.

\section{G. Unrestricted diffusion}

The unrestricted diffusion ("ordinary" Brownian motion in the whole space) was always considered as the most simple situation for theoretical analysis since the Green function $G_{t}\left(\mathbf{r}, \mathbf{r}^{\prime}\right)$ takes an explicit Gaussian form:

$$
G_{t}\left(\mathbf{r}, \mathbf{r}^{\prime}\right)=(4 \pi D t)^{-d / 2} \exp \left[-\frac{\left(\mathbf{r}-\mathbf{r}^{\prime}\right)^{2}}{4 D t}\right]
$$

$d$ being the dimension of the space. Unfortunately, the unrestricted diffusion brings a number of specific unphysical artifacts like infinite volume of the bulk or infinite magnetic field in the case of a linear gradient. Moreover, the use of a usually appropriate uniform spin density would require an infinite amount of spins producing infinite signal at time $t=0$. Indeed, the exact solution of the Bloch-Torrey equation (9) in the one-dimensional space $\mathbb{R}$ under steady magnetic field $B(x)=g x$ of linear gradient $g$ (without rephasing condition) gives the magnetization density $\mathfrak{m}(x, t)$ as

$$
\mathfrak{m}(x, t)=\exp [i \gamma g x t] \exp \left[-D \gamma^{2} g^{2} t^{3} / 3\right]
$$

(Borodin and Salminen, 1996; Torrey, 1956). The second factor can be easily recognized as Hahn's attenuation (Hahn, 1950). However, the integration of the above relation over the bulk $\mathbb{R}$ with uniform initial density gives a delta-function $\delta(\gamma g t)$ which has a simple physical meaning. At time $t=0$, an infinite amount of spins are in phase producing an infinite macroscopic signal. At late times $t>0$, the dephasing of an infinity of spins totally cancels the signal. In the literature, some tricks have been suggested to overcome this divergence. In our opinion, the only rigorous way to avoid such difficulties is to work directly with confining domains.
In this light, the passage from restricted to unrestricted diffusion is delicate. In particular, for typical magnetic fields $B(\mathbf{r})$, the correlation functions $\mathbb{E}\left\{B\left(X_{t_{1}}\right) \ldots B\left(X_{t_{n}}\right)\right\}$ given by Eq. (24) diverge after integration with uniform initial density $\rho\left(\mathbf{r}_{0}\right)$. On the other hand, the Stejskal-Tanner formula (90) for the macroscopic signal is independent of the characteristic dimension of the domain in the case of linear gradients, and can thus be applied for unrestricted diffusion. A similar argument fails in other cases, e.g., for a parabolic magnetic field.

\section{CONCLUSION}

The fascinating properties of the Brownian motion have attracted scientists for more than one and a half centuries from its discovery in 1827. Among a variety of related scientific domains, nuclear magnetic resonance provides an efficient experimental tool to survey the diffusive motion by a direct measurement of different functionals of this stochastic process. A voluntary choice of the temporal and spatial profiles of the applied magnetic field makes possible, in principle, a complete experimental analysis of Brownian trajectories. The presence of a confining boundary makes this problem still more intriguing. On the other hand, the specific properties of the reflected Brownian motion are at the origin of the diversity and complexity of diffusive NMR phenomena observed in experiments. In spite of intensive theoretical investigation during the last five decades, a lot of important questions remain open.

The main aim of this review was to show in an original unified way the progressive development in this field from the Hahn's discovery of spin echoes till nowadays. For this purpose, the multiple correlation function approach was first established and then used to retrieve, extend and critically discuss a number of classical results. This approach makes possible a theoretical analysis of restricted diffusion in confining media under arbitrary magnetic field. Its spatial inhomogeneities are naturally included via matrix elements $\mathcal{B}_{m, m^{\prime}}$ in the Laplace operator eigenbasis. In turn, the dependence on time was taken into account as the $f$-weighted time average of the multiple correction functions. A thorough analysis of the second and higher order moments of the random phase accumulated by a diffusing spin in magnetic field allowed us to retrieve some classical results in a more general form. For instance, the slow diffusion and motional narrowing regimes were extended to arbitrary temporal and spatial profiles of the magnetic field. A further analysis of high order moments will allow to specify the validity ranges of the Gaussian phase approximation. The study of corrections to the leading terms also gave new results. In the slow diffusion regime, the results by Mitra et al. have been generalized for arbitrary temporal and spatial profiles. The obtained relations were reinterpreted in order to precise the meaning of the surface-to-volume 
ratio which appeared as a prefactor in the correction term. In the motional narrowing regime, the classical corrections, first found by Robertson (1966) and Neuman (1974), have been extended and critically discussed. In particular, we have shown that the form of these corrections strongly depends on the temporal profile of the magnetic field. The slow diffusion correction term and a simple interpolation formula have been compared to experimental measurements of the apparent diffusion coefficient in a cylindrical cell. Although we could proceed far enough, a systematic computational technique to operate with the temporal and spatial averages for high order moments is still required.

Two simplifying assumptions were used to advance the analysis. The computation of the $f$-weighted time averages was significantly easier due to the rephasing condition which, in turn, was required for echo formation. The derivation of the results for the fourth moment and the cancellation of odd moments were mainly based on this condition. The second assumption about the Neumann boundary condition concerned the spatial averages. For instance, an analytical computation of the coefficients $\zeta_{k}$ called for the fact that the ground eigenstate $u_{0}$ was constant (and the corresponding eigenvalue was zero). In principle, one could try to reproduce the analytical results of this paper in a more general context, including relaxing interfaces or non-rephased temporal profiles. However, this effort seems to be rather of an academic interest since the numerical analysis of the problem can be easily performed in a general case.

The lack of a complete theory of restricted diffusion in confining media under arbitrary magnetic field was probably one of the main obstacles in developing experimental NMR techniques with specifically designed temporal and spatial profiles. The use of nonlinear fields beyond a simple linear gradient may help to enhance the particular geometrical features of a sample. For instance, a spatial profile with two local minima at fixed locations or with a local "valley" would enable one to study the diffusive motion of spins between these locations (since the contribution of other spins would be much more attenuated by higher magnetic field). A periodic distribution of $B(\mathbf{r})$ might be also of practical interest to enhance diffusion diffraction. At the same time, the susceptibilityinduced or random magnetic fields can now be treated in an efficient numerical way. This would be a promising extension of some interesting techniques, ${ }^{23}$ developed originally for the whole space, to a more realistic case of confining media. On the other hand, an opportunity of using various temporal profiles of the magnetic field is not explored yet. While the induction of a nonlinear magnetic field presents an experimental challenge for itself, the design of specific temporal profiles is somewhat

23 (Kiselev and Posse, 1998; Kiselev and Novikov, 2002; Mitra and Le Doussal, 1991; Sukstanskii and Yablonskiy, 2003, 2004). readily accessible. For instance, the temporal profile optimization might be useful to increase the contribution of the correction term in the slow diffusion regime.

The MCF approach creates a mathematical basis for a new promising branch of computational NMR analysis. Combined with numerical tools in electrodynamics, it makes possible a complete study of the diffusive phenomena in coils which can be specifically designed to generate nonlinear diffusion-sensitizing magnetic fields. Indeed, one can first calculate the spatial profile of the magnetic field in a chosen geometry, and then use this profile to predict the consequent signal attenuation in a NMR experiment. This analysis will help to design the gradient coils whose selective properties will be adapted to enhance a particular property of the restricted motion.

The scientific interest of diffusive NMR phenomena is actually far beyond their role for the experimental study of porous materials or biological tissues. As we have shown in this review, a close relation to the probability theory should make them attractive for mathematicians investigating the reflected Brownian motion. From this methodological point of view, a rigorous reformulation of numerous physical results dispersed in the literature seems to be of great importance to bring new ideas in this long-standing field. A number of open mathematical problems were outlined in previous sections. Among them, we should stress a better understanding of the transition between different diffusion limits, especially the passage to the localization regime. At last, the inverse problem of determining the confining geometry from the knowledge of the signal attenuation is unsolved and of primary practical importance, for instance, to detect the mineral oil in rocky structures or pathological diseases in human organs.

\section{Acknowledgments}

The author is grateful to Dr. G. Guillot, Dr. X. Maître and Dr. P. Tankov for valuable discussions and careful reading of the manuscript. A special acknowledgment is to Dr. M. Hayden and Dr. M. D. Hürlimann for sending to the author a set of experimental data to illustrate different NMR regimes. This work has been supported by EU Network MRTN-CT-2003-504712, the ANR project "MIPOMODIM nNT05-1_42030" and Ecole Polytechnique (Palaiseau, France).

\section{APPENDIX A: Fourth moment in the slow diffusion regime}

In the slow diffusion regime, the leading term of the fourth moment is expected to be of order of $p^{2}$. To show it, the exponential functions in (51) can be formally expended up to second orders in $p$. The $f$-weighted average of a constant (order $p^{0}$ ) vanishes due to the rephasing condition. We first show that there is no contribution of the first order, and then calculate the coefficient in front 
of $p^{2}$.

\section{First order contribution}

There are three terms of order $p$ :

$$
\begin{aligned}
& {\left[\mathcal{B} \Lambda \mathcal{B}^{3}\right]_{0,0}<\left(t_{2}-t_{1}\right)>_{4}+\left[\mathcal{B}^{2} \Lambda \mathcal{B}^{2}\right]_{0,0} \times} \\
& \times<\left(t_{3}-t_{2}\right)>_{4}+\left[\mathcal{B}^{3} \Lambda \mathcal{B}\right]_{0,0}<\left(t_{4}-t_{3}\right)>_{4}
\end{aligned}
$$

The spatial averages are found by a field theory technique briefly outlined in subsection IV.A:

$$
\begin{aligned}
{\left[\mathcal{B}^{3} \Lambda \mathcal{B}\right]_{0,0} } & =\left[\mathcal{B} \Lambda \mathcal{B}^{3}\right]_{0,0}=\frac{3}{4}\left[\mathcal{B}^{2} \Lambda \mathcal{B}^{2}\right]_{0,0}= \\
& =\frac{3 L^{2}}{V} \int_{\Omega} d \mathbf{r} B^{2}(\mathbf{r})|\nabla B(\mathbf{r})|^{2} .
\end{aligned}
$$

The $f$-weighted time averages are

$$
\begin{aligned}
& <\left(t_{2}-t_{1}\right)>_{4}=<\left(t_{4}-t_{3}\right)>_{4}= \\
& =-\frac{2}{3}<\left(t_{3}-t_{2}\right)>_{4}=-\frac{1}{6} F_{4},
\end{aligned}
$$

where

$$
F_{k}=\int_{0}^{1} d t[\hat{f}(t)]^{k}
$$

and the primitive

$$
\hat{f}(t)=\int_{0}^{t} d t^{\prime} f\left(t^{\prime}\right)
$$

satisfies $\hat{f}(0)=\hat{f}(1)=0$ due to the rephasing condition (36). Consequently, the sum of the three terms in (A1) is strictly zero. We have thus shown that there is no contribution of the first order in $p$.

\section{Second order contribution}

The second order contribution to a formal series expansion is given by the first diagonal element of the matrix

$$
\begin{aligned}
& \frac{1}{2}\left(t_{2}-t_{1}\right)^{2} \mathcal{B} \Lambda^{2} \mathcal{B}^{3}+\frac{1}{2}\left(t_{3}-t_{2}\right)^{2} \mathcal{B}^{2} \Lambda^{2} \mathcal{B}^{2}+ \\
& \frac{1}{2}\left(t_{4}-t_{3}\right)^{2} \mathcal{B}^{3} \Lambda^{2} \mathcal{B}+\left(t_{2}-t_{1}\right)\left(t_{3}-t_{2}\right) \mathcal{B} \Lambda \mathcal{B} \Lambda \mathcal{B}^{2}+ \\
& \left(t_{2}-t_{1}\right)\left(t_{4}-t_{3}\right) \mathcal{B} \Lambda \mathcal{B}^{2} \Lambda \mathcal{B}+\left(t_{3}-t_{2}\right)\left(t_{4}-t_{3}\right) \mathcal{B}^{2} \Lambda \mathcal{B} \Lambda \mathcal{B} .
\end{aligned}
$$

where the $f$-weighted time average has to be taken. The computation of this coefficient in front of $p^{2}$ is complicated by the fact that the six individual terms in this expression are divergent (while their combination should converge). One thus need to use a renormalization procedure to regroup these terms in a convergent way.

The computation of the $f$-weighted time averages is simple:

$$
\begin{aligned}
<\left(t_{2}-t_{1}\right)^{2}>_{4} & =-\frac{1}{6} F_{1} F_{3}, \\
<\left(t_{3}-t_{2}\right)^{2}>_{4} & =\frac{1}{4} F_{2}^{2}, \\
<\left(t_{4}-t_{3}\right)^{2}>_{4} & =-\frac{1}{6} F_{1} F_{3}, \\
<\left(t_{2}-t_{1}\right)\left(t_{3}-t_{2}\right)>_{4} & =\frac{1}{4}\left(F_{1} F_{3}-F_{2}^{2}\right), \\
<\left(t_{2}-t_{1}\right)\left(t_{4}-t_{3}\right)>_{4} & =-\frac{1}{2}\left(F_{1} F_{3}-F_{2}^{2}\right), \\
<\left(t_{3}-t_{2}\right)\left(t_{4}-t_{3}\right)>_{4} & =\frac{1}{4}\left(F_{1} F_{3}-F_{2}^{2}\right) .
\end{aligned}
$$

A field theory technique used in subsection IV.A to derive the relations (70), (72) can be applied here to calculate the spatial averages. For example, the first diagonal element of the matrix $\mathcal{B} \Lambda^{2} \mathcal{B}^{3}$ can be written as

$$
\sum_{m} \int_{\Omega} d \mathbf{r}_{1} \int_{\Omega} d \mathbf{r}_{2} B\left(\mathbf{r}_{1}\right) u_{m}^{*}\left(\mathbf{r}_{1}\right) \lambda_{m}^{2} u_{m}\left(\mathbf{r}_{2}\right) B^{3}\left(\mathbf{r}_{2}\right),
$$

where the sum is taken from $m=1$ to a large but finite cut-off. As previously, one replaces $\lambda_{m} u_{m}^{*}\left(\mathbf{r}_{1}\right)$ by $-L^{2} \Delta u_{m}^{*}\left(\mathbf{r}_{1}\right)$ and $\lambda_{m} u_{m}\left(\mathbf{r}_{2}\right)$ by $-L^{2} \Delta u_{m}\left(\mathbf{r}_{2}\right)$, and then uses the second Green formula for both integrals. The result can be conveniently represented as

$$
\left[\mathcal{B} \Lambda^{2} \mathcal{B}^{3}\right]_{0,0}=3 \sum_{m}\left(2 X_{m}^{(2)}+Y_{m}^{(2)}-Z_{m}^{(2)}\right)^{*}\left(Y_{m}^{(0)}-Z_{m}^{(0)}\right)
$$

where the following notations are introduced to lighten the expressions:

$$
\begin{aligned}
X_{m}^{(k)} & =L^{2} \int_{\Omega} d \mathbf{r} u_{m}(\mathbf{r}) B^{k-1}(\mathbf{r})|\nabla B(\mathbf{r})|^{2}, \\
Y_{m}^{(k)} & =L^{2} \int_{\Omega} d \mathbf{r} u_{m}(\mathbf{r}) B^{k}(\mathbf{r}) \Delta B(\mathbf{r}), \\
Z_{m}^{(k)} & =L^{2} \int_{\partial \Omega} d \mathbf{r} u_{m}(\mathbf{r}) B^{k}(\mathbf{r}) \frac{\partial}{\partial n} B(\mathbf{r}) .
\end{aligned}
$$

In a similar way, one finds five other spatial averages

$$
\begin{aligned}
& {\left[\mathcal{B}^{2} \Lambda^{2} \mathcal{B}^{2}\right]_{0,0}=4 \sum_{m}\left|X_{m}^{(1)}+Y_{m}^{(1)}-Z_{m}^{(1)}\right|^{2},} \\
& {\left[\mathcal{B}^{3} \Lambda^{2} \mathcal{B}\right]_{0,0}=3 \sum_{m}\left(2 X_{m}^{(2)}+Y_{m}^{(2)}-Z_{m}^{(2)}\right)^{*}\left(Y_{m}^{(0)}-Z_{m}^{(0)}\right),} \\
& {\left[\mathcal{B}^{2} \Lambda \mathcal{B} \Lambda \mathcal{B}\right]_{0,0}=2 \sum_{m}\left(X_{m}^{(2)}+Y_{m}^{(2)}-Z_{m}^{(2)}\right)^{*}\left(Y_{m}^{(0)}-Z_{m}^{(0)}\right),} \\
& {\left[\mathcal{B} \Lambda \mathcal{B}^{2} \Lambda \mathcal{B}\right]_{0,0}=\sum_{m}\left(Y_{m}^{(2)}-Z_{m}^{(2)}\right)^{*}\left(Y_{m}^{(0)}-Z_{m}^{(0)}\right),} \\
& {\left[\mathcal{B} \Lambda \mathcal{B} \Lambda \mathcal{B}^{2}\right]_{0,0}=2 \sum_{m}\left(X_{m}^{(2)}+Y_{m}^{(2)}-Z_{m}^{(2)}\right)^{*}\left(Y_{m}^{(0)}-Z_{m}^{(0)}\right) .}
\end{aligned}
$$


The substitution of the temporal and spatial averages into (A6) and further algebraic simplification gives the leading term of the fourth moment as

$$
\begin{aligned}
\mathbb{E}\left\{\frac{\phi^{4}}{4 !}\right\}=\frac{1}{2} p^{2} F_{2}^{2} \sum_{m}\left[\left|X_{m}^{(1)}+Y_{m}^{(1)}-Z_{m}^{(1)}\right|^{2}-\right. \\
\left.\left(2 X_{m}^{(2)}+Y_{m}^{(2)}-Z_{m}^{(2)}\right)^{*}\left(Y_{m}^{(0)}-Z_{m}^{(0)}\right)\right]
\end{aligned}
$$

This expression does not contain divergent terms, so that the sum can be extended up to infinity. One then substitutes the notations (A10) and sums over $m$ to get $\delta\left(\mathbf{r}_{1}-\mathbf{r}_{2}\right)$. After integration, a number of terms in the above expression vanish. One finally gets the leading terms of the fourth moment:

$\mathbb{E}\left\{\frac{\phi^{4}}{4 !}\right\}=\frac{1}{2} p^{2}\left(<\left(t_{1}-t_{2}\right)>_{2}\right)^{2}\left(\frac{L^{4}}{V} \int_{\Omega} d \mathbf{r}|\nabla B(\mathbf{r})|^{4}\right)$,

where the $f$-weighted time average $\left\langle\left(t_{1}-t_{2}\right)\right\rangle_{2}$ is substituted instead of $F_{2}$. A similar analysis could be used to compute higher moments, but a more systematic technique would be certainly helpful.

\section{APPENDIX B: Laplace transform technique}

In subsection IV.C, the $p^{3 / 2}$ correction terms to the second moment has been obtained in the slow diffusion regime $(p \ll 1)$. Here, we briefly show how the Laplace transform technique can be used to study the second order moment in a more precise way. ${ }^{24}$ In particular, we are looking for the spatial average $\left[\mathcal{B} e^{-p\left(t_{2}-t_{1}\right) \Lambda} \mathcal{B}\right]_{0,0}$ and its dependence on the variable $\tilde{p}=p\left(t_{2}-t_{1}\right)$. For three basic domains and two choices of the magnetic fields spatial profiles, one has

$$
\left[\mathcal{B} e^{-p\left(t_{2}-t_{1}\right) \Lambda} \mathcal{B}\right]_{0,0}=\left(\mathcal{B}_{0,0}\right)^{2}+H(\tilde{p}),
$$

where

$$
H(\tilde{p})=c_{h} \sum_{m=1}^{\infty} \frac{e^{-\tilde{p} \alpha_{m}^{2}}}{\alpha_{m}^{2}\left(\alpha_{m}^{2}-\alpha\right)}
$$

and the numerical prefactor $c_{h}$, constant $\alpha$ and values $\alpha_{m}$ are determined by the matrices $\mathcal{B}$ and $\Lambda$ (see Table IV).

Let us denote by $\mathcal{L}[H](s)$ the Laplace transform of the function $H(\tilde{p})$

$$
\mathcal{L}[H](s)=\int_{0}^{\infty} d \tilde{p} e^{-s \tilde{p}} H(\tilde{p}) .
$$

24 This technique was applied by Mitra et al. (1993) to calculate the time-dependent diffusion coefficient for a sphere.
It is easy to check that the Laplace transform of the derivative of $H(\tilde{p})$ with respect to $\tilde{p}$ is equal to

$$
\mathcal{L}\left[H^{\prime}\right](s)=\frac{-c_{h}}{s+\alpha} \sum_{m=1}^{\infty}\left(\frac{1}{\alpha_{m}^{2}-\alpha}-\frac{1}{\alpha_{m}^{2}+s}\right) .
$$

In the first sum, one can recognize $[\mathcal{B} \Lambda \mathcal{B}]_{0,0}$ that was denoted as $\zeta_{1}$. If one introduces a new function

$$
H_{0}(\tilde{p})=\sum_{m=1}^{\infty} e^{-\tilde{p} \alpha_{m}^{2}}
$$

the second sum can be written as $\mathcal{L}\left[H_{0}\right](s)$. On the other hand, the integration by parts in the definition of the Laplace transform gives $\mathcal{L}\left[H^{\prime}\right](s)=-H(0)+s \mathcal{L}[H](s)$, where $H(0)$ was denoted as $\zeta_{0}$. One thus obtains

$$
\mathcal{L}[H](s)=\frac{\zeta_{0}}{s}-\frac{\zeta_{1}}{s(s+\alpha)}+\frac{c_{h} \mathcal{L}\left[H_{0}\right](s)}{s(s+\alpha)} .
$$

Taking the inverse Laplace transform, one gets $^{25}$

$$
\begin{array}{r}
{\left[\mathcal{B} e^{-p\left(t_{2}-t_{1}\right) \Lambda} \mathcal{B}\right]_{0,0}=\tilde{\zeta}_{0}-\frac{\zeta_{1}}{\alpha}\left(1-e^{-p\left(t_{2}-t_{1}\right) \alpha}\right)} \\
+c_{h} \mathcal{L}^{-1}\left\{\frac{\mathcal{L}\left[H_{0}\right](s)}{s(s+\alpha)}\right\}\left(p\left(t_{2}-t_{1}\right)\right) .
\end{array}
$$

Consequently, the spatial average on the left hand side is related to the inverse Laplace transform of the function $\mathcal{L}\left[H_{0}\right](s) /(s(s+\alpha))$. Curiously, $\mathcal{L}\left[H_{0}\right](s)$ can be found explicitly for three basic domains ${ }^{26}$ (see Table IV). In this light, Eq. (B7) is a closed and relatively simple relation, which does not involve an infinite set of the roots $\alpha_{m}$. However, numerical computation of rapidly convergent series in (B2) is in general much easier than finding the inverse Laplace transform in (B7).

In the slow diffusion limit $p \rightarrow 0$ (or $s \rightarrow \infty$ ), one can use the relation (B7) to rigorously obtain the correction term. For three basic domains and two choices of the magnetic field spatial profile, one finds

$$
\mathcal{L}\left[H_{0}\right](s) \simeq c_{\ell} s^{-1 / 2} \quad(s \rightarrow \infty) .
$$

The numerical prefactor $c_{\ell}$ is equal to $1 / 2$ for all cases, except for a slab geometry with a linear gradient, for

25 The case $\alpha=0$ should be considered as a limit $\alpha \rightarrow 0$.

26 In general, the calculation of the sum

$$
\sum_{m=1}^{\infty} \frac{2 x}{x^{2}-\alpha_{m}^{2}}=\sum_{m=1}^{\infty}\left(\frac{1}{x-\alpha_{m}}+\frac{1}{x+\alpha_{m}}\right)
$$

is based on the fact that $\pm \alpha_{m}$ are all the roots of some explicit function (e.g., $J_{1}^{\prime}(x)$ in the case of a cylinder with linear magnetic field gradient, see (Watson, 1962) for details). The substitution $x=i \sqrt{s}$ leads then to the results shown in Table IV. If $x=0$ is also the root, a supplementary term appears. 
which $c_{\ell}=1 / 4$. One gets

$$
\begin{aligned}
& {\left[\mathcal{B} e^{-p\left(t_{2}-t_{1}\right) \Lambda} \mathcal{B}\right]_{0,0} \simeq \tilde{\zeta}_{0}-\zeta_{1} p\left(t_{2}-t_{1}\right)+} \\
& +\frac{4 c_{h} c_{\ell}}{3 \sqrt{\pi}} p^{3 / 2}\left(t_{2}-t_{1}\right)^{3 / 2}+O\left(p^{2}\right),
\end{aligned}
$$

where the identity $\mathcal{L}^{-1}\left[s^{-\beta}\right](\tilde{p})=\tilde{p}^{\beta-1} / \Gamma(\beta)$ was used. One thus retrieves the results of subsection IV.C with

$$
\mu_{2}=\frac{4 c_{h} c_{\ell}}{3 \sqrt{\pi}}
$$

Correction terms of order $p^{2}$ and higher can be derived in a similar way (see Table IV).

It is worth to note that Eq. (B8) is equivalent to the asymptotic behavior of $H(\tilde{p})$ at $\tilde{p} \rightarrow 0$ :

$$
H_{0}(\tilde{p}) \simeq \frac{c_{\ell}}{(\pi \tilde{p})^{1 / 2}} .
$$

At this point, we outline an interesting similarity between this function and the averaged return-to-the-origin probability considered by Mitra et al. (1995b) and by Schwartz et al. (1997):

$$
P(\tilde{p})=\int_{\Omega} d \mathbf{r} G_{t_{2}-t_{1}}(\mathbf{r}, \mathbf{r})=\sum_{m=0}^{\infty} e^{-\tilde{p} \lambda_{m}} .
$$

The only difference is that the last sum contains all eigenvalues $\lambda_{m}$, while the function $H_{0}(\tilde{p})$ is defined for specific eigenmodes, for which $\mathcal{B}_{0, m} \neq 0$. In the limit $\tilde{p} \rightarrow 0$, the Green function in (B12) can be roughly approximated by the free diffusion Green function (168), whence $P(\tilde{p}) \sim(4 \pi \tilde{p})^{-d / 2}$. Since $\mathcal{L}\left[H_{0}\right](s)$ contains a smaller number of terms, its convergence at $s \rightarrow \infty$ is better, and the behavior of $H_{0}(\tilde{p})$ at $\tilde{p} \rightarrow 0$ is less sharp than for $P(\tilde{p})$. Note that the properties of the function $P(\tilde{p})$ was investigated by Kac (1966) in attempt to answer the famous question: "Can one hear the shape of a drum?". Although interesting, a further discussion on this topic is out of the scope of this paper.

\section{APPENDIX C: Fourth moment in the motional narrowing regime}

As an illustrative example, we are going to calculate the leading term of the fourth moment $\mathbb{E}\left\{\phi^{4}\right\}$ in the motional narrowing regime. Although the calculation is elementary as well as for the second moment, the variety of specific cases makes it cumbersome for higher moments.

Let us consider the $f$-weighted time average for the fourth moment:

$$
<e^{-p \lambda_{m_{1}}\left(t_{2}-t_{1}\right)} e^{-p \lambda_{m_{2}}\left(t_{3}-t_{2}\right)} e^{-p \lambda_{m_{3}}\left(t_{4}-t_{3}\right)}>_{4} .
$$

As for the second moment, one might simply replace each exponential function by its leading asymptotic term $\left(p \lambda_{m_{i}}\right)^{-1} \delta\left(t_{i+1}-t_{i}\right)$ as $p \rightarrow \infty$, so that the fourth moment would behave as $p^{-3}$. However, a more accurate analysis requires to account for special cases when certain $\lambda_{m_{i}}$ are zero. Let us briefly consider these cases.

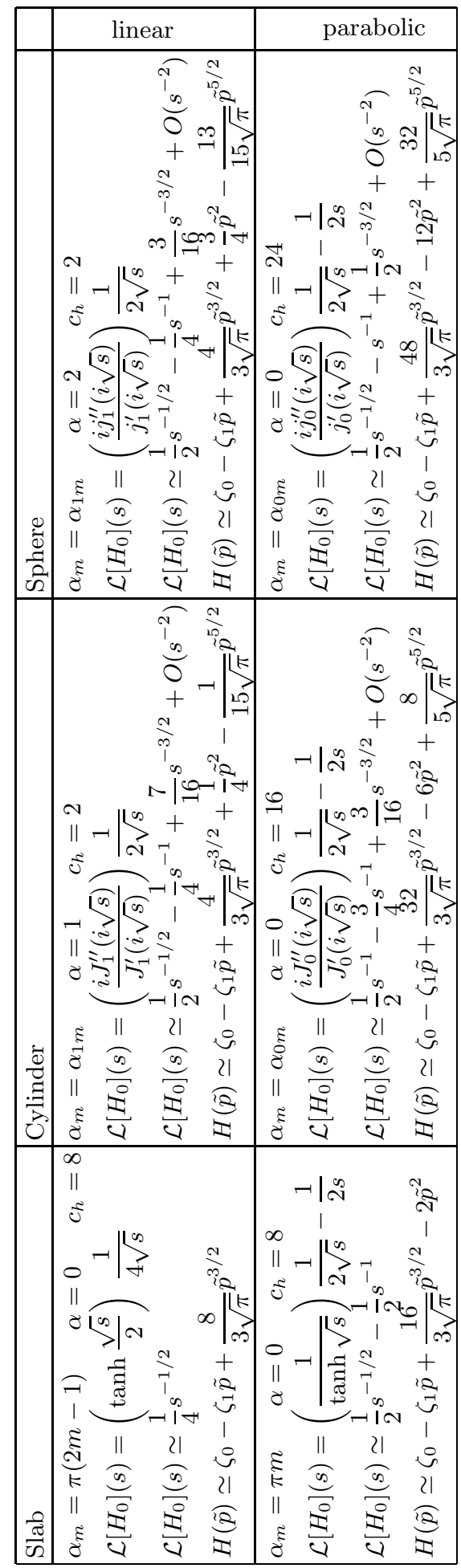

TABLE IV Laplace transforms of the function $H_{0}$ for three basic domains and two choices of the magnetic field spatial profile. The asymptotic behavior of $\mathcal{L}\left[H_{0}\right](s)$ as $s \rightarrow \infty$ allows one to find the correction terms of the function $H(\tilde{p})$ in the slow diffusion limit $p \rightarrow 0$, where $\tilde{p}=p\left(t_{2}-t_{1}\right)$. For a slab geometry, the presented correction terms give very accurate results since higher order corrections decrease exponentially with $s$. Clearly, more accurate results for a cylinder and a sphere involve higher order time averages: $<\left(t_{2}-t_{1}\right)^{2}>_{2}$, $<\left(t_{2}-t_{1}\right)^{5 / 2}>_{2}$, etc. 
- for $m_{1}=m_{2}=m_{3}=0$ (all $\lambda_{m_{i}}$ are zero), the $f$-weighted time average of a constant vanishes;

- when two of three eigenvalues are zero $\left(m_{1}=m_{2}=\right.$ 0 or $m_{1}=m_{3}=0$ or $m_{2}=m_{3}=0$ ), one replaces the remaining exponential function by its leading asymptotic term. The summation over nontrivial eigenvalues gives the same constant for three cases:

$$
\sum_{m=1}^{\infty} \lambda_{m}^{-1} \mathcal{B}_{0, m} \mathcal{B}_{m, 0} \mathcal{B}_{0,0}^{2}=\mathcal{B}_{0,0}^{2} \zeta_{-1}
$$

Then one calculates the $f$-weighted time averages of $\delta$-functions:

$$
\begin{aligned}
& 2<\delta\left(t_{2}-t_{1}\right)>_{4}=2<\delta\left(t_{4}-t_{3}\right)>_{4}= \\
& -<\delta\left(t_{3}-t_{2}\right)>_{4}=\int_{0}^{1} d t f^{2}(t) \hat{f}^{2}(t),
\end{aligned}
$$

where the primitive $\hat{f}$ is defined by (A5). One concludes that the sum of three terms is equal to 0 .

- for the cases $m_{1}=0$ or $m_{3}=0$, one obtains

$$
\begin{aligned}
& <\delta\left(t_{2}-t_{1}\right) \delta\left(t_{3}-t_{2}\right)>_{4}=-\int_{0}^{1} d t f^{3}(t) \hat{f}(t), \\
& <\delta\left(t_{3}-t_{2}\right) \delta\left(t_{4}-t_{3}\right)>_{4}=\int_{0}^{1} d t f^{3}(t) \hat{f}(t),
\end{aligned}
$$

while the spatial averages are identical, so that these contributions cancel each other.

- the only nontrivial case is $m_{2}=0$ (with $m_{1}>0$ and $\left.m_{3}>0\right)$. One has

$$
<\delta\left(t_{2}-t_{1}\right) \delta\left(t_{4}-t_{3}\right)>_{4}=\frac{1}{2}\left(\int_{0}^{1} d t f^{2}(t)\right)^{2}
$$

i.e., the time average of these two delta-functions is factorized. The same happens for the sums over indices $m_{1}$ and $m_{3}$

$$
\sum_{m_{1}, m_{3}} \lambda_{m_{1}}^{-1} \mathcal{B}_{0, m_{1}} \mathcal{B}_{m_{1}, 0} \mathcal{B}_{0, m_{3}} \mathcal{B}_{m_{3}, 0} \lambda_{m_{3}}^{-1}=\zeta_{-1}^{2}
$$

One finally obtains the leading term (108) of the fourth moment in the motional narrowing regime.

\section{APPENDIX D: Parabolic magnetic field}

It has been shown in section $\mathrm{V}$ that the behavior of the second moment $\mathbb{E}\left\{\phi^{2}\right\}$ in the motional narrowing regime is completely determined by a few coefficients $\zeta_{k}$ which depend on the confining geometry and spatial profile of the magnetic field according to (67). Robertson (1966) and Neuman (1974) calculated $\zeta_{-1}$ and $\zeta_{-2}$ for three basic domains (a slab, a cylinder and a sphere) in case of a linear gradient. In subsection V.B, these coefficients were found for restricted diffusion between parallel planes under parabolic magnetic field (55). In this Appendix, we are going to sketch their computation for a cylinder and a sphere.

\section{Cylinder}

Let us first consider a cylinder, for which the matrix $\mathcal{B}$ is given by (L12) in Table I. The only nontrivial contributions to the sums (67) are provided by zeros $\alpha_{m} \equiv \alpha_{0 m}$ of the equation $J_{0}^{\prime}(z)=0$. The relation (L14) in Table I allows one to represent the coefficients $\zeta_{k}$ as

$$
\zeta_{k}=16 \sum_{m=1}^{\infty} \alpha_{m}^{2 k-4}
$$

These sums can be found analytically through so-called Dini's expansions (see (Watson, 1962) for details). In our particular case, one can use the Dini's expansion of the function $x^{2 n}$ over a basis of functions $J_{0}\left(\alpha_{m} x\right)$ on the interval $(0,1)$ :

$$
x^{2 n}=\frac{1}{n+1}+2 \sum_{m=1}^{\infty} c_{m, n} \frac{J_{0}\left(\alpha_{m} x\right)}{J_{0}\left(\alpha_{m}\right)},
$$

where the coefficients $c_{m, n}$ are

$$
c_{m, n}=\frac{1}{J_{0}\left(\alpha_{m}\right)} \int_{0}^{1} d t t^{1+2 n} J_{0}\left(\alpha_{m} t\right) .
$$

The substitution of $n=0$ leads to $c_{m, 0}=0$ for any $m \geq 1$, while the coefficients $c_{m, n}$ for positive $n$ can be expressed by the recurrent formula (Watson, 1962):

$$
c_{m, n}=\frac{2 n}{\alpha_{m}^{2}}-\frac{4 n^{2}}{\alpha_{m}^{2}} c_{m, n-1} .
$$

Applying this formula repeatedly, one finds

$$
c_{m, n}=\sum_{k=0}^{n-1}(-1)^{k} \frac{(n-k) 4^{k}(n !)^{2}}{((n-k) !)^{2}} \alpha_{m}^{-2-2 k} .
$$

Setting $x=1$ in Eq. (D2), one derives for any $n \geq 1$

$$
\frac{n}{2(n+1)}=2 \sum_{k=0}^{n-1}(-1)^{k} \frac{(n-k) 4^{k}(n !)^{2}}{((n-k) !)^{2}} \frac{\zeta_{1-k}}{16} \text {. }
$$

This relation allows one to compute the values of $\zeta_{k}$ for any $k \leq 1$ (for which the corresponding sums are convergent). In particular, one finds the first four values by taking one after another $n=1,2,3,4$ :

$$
\zeta_{1}=2 \quad \zeta_{0}=\frac{1}{12} \quad \zeta_{-1}=\frac{1}{192} \quad \zeta_{-2}=\frac{1}{2880} .
$$

Note that the first two values could be obtained directly by the general relations (68)-(70). 


\section{Sphere}

The computation for a sphere can be performed in a similar way. According to the relation (L14) in Table I, the only nontrivial contributions are provided by the positive roots $\alpha_{m} \equiv \alpha_{0 m}$ of the equation $j_{0}^{\prime}(x)=0$ or $x J_{1 / 2}^{\prime}(x)-\frac{1}{2} J_{1 / 2}(x)=0$ :

$$
\zeta_{k}=24 \sum_{m=1}^{\infty} \alpha_{m}^{2 k-4}
$$

One uses the Dini's expansion

$$
x^{2 n+1 / 2}=\frac{3 x^{1 / 2}}{2 n+3}+2 \sum_{m=1}^{\infty} c_{m, n} \frac{J_{1 / 2}\left(\alpha_{m} x\right)}{J_{1 / 2}\left(\alpha_{m}\right)},
$$

where the coefficients $c_{m, n}$ are

$$
c_{m, n}=\frac{1}{J_{1 / 2}\left(\alpha_{m}\right)} \int_{0}^{1} d t t^{3 / 2+2 n} J_{1 / 2}\left(\alpha_{m} t\right) .
$$

The substitution of $n=0$ leads to $c_{m, 0}=0$ for any $m \geq 1$, while the coefficients $c_{m, n}$ for positive $n$ can be expressed by the recurrent formula (Watson, 1962):

$$
c_{m, n}=\frac{2 n}{\alpha_{m}^{2}}-\frac{4 n(n+1 / 2)}{\alpha_{m}^{2}} c_{m, n-1} .
$$

Taking one after another $n=1,2,3,4$, one derives the coefficients

$\zeta_{1}=\frac{12}{5} \quad \zeta_{0}=\frac{12}{175} \quad \zeta_{-1}=\frac{8}{2625} \quad \zeta_{-2}=\frac{148}{1010625}$.

Again, the first two values could be obtained directly from the general relations (68)-(70).

\section{APPENDIX E: Multiple propagator approach}

A considerable progress in the numerical analysis of diffusive NMR phenomena was achieved by the multiple propagator approach first proposed by Caprihan et al. (1996), further developed by Callaghan (1997) and equivalently reformulated by Sukstanskii and Yablonskiy (2002). Although this approach was intended to study the particular case of a linear magnetic field gradient, we describe its extension for any spatial profile $B(\mathbf{r})$.

Dividing the interval $[0,1]$ into $K$ subintervals of duration $\tau=1 / K$, one can approximate the integral in Eq. (44) by a finite sum

$$
E \simeq \mathbb{E}\left\{\exp \left[i q \tau \sum_{k=0}^{K} f_{k} B\left(X_{k \tau}\right)\right]\right\},
$$

where $f_{k} \equiv f(k \tau)$. To calculate the expectation, all possible values of the random variables $X_{k \tau}$ have to weighted with the corresponding probabilities. Indeed, the starting position $\mathbf{r}_{0}$ is distributed according to a given initial density $\rho\left(\mathbf{r}_{0}\right)$. The probability distribution of the next position $\mathbf{r}_{1}$ after time $\tau$ is given by the Green function $G_{\tau}\left(\mathbf{r}_{0}, \mathbf{r}_{1}\right)$, and so on. One thus obtains

$$
\begin{aligned}
E & \simeq \int_{\Omega} d \mathbf{r}_{0} \int_{\Omega} d \mathbf{r}_{1} \ldots \int_{\Omega} d \mathbf{r}_{K} \rho\left(\mathbf{r}_{0}\right) e^{i q \tau f_{0} B\left(\mathbf{r}_{0}\right)} \times \\
& \times G_{\tau}\left(\mathbf{r}_{0}, \mathbf{r}_{1}\right) e^{i q \tau f_{1} B\left(\mathbf{r}_{1}\right)} G_{\tau}\left(\mathbf{r}_{1}, \mathbf{r}_{2}\right) \ldots e^{i q \tau f_{K} B\left(\mathbf{r}_{K}\right)} .
\end{aligned}
$$

The spectral expansion (25) of the Green function allows to write the signal $E$ as the scalar product $E=(W \cdot H \tilde{W})$, where an infinite-dimension matrix $H$ is

$$
H=R A\left(f_{1}\right) R \ldots A\left(f_{K-1}\right) R .
$$

Two infinite-dimension matrices $R$ and $A$ and two vectors $W$ and $\tilde{W}$ are defined as

$$
\begin{aligned}
A_{m, m^{\prime}}\left(f_{k}\right) & =\int_{\Omega} d \mathbf{r} u_{m}^{*}(\mathbf{r}) u_{m^{\prime}}(\mathbf{r}) \exp \left[i q \tau f_{k} B(\mathbf{r})\right], \\
R_{m, m^{\prime}} & =\delta_{m, m^{\prime}} \exp \left[-p \tau \lambda_{m}\right], \\
W_{m} & =V^{1 / 2} \int_{\Omega} d \mathbf{r} u_{m}(\mathbf{r}) \rho(\mathbf{r}) \exp \left[i q \tau f_{0} B(\mathbf{r})\right], \\
\tilde{W}_{m} & =V^{-1 / 2} \int_{\Omega} d \mathbf{r} u_{m}^{*}(\mathbf{r}) \exp \left[i q \tau f_{K} B(\mathbf{r})\right] .
\end{aligned}
$$

Although the multiple propagator approach provided an efficient numerical tool, its use for theoretical analysis was quite limited since the dependence on the main parameters $p$ and $q$ was hidden in matrices $R$ and $A$ and vectors $W$ and $\tilde{W}$.

One may wonder what happens with the multiple propagator approach in the limit $\tau \rightarrow 0$. In the first order in $\tau$, one has

$$
A\left(f_{k}\right) \simeq I+i q \tau f_{k} \mathcal{B} \quad R \simeq I-p \tau \Lambda,
$$

where the matrices $\Lambda$ and $\mathcal{B}$ are defined by Eqs. (34) and (39) respectively, and $I$ stands for the identity matrix. For sake of simplicity, let us consider the case of reflecting boundaries, for which the elements $W_{m}$ and $\tilde{W}_{m}$ can be simply approximated by $\delta_{m, 0}$. The matrix $H$ becomes

$$
H \simeq \prod_{k=0}^{K}\left[I+\tau\left(i q f_{k} \mathcal{B}-p \Lambda\right)\right]
$$

which can be formally expanded as:

$$
\begin{aligned}
H & \simeq I+\tau \sum_{k_{1}}^{K}\left(i q f_{k_{1}} \mathcal{B}-p \Lambda\right)+\ldots \\
& +\tau^{n} \sum_{k_{1}<\ldots<k_{n}}^{K}\left(i q f_{k_{1}} \mathcal{B}-p \Lambda\right) \ldots\left(i q f_{k_{n}} \mathcal{B}-p \Lambda\right)+\ldots
\end{aligned}
$$


This expansion contains all possible products of matrices $\mathcal{B}$ and $\Lambda$. A general form of the term containing $n$ matrices $\mathcal{B}$ and $m$ matrices $\Lambda$ can be written as

$$
\underbrace{\Lambda \ldots \Lambda}_{\ell_{0}} \mathcal{B} \underbrace{\Lambda \ldots \Lambda}_{\ell_{1}} \mathcal{B} \ldots \underbrace{\Lambda \ldots \Lambda}_{\ell_{n-1}} \mathcal{B} \underbrace{\Lambda \ldots \Lambda}_{\ell_{n}}
$$

where the positive indices $\ell_{0}, \ldots, \ell_{n}$ are such that $\ell_{0}+$ $\ldots+\ell_{n}=m$. The coefficient in front of this term is

$$
(i q)^{n}(-p)^{m} \tau^{n+m} \sum_{k_{1}<\ldots<k_{n+m}}^{K} F_{k_{1}} F_{k_{2}} \ldots F_{k_{n+m}}
$$

where $F_{k_{j}}=f_{k_{j}}$ if the matrix $\mathcal{B}$ stands at $j$-th place in the sequence (E8), and $F_{k_{j}}=1$ otherwise. If the subdivision step $\tau$ is small enough, these coefficients can be considered as integral sums that leads in the limit $\tau \rightarrow 0$ to

$$
\begin{aligned}
(i q)^{n}(-p)^{m} & \int_{0}^{1} d t_{1} \int_{t_{1}}^{1} d t_{2} \ldots \int_{t_{n-1}}^{1} d t_{n} \frac{t_{1}^{\ell_{0}}}{\ell_{0} !} f\left(t_{1}\right) \frac{\left(t_{2}-t_{1}\right)^{\ell_{1}}}{\ell_{1} !} \\
& \times f\left(t_{2}\right) \ldots \frac{\left(t_{n}-t_{n-1}\right)^{\ell_{n-1}}}{\ell_{n-1} !} f\left(t_{n}\right) \frac{\left(1-t_{n}\right)^{\ell_{n}}}{\ell_{n} !} .
\end{aligned}
$$

Taking all possible combinations of indices $\ell_{0}, \ldots, \ell_{n}$ from 0 to infinity, one obtains the $n$-th order contribution to the sum (E7) as

$$
\begin{aligned}
& H_{n}=(i q)^{n} \int_{0}^{1} d t_{1} \int_{t_{1}}^{1} d t_{2} \ldots \int_{t_{n-1}}^{1} d t_{n} f\left(t_{1}\right) \ldots f\left(t_{n}\right) \times \\
& \times e^{-p t_{1} \Lambda} \mathcal{B} e^{-p\left(t_{2}-t_{1}\right) \Lambda} \mathcal{B} \ldots \mathcal{B} e^{-p\left(t_{n}-t_{n-1}\right) \Lambda} \mathcal{B} e^{-p\left(1-t_{n}\right) \Lambda} .
\end{aligned}
$$

According to Eq. (51), the first diagonal element of the matrix $H_{n}$ is exactly the $f$-weighted time average of the correlation function $\mathbb{E}\left\{B\left(X_{t_{1}}\right) \ldots B\left(X_{t_{n}}\right)\right\}$, multiplied by $(i q)^{n}$. Consequently, we retrieved the series expansion (46) for the signal $E$. On the one hand, this derivation reveals how the limit $\tau \rightarrow 0$ can be proceeded within the multiple propagator approach. In particular, it justifies the convergence of this method for $\tau \rightarrow 0$ which was not proved earlier. On the other hand, the direct relation between the multiple propagator approach and the series expansion may be fruitful for further investigation. Note that a very similar analysis allows one to derive the series expansion (46) from the approximate relation (133) in the $\operatorname{limit} \tau \rightarrow 0$.

\section{APPENDIX F: Even order moments in the narrow-pulse approximation}

The compact form (166) of the even order moments can be used to study their dependence on the dimensionless diffusion coefficient $p$. For this purpose, the Green function $G_{T / 2}\left(\mathbf{r}_{1}, \mathbf{r}_{2}\right)$ is represented by the spectral decomposition (25)

$$
\begin{aligned}
& \mathbb{E}\left\{\frac{\phi^{2 n}}{(2 n) !}\right\} \simeq \frac{\delta^{2 n}}{(2 n) !} \sum_{m=0}^{\infty} e^{-p \lambda_{m} / 2} \times \\
& \times \int_{\Omega} d \mathbf{r}_{1} \int_{\Omega} d \mathbf{r}_{2} u_{m}^{*}\left(\mathbf{r}_{1}\right) u_{m}\left(\mathbf{r}_{2}\right)\left(B\left(\mathbf{r}_{1}\right)-B\left(\mathbf{r}_{2}\right)\right)^{2 n}
\end{aligned}
$$

To obtain the leading term, the function $e^{-p \lambda_{m} / 2}$ can be formally developed in a power series up to order $n$ :

$$
\begin{aligned}
& \mathbb{E}\left\{\frac{\phi^{2 n}}{(2 n) !}\right\} \simeq \frac{\delta^{2 n}}{(2 n) !} \sum_{m=0}^{\infty} \sum_{j=0}^{n} \frac{(p / 2)^{j}}{j !} \times \\
& \int_{\Omega} d \mathbf{r}_{1} \int_{\Omega} d \mathbf{r}_{2}(-1)^{j} \lambda_{m}^{j} u_{m}^{*}\left(\mathbf{r}_{1}\right) u_{m}\left(\mathbf{r}_{2}\right)\left(B\left(\mathbf{r}_{1}\right)-B\left(\mathbf{r}_{2}\right)\right)^{2 n} .
\end{aligned}
$$

As earlier, $(-1)^{j} \lambda_{m}^{j}$ can be considered as multiple $(j$ times) application of the Laplace operator to $u_{m}^{*}\left(\mathbf{r}_{1}\right)$. Then the Green formula is used to differentiate by parts. For instance, for $j=1$, one has

$$
\begin{aligned}
& \Delta_{\mathbf{r}_{1}}\left(B\left(\mathbf{r}_{1}\right)-B\left(\mathbf{r}_{2}\right)\right)^{2 n}=2 n(2 n-1)\left(B\left(\mathbf{r}_{1}\right)-B\left(\mathbf{r}_{2}\right)\right)^{2 n-2} \times \\
& \left|\nabla B\left(\mathbf{r}_{1}\right)\right|^{2}+2 n\left(B\left(\mathbf{r}_{1}\right)-B\left(\mathbf{r}_{2}\right)\right)^{2 n-1} \Delta B\left(\mathbf{r}_{1}\right) .
\end{aligned}
$$

The summation over $m$ gives $\delta\left(\mathbf{r}_{1}-\mathbf{r}_{2}\right)$, and the integral vanishes due to the term $\left(B\left(\mathbf{r}_{1}\right)-B\left(\mathbf{r}_{2}\right)\right)^{2 n-2}$ if $2 n-2>$ 0 . In a similar way, all terms vanish for $j<n$. The case $j=n$ leads to the relation (166). A renormalization procedure would be of course required to operate with the "divergent" terms of orders higher than $n$ and to derive the correction terms.

\section{APPENDIX: List of symbols}

$\begin{array}{ll}B_{0} & \text { constant magnetic field } \\ B(\mathbf{r}) & \text { diffusion-sensitizing magnetic field } \\ \mathcal{B}_{m, m^{\prime}} & \text { matrix elements of the magnetic field } \\ & \text { in the Laplace operator eigenbasis, }(39) \\ C_{n}^{k} & \text { binomial coefficients, } C_{n}^{k}=\frac{n !}{(n-k) ! k !} \\ D & \text { free self-diffusion coefficient } \\ D_{a p p} & \text { apparent or effective diffusion coefficient } \\ \mathbb{E} & \text { expectation } \\ E & \text { macroscopic signal (notation } M \\ & \text { can be also found in the literature) } \\ F(t) & \text { auxiliary notation, } f(t) \equiv F(t T) \\ G_{t}\left(\mathbf{r}, \mathbf{r}^{\prime}\right) & \text { Green function in the confining domain } \\ J_{n}(x) & \text { Bessel functions of the first kind } \\ K & \text { surface relaxation rate or permeability }\end{array}$




$\begin{array}{ll}P_{n}(x) & \text { Legendre polynomials } \\ S & \text { total surface area } \\ T & \text { echo time or gradient duration } \\ U, \hat{U} & \text { auxiliary infinite-dimension vectors, }(31),(32) \\ V & \text { volume of the confining domain } \\ X_{t} & \text { reflected Brownian motion, } X_{t} \equiv \mathbf{r}(t T) \\ b & b \text {-value or } b \text {-coefficient, see subsection VI.D } \\ c_{\mathcal{B}} & \text { structure-dependent coefficients, see Table II } \\ c_{m}(t) & \text { time-dependent coefficient in Eq. (123) } \\ d & \text { dimension of the space }(d=3 \text { by default) } \\ f(t) & \text { effective temporal profile of magnetic field } \\ g, g_{1} & \text { gradient intensity } \\ g_{2} & \text { intensity of for the parabolic magnetic field } \\ h & \text { dimensionless relaxation rate } \\ i & \text { imaginary unity, } i^{2}=-1 \\ j_{n}(x) & \text { spherical Bessel functions } \\ \mathfrak{m}(\mathbf{r}) & \text { magnetization, solution of Eq. (9) } \\ p & \text { dimensionless diffusion coefficient, (28) }\end{array}$

$q \quad$ dimensionless magnetic field intensity, (43)

$\mathbf{r}(t) \quad$ stochastic trajectory of a diffusing nucleus

$r \quad$ radial coordinate, radius

$t \quad$ time or dimensionless time

$u_{m}(\mathbf{r}) \quad$ eigenfunctions of the Laplace operator

in the confining domain

$\partial \Omega \quad$ boundary of the confining domain

$\partial / \partial n \quad$ normal derivative at the boundary

(directed towards the bulk)

$\nabla \quad$ gradient

$<\cdot\rangle_{n} \quad f$-weighted time average of a function, (48)

$\prec \cdot \succ_{c} \quad$ cumulant average, see subsection VI.C

! factorial, $n !=1 \cdot 2 \cdot \ldots \cdot n$

$\Gamma(z) \quad$ Euler gamma-function

$\Delta \quad$ Laplace operator, $\Delta=\nabla^{2}$

$\Theta(t) \quad$ Heaviside step function, $\Theta(t)=1$ for $t>0$, and 0 otherwise

$\Lambda \quad$ diagonal matrix formed by eigenvalues $\lambda_{m}$

$\Omega \quad$ confining domain

$\alpha_{m}, \alpha_{n k}$ positive roots of the equations representing the boundary condition, see Table I

$\beta \quad$ magnetic field intensity

$\beta_{m}, \beta_{n k}$ normalization constants for eigenfunctions, see Table I

$\gamma \quad$ gyromagnetic ratio

$\delta \quad$ (normalized) duration of gradient pulses

$\delta_{m, m^{\prime}} \quad$ Kronecker symbol, $\delta_{m, m}=1$, and 0 otherwise

$\delta\left(\mathbf{r}-\mathbf{r}^{\prime}\right)$ Dirac delta-function (distribution)

$\epsilon_{m} \quad$ coefficients, $\epsilon_{0}=1$ and $\epsilon_{m}=\sqrt{2}$ for $m>0$

$\zeta_{k} \quad$ structure-dependent coefficients, (67) $\theta \quad$ azimuthal angle, $0 \leq \theta \leq \pi$

$\lambda_{m}$ dimensionless eigenvalues of the Laplace operator in the confining domain

$\mu \quad$ nuclear magnetic moment

$\mu_{2}$ structure-dependent coefficient, see subsection IV

$\rho(\mathbf{r})$ initial density of nuclei

$\tau \quad$ small time step

$\tau \quad$ ramp time for the trapezoidal profile, Fig. 2(d)

$\phi \quad$ normalized total phase, $\varphi=q \phi$

$\varphi \quad$ total phase of a diffusion nucleus, (1)

$\varphi \quad$ polar angle, $0 \leq \varphi<2 \pi$

\section{References}

Abragam, Anatole, 1961, Principles of Nuclear Magnetism (Oxford University, Oxford).

Arfken, George B., and Hans J. Weber, 2001, Mathematical methods for physicists, 5th Ed. (Academic, San Diego).

Armstrong, R. D., and R. A. Burnham, 1976, "The effect of roughness on the impedance of the interface between a solid electrolyte and a blocking electrode", J. Electroanal. Chem. 72, 257.

Balinov, Balin, Olle Söderman, and Jean-Claude Ravey, 1994, "Diffraction-like Effects Observed in the PGSE Experiment When Applied to a Highly Concentrated Water-Oil Emulsion", J. Phys. Chem. 98, 393.

Balinov, Balin, Bengt Jönsson, Per Linse, and Olle Söderman, 1993, "The NMR Self-Diffusion Method Applied to Restricted Diffusion. Simulation of Echo Attenuation from Molecules in Spheres and Between Planes", J. Magn. Reson. A 104, 17-25 (see also Erratum: J. Magn. Reson. A 108, 130, 1994).

Barbé, René, Michel Leduc, and Franck Lalöe, 1974, "Résonance magnétique en champ de radiofréquence inhomogéne 1re partie: Études théorique", J. Phys. 35, 699725.

Barzykin, Alexandre V., 1998, "Exact Solution of the TorreyBloch Equation for a Spin Echo in Restricted Geometries", Phys. Rev. B 58, 14171.

Barzykin, Alexandre V., 1999, "Theory of Spin Echo in Restricted Geometries under a Step-wise Gradient Pulse Sequence", J. Magn. Reson. 139, 342.

Bass, Richard F., 1998, Diffusions and Elliptic Operators, (Springer, New York).

Bendel, Peter, 1990, "Spin-echo attenuation by diffusion in nonuniform field gradients", J. Magn. Reson. 86, 509.

Bendt, Philip J., 1958, "Measurements of 3He-4He and H2-D2 gas diffusion coefficients", Phys. Rev. 110, 85-89.

Bergman, David J., and Keh-Jim Dunn, 1994, "Theory of Diffusion in a Porous-Medium with Applications to PulsedField Gradient NMR", Phys. Rev. B 50, 9153.

Bergman, David J., and Keh-Jim Dunn, 1995, "NMR of diffusing atoms in a periodic porous medium in the presence of a nonuniform magnetic field", Phys. Rev. E 52, 6516.

Blees, Martin H., 1994, "The Effect of Finite Duration of Gradient Pulses on the Pulsed-Field-Gradient NMR Method for Studying Restricted Diffusion", J. Magn. Res. A 109, 203. 
Bloch, Felix, 1946, "Nuclear Induction", Phys. Rev. 70, 460. Blümich, Bernhard, 2000, NMR Imaging of Materials (Clarendon, Oxford).

Borodin, Andrei N., and Paavo Salminen, 1996, Handbook of Brownian Motion: Facts and Formulae (Birkhauser Verlag, Basel-Boston-Berlin).

Brownstein, Kenneth R., and C. E. Tarr, 1979, "Importance of Classical Diffusion in NMR Studies of Water in Biological Cells", Phys. Rev. A 19 (6), 2446.

Callaghan, Paul T., 1991, Principles of Nuclear Magnetic Resonance Microscopy (Clarendon, Oxford).

Callaghan, Paul T., Andrew Coy, David MacGowan, Ken J. Packer, and Fernando O. Zelaya, 1991, "Diffraction-Like Effects in NMR Diffusion Studies of Fluids in Porous Solids", Nature 351, 467.

Callaghan, Paul T., Andrew Coy, T. P. J. Halpin, David MacGowan, Ken J. Packer, and Fernando O. Zelaya, 1992, "Diffusion in porous systems and the influence of pore morphology in pulsed gradient spin-echo nuclear magnetic resonance studies", J. Chem. Phys. 97, 651-662.

Callaghan, Paul T., Andrew Coy, Lucy C. Forde, and Craig J. Rofe, 1993, "Diffusive relaxation and edge enhancement in NMR microscopy", J. Magn. Reson. A 101, 347-350.

Callaghan, Paul T., 1995, "Pulsed Gradient Spin Echo NMR for planar, cylindrical and spherical pores under conditions of wall relaxation", J. Magn. Reson. A 113, 53-59.

Callaghan, Paul T., and Janez Stepišnik, 1995, "FrequencyDomain Analysis of Spin Motion Using ModulatedGradient NMR", J. Magn. Reson. A 117, 118.

Callaghan, Paul T., and Janez Stepišnik, 1996, "Generalized Analysis of Motions Using Magnetic Field Gradients", Adv. Magn. Opt. Reson. 19, 326.

Callaghan, Paul T., 1997, "A Simple Matrix Formalism for Spin Echo Analysis of Restricted Diffusion under Generalized Gradient Waveforms", J. Magn. Reson. 129, 74.

Callaghan, Paul T., and Sarah L. Codd, 1998, "Generalized Calculation of NMR Imaging Edge Effects Arising from Restricted Diffusion in Porous Media", Magn. Reson. Imag. 16, 471.

Caprihan, Arvind, L. Z. Wang, and Eiichi Fukushima, 1996, "A Multiple-Narrow-Pulse Approximation for Restricted Diffusion in a Time-Varying Field Gradient", J. Magn. Reson. A 118, 94.

Carr, Herman Y., and Edward M. Purcell, 1954, "Effects of diffusion on free precession in NMR experiments", Phys. Rev. 94, 630.

Carslaw, H. S., and J. C. Jaeger, 1959, Conduction of Heat in Solids, 2nd Ed. (Clarendon, Oxford).

Codd, Sarah L., and Paul T. Callaghan, 1999, "Spin Echo Analysis of Restricted Diffusion under Generalized Gradient Waveforms: Planar, Cylindrical and Spherical Pores with Wall Relaxivity", J. Magn. Reson. 137, 358.

Cotts, Robert M., 1991, "Diffusion and diffraction", Nature 351, 443.

Coppens, Marc-Olivier, 1999, "The effect of fractal surface roughness on diffusion and reaction in porous catalysts: from fundamentals to practical application", Catalysis Today 53, 225.

Coy, Andrew, and Paul T. Callaghan, 1994, "Pulsed Gradient Spin Echo nuclear magnetic resonance for molecules diffusing between partially reflecting rectangular barriers", J. Chem. Phys. 101, 4599.

Crank, John, 1975, The Mathematics of Diffusion, 2nd Ed. (Clarendon, Oxford).
Das, T. P., and A. K. Saha, 1954, "Mathematical Analysis of the Hahn Spin-Echo Experiment", Phys. Rev. 93, 749.

de Levie, Robert, 1965, "The Influence of Surface Roughness of Solid Electrodes on Electrochemical Measurements", Electrochimica Acta 10, 113.

de Swiet, Thomas M., and Pabitra N. Sen, 1994, "Decay of nuclear magnetization by bounded diffusion in a constant field gradient", J. Chem. Phys. 100, 5597.

Douglass, Dean. C., and David W. McCall, 1958, "Diffusion in Paraffin Hydrocarbons", J. Phys. Chem. 62, 1102.

Duh, Andrej, Aleš Mohorič, and Janez Stepišnik, 2001, "Computer Simulation of the Spin-Echo Signal Distribution in the Case of Restricted Self-Diffusion", J. Magn. Reson. 148, 257.

Felici, Maddalena, Marcel Filoche, and Bernard Sapoval, 2004, "Renormalized Random Walk Study of Oxygen Absorption in the Human Lung", Phys. Rev. Lett. 92, 068101.

Felici, Maddalena, Marcel Filoche, C. Straus, T. Similowski, and Bernard Sapoval, 2005, "Diffusional Screening in Real 3D Human Acini - a Theoretical Study", Resp. Physiol. Neurobiol. 145, 279.

Feller, William, 1971, An Introduction to Probability Theory and Its Applications, Volumes I and II, Second Edition (John Wiley \& Sons, New York).

Filoche, Marcel, and Bernard Sapoval, 1999, "Can One Hear the Shape of an Electrode? II. Theoretical Study of the Laplacian Transfer", Eur. Phys. J. B 9, 755-763.

Fordham, E. J., Partha P. Mitra, and L. L. Latour, 1996, "Effective Diffusion Times in Multiple-Pulse PFG Diffusion Measurements in Porous Media" J. Magn. Reson. A, 121, 187-192.

Freidlin, Mark, 1985, "Functional Integration and Partial Differential Equations", Annals of Mathematics Studies (Princeton University, Princeton, New Jersey).

Frøhlich, Astrid F., Leif Østergaard, and Valerij G. Kiselev, 2006, "Effect of Impermeable Boundaries on DiffusionAttenuated MR Signal", J. Magn. Reson. (in press).

Gibbs, Stephen J., 1997, "Observations of diffusive diffraction in a cylindrical pore by PFG NMR", J. Magn. Reson. 124, 223.

Grebenkov, Denis S., Marcel Filoche, and Bernard Sapoval, 2003, "Spectral Properties of the Brownian Self-Transport Operator", Eur. Phys. J. B 36 (2), 221-231.

Grebenkov, Denis S., Marcel Filoche, Bernard Sapoval, and Maddalena Felici, 2005, "Diffusion-Reaction in Branched Structures: Theory and Application to the Lung Acinus", Phys. Rev. Lett. 94, 050602.

Grebenkov, Denis S., 2006, "Partially Reflected Brownian Motion: A Stochastic Approach to Transport Phenomena", in Focus on Probability Theory, Ed. F. Columbus, Vol. 2 (Nova Science Publishers Inc., in press).

Grebenkov, Denis S., 2006, "Multiexponential attenuation of the CPMG spin echoes due to a geometrical confinement", J. Magn. Reson. 179, 393-401.

Grebenkov, Denis S., Marcel Filoche, and Bernard Sapoval, 2006, "Mathematical Basis for a General Theory of Laplacian Transport towards Irregular Interfaces", Phys. Rev. E 73, 021103.

Grebenkov, Denis S., and Geneviève Guillot, 2006, "Localization regime of restricted diffusion in a model pulmonary acinus", in Proceedings of the Fourteenth ISMRM Meeting, \#2793 (Seattles).

Grebenkov, Denis S., 2006, "Scaling Properties of the Spread Harmonic Measures", Fractals (accepted). 
Hahn, Erwin L., 1950, "Spin Echoes", Phys. Rev. 80 (4), 580. Halperin, William P., Jyh-Yuar Jehng, and Yi-Qiao Song, 1994, "Application of spin-spin relaxation to measurement of surface area and pore size distributions in a hydrating cement paste", Magn. Reson. Imaging 12, 169.

Halsey, Thomas C., and Michael Leibig, 1992, "The Double Layer Impedance at a Rough Surface: Theoretical Results", Annals of Physics 219, 109.

Hayden, Mike E., G. Archibald, K. M. Gilbert, and C. Lei, 2004, "Restricted diffusion within a single pore", J. Magn. Reson. 169, 313.

Helmer, Karl G., Martin D. Hürlimann, Thomas M. de Swiet, Pabitra N. Sen, and Christopher H. Sotak, 1995, "Determination of ratio of surface area to pore volume from restricted diffusion in a constant field gradient", J. Magn. Reson. A 115, 257-259.

Helmer, Karl G., Bernard J. Dardzinski, and Christopher H. Sotak, 1995, The Application of Porous-Media Theory to the Investigation of Time-Dependent Diffusion in In Vivo Systems, NMR in Biomedicine 8, 297-306.

Hürlimann, Martin D., Karl G. Helmer, L. L. Latour, and Christopher H. Sotak, 1994, "Restricted Diffusion in Sedimentary Rocks. Determination of Surface-Area-to-Volume Ratio and Surface Relaxivity", J. Magn. Reson. A 111, 169-178.

Hürlimann, Martin D., Karl G. Helmer, Thomas M. de Swiet, Pabitra N. Sen, and Christopher H. Sotak, 1995, "Spin Echoes in a Constant Gradient and in the Presence of Simple Restriction", J. Magn. Reson. A 113, 260.

Hürlimann, Martin D., 1998, "Effective Gradients in Porous Media Due to Susceptibility Differences", J. Magn. Reson. 131, 232.

Hürlimann, Martin D., 2001, "Diffusion and relaxation effects in general stray field NMR experiments", J. Magn. Reson. 148, 367.

Hyslop, W. Brian, and Paul C. Lauterbur, 1991, "Effects on restricted diffusion on microscopic NMR imaging", J. Magn. Reson. 94, 501-510.

Itô, Kiyosi, and Henry P. McKean, 1965, Diffusion Processes and Their Sample Paths (Springer-Verlag, Berlin).

Kac, Mark, 1966, "Can One Hear the Shape of a Drum?", Am. Math. Monthly 73, 1.

Kaplan, Jerome I., 1959, "Application of the DiffusionModified Bloch Equation to Electron Spin Resonance in Ordinary and Ferromagnetic Metals", Phys. Rev. 115, 575.

Kimmich, Rainer, 1997, NMR - Tomography, Diffusometry, Relaxometry (Springer-Verlag, Berlin Heidelberg, New York).

Kimmich, Rainer, 2002, "Strange kinetics, porous media, and NMR", Chem. Phys. 284, 253.

King, Martin D., John Houseman, Simon A. Roussel, Nicholas van Bruggen, Stephen R. Williams, and David G. Gadian, 1994, "q-Space imaging of the brain", Magn. Reson. Med. 32, 707.

Kiselev, Valerij G., and S. Posse, 1998, "Analytical Theory of Susceptibility Induced NMR Signal Dephasing in a Cerebrovascular Network", Phys. Rev. Lett. 81, 5696.

Kiselev, Valerij G., and Dmitry S. Novikov, 2002, "Transverse NMR Relaxation as a Probe of Mesoscopic Structure", Phys. Rev. Lett. 89, 278101.

Klafter, J., and J. M. Drake (Eds.), 1989, "Molecular Dynamics in Restricted Geometries" (John Wiley and Sons, New York).

Kleinberg, Robert L., and Mark A. Horsfield, 1990, "Trans- verse relaxation processes in porous sedimentary rock", J. Magn. Reson. 88, 9.

Kleinberg, Robert L., A. Sezginer, D. D. Griffin, and M. Fukuhara, 1992, "Novel NMR apparatus for investigating an external sample", J. Magn. Reson. 97, 466.

Kuchel, Philip W., Alison J. Lennon, and Christopher Durrant, 1996, "Analytical Solutions and Simulations for SpinEcho Measurements of Diffusion of Spins in a Sphere with Surface and Bulk Relaxation", J. Magn. Reson. B 112, 1.

Latour, L. L., Partha P. Mitra, Robert L. Kleinberg, and Christopher H. Sotak, 1993, "Time-dependent diffusion coefficient of fluids in porous media as a probe of surface-tovolume ratio", J. Magn. Reson. A 101, 342-346.

Latour, L. L., Robert L. Kleinberg, Partha P. Mitra, and Christopher H. Sotak, 1995, "Pore-Size Distributions and Tortuosity in Heterogeneous Porous Media", J. Magn. Reson. A, 112, 83-91.

Le Bihan, Denis, 2003, "Looking into the functional architecture of the brain with diffusion MRI", Nature Reviews Neurosci. 4 (6), 469-480.

Le Doussal, Pierre, and Pabitra N. Sen, 1992, "Decay of Nuclear Magnetization by Diffusion in a Parabolic Magnetic Field: an Exactly Solvable Model", Phys. Rev. B 46, 3465.

Linse, Per, and Olle Söderman, 1995, "The validity of the short-gradient-pulse approximation in NMR studies of restricted diffusion. Simulations of molecules diffusing between planes, in cylinders and spheres", J. Magn. Reson. A 116, 77 .

Lori, Nicolas F., Thomas E. Conturo, and Denis Le Bihan, 2003, "Definition of Displacement Probability and Diffusion Time in q-Space Magnetic Resonance Measurements That Use Finite-Duration Diffusion-Encoding Gradients", J. Magn. Reson. 165, 185.

Mair, Ross W., David G. Cory, Sharon Peled, Ching-Hua Tseng, Samuel Patz, and Ronald L. Walsworth, 1998, "Pulsed-field-gradient measurements of time-dependent gas diffusion" J. Magn. Reson. 135, 478-486.

Mair, Ross W., G. P. Wong, D. Hoffmann, Martin D. Hürlimann, Samuel Patz, Lawrence M. Schwartz, and Ronald L. Walsworth, 1999, "Probing Porous Media with Gas Diffusion NMR", Phys. Rev. Lett. 83, 3324.

Mair, Ross W., Pabitra N. Sen, Martin D. Hürlimann, Samuel Patz, David G. Cory, and Ronald L. Walsworth, 2002, "The Narrow Pulse Approximation and Long Length Scale Determination in Xenon Gas Diffusion NMR Studies of Model Porous Media", J. Magn. Reson. 156, 202.

Mair, Ross W., Matthew S. Rosen, R. Wang, David G. Cory, and Ronald L. Walsworth, 2002, "Diffusion NMR methods applied to xenon gas for materials study", Magn. Reson. Chem. 40, S29.

Majumdar, S., and J. C. Gore, 1988, "Studies of diffusion in random fields produced by variations in susceptibility", J. Mang. Reson. 78, 41.

Malmborg, Carin, Daniel Topgaard, and Olle Söderman, 2004, "NMR Diffusometry and the Short Gradient Pulse Limit Approximation", J. Magn. Reson. 169, 85.

Meiboom, S., and D. Gill, 1958, "Modified Spin-Echo Method for Measuring Nuclear Relaxation Times", Rev. Sci. Instrum. 29, 688 .

Mitra, Partha P., and Pierre Le Doussal, 1991, "Long-time magnetization relaxation of spins diffusing in a random field", Phys. Rev. B 44, 12035.

Mitra, Partha P., Pabitra N. Sen, Lawrence M. Schwartz, and Pierre Le Doussal, 1992, "Diffusion propagator as a probe 
of the structure of porous media", Phys. Rev. Lett. 68, 3555 .

Mitra, Partha P., and Pabitra N. Sen, 1992, "Effects of microgeometry and surface relaxation on NMR pulsed-fieldgradient experiments: Simple pore geometries", Phys. Rev. B 45, 143.

Mitra, Partha P., Pabitra N. Sen, and Lawrence M. Schwartz, 1993, "Short-time behavior of the diffusion coefficient as a geometrical probe of porous media", Phys. Rev. B 47, 8565.

Mitra, Partha P., and Bertrand I. Halperin, 1995, "Effects of finite gradient pulse widths in pulsed field gradient diffusion measurements", J. Magn. Reson. A 113, 94.

Mitra, Partha P., L. L. Latour, Robert L. Kleinberg, and Christopher H. Sotak, 1995, "Pulsed-Field-Gradient NMR Measurements of Restricted Diffusion and the Return-tothe-Origin Probability", J. Magn. Reson. A, 114, 47-58.

Möller, Harald E., X. Josette Chen, Brian Saam, Klaus D. Hagspiel, G. Allan Johnson, Talissa A. Altes, Eduard E. de Lange, and Hans-Ulrich Kauczor, 2002, "MRI of the lungs using hyperpolarized noble gases", Magn. Reson. Med. 47, 1029-1051.

Murday, J. S., and Robert M. Cotts, 1968, "Self-Diffusion Coefficient of Liquid Lithium", J. Chem. Phys. 48, 4938.

Nestle, Nikolaus, Petrik Galvosas, Oliver Geier, Marwan Dakkouri, Christian Zimmermann, and J. Kärger, 2001, "NMR studies of water diffusion and relaxation in hydrating slag-based construction materials", Magn. Reson. Imaging 19, 547 .

Neuman, C. H., 1974, "Spin echo of spins diffusion in a bounded medium", J. Chem. Phys. 60, 4508-4511.

Nicholson, Charles, 1985, "Diffusion from an Injected Volume of a Substance in Brain-Tissue with Arbitrary Volume Fraction and Tortuosity", Brain Research 333 (2), 325-329.

Nicholson, Charles, 2001, "Diffusion and Related Transport Mechanisms in Brain Tissue", Rep. Progr. Phys. 64 (7), 815-884.

Nyikos, Lajos, and Tamás Pajkossy, 1985, "Fractal Dimension and Fractional Power Frequency-Dependent Impedance of Blocking Electrodes", Electrochimica Acta 30, 1533.

Nyikos, Lajos, and Tamás Pajkossy, 1986, "Diffusion to Fractal Surfaces", Electrochimica Acta 31 (10), 1347.

Plassais, Arnaud, Marie-Pierre Pomies, Nicolas Lequeux, Philippe Boch, Jean-Pierre Korb, Dominique Petit, and Fabien Barberon, 2003, "Micropore size analysis by NMR in hydrated cement", Magn. Reson. Imaging 21, 369.

Port, Sidney C., Charles J. Stone, 1978, Brownian Motion and Classical Potential Theory, (Academic Press, New York).

Price, William S., Peter Stilbs, and Olle Söderman, 2003, "Determination of Pore Space Shape and Size in Porous Systems Using NMR Diffusometry. Beyond the Short Gradient Pulse Approximation", J .Magn. Reson. 160, 139.

Pütz, B., D. Barsky, and K. Schulten, 1992, "Edge enhancement by diffusion in microscopic MRI", J. Magn. Reson. 97, 27-53.

Revuz, Daniel R. J., and Marc Yor, 1999, Continuous Martingales and Brownian Motion (Springer, Berlin).

Robertson, Baldwin, 1966, "Spin-echo decay of spins diffusion in a bounded region", Phys. Rev. 151, 273-277.

Saam, Brian T., Dmitriy A. Yablonskiy, Vikram D. Kodibagkar, Jason C. Leawoods, David S. Gierada, Joel D. Cooper, Stephen S. Lefrak, and Mark S. Conradi, 2000, "MR imaging of diffusion of $3 \mathrm{He}$ gas in healthy and diseased lungs", Magn. Reson. Med. 44, 174-179.

Sahimi, Muhammad, George R. Gavalas, and Theodore T.
Tsotsis, 1990, "Statistical and continuum models of fluidsolid reactions in porous media", Chem. Engng. Sci. 45, 1443.

Sapoval, Bernard, 1987, "Fractal Electrodes and Constant Phase Angle Response: Exact Exemples and Counter Examples", Solid State Ionics 23, 253.

Sapoval, Bernard, 1994, "General Formulation of Laplacian Transfer Across Irregular Surfaces", Phys. Rev. Lett. 73, 3314 .

Sapoval, Bernard, 1996, "Transport Across Irregular Interfaces: Fractal Electrodes, Membranes and Catalysts", in Fractals and Disordered Systems, Eds. A. Bunde, S. Havlin, 233-261 (Springer).

Schwartz, Lawrence M., Martin D. Hürlimann, Keh-Jim Dunn, Partha P. Mitra, and David J. Bergman, 1997, "Restricted diffusion and the return to the origin probability at intermediate and long times", Phys. Rev. E 55, 4225-4234.

Sen, Pabitra N., Lawrence M. Schwartz, Partha P. Mitra, and Bertrand I. Halperin, 1994, "Surface relaxation and the long-time diffusion coefficient in porous media: periodic geometries", Phys. Rev. B 49, 215.

Sen, Pabitra N., Martin D. Hürlimann, and Thomas M. de Swiet, 1995, "Debye-Porod law of diffraction for diffusion in porous media", Phys. Rev. B 51, 601.

Sen, Pabitra N., Alex André, and Scott Axelrod, 1999, "Spin echoes of nuclear magnetization diffusing in a constant magnetic field gradient and in a restricted geometry", J. Chem. Phys. 111, 6548.

Söderman, Olle, and Bengt Jönsson, 1995, "Restricted Diffusion in Cylindrical Geometry", J. Magn. Reson. A 117, 94.

Song, Yi-Qiao, 2000, "Detection of the High Eigenmodes of Spin Diffusion in Porous Media", Phys. Rev. Lett. 85, 3878.

Song, Yi-Qiao, Seungoh Ryu, and Pabitra N. Sen, 2000, "Determining multiple length scales in rocks", Nature (London) 406, 178.

Song, Yi-Qiao, Martin D. Hürlimann, and C. Flaum, 2003, "A Method for Rapid Characterization of Diffusion", J. Magn. Reson. 161, 222.

Stejskal, E. O., and J. E. Tanner, 1965, "Spin diffusion measurements: spin echoes in the presence of a time-dependent field gradient", J. Chem. Phys. 42, 288.

Stejskal, E. O., 1965, "Use of Spin Echoes in a Pulsed Magnetic-Field Gradient to Study Anisotropic, Restricted Diffusion and Flow", J. Chem. Phys. 43, 3597.

Stepišnik, Janez, 1981, "Analysis of NMR self-diffusion measurements by density matrix calculation", Physica B 104, 350.

Stepišnik, Janez, 1985, "Measuring and imaging of flow by NMR", Prog. Nucl. Magn. Reson. Spectrosc. 17, 187.

Stepišnik, Janez, 1993, "Time-dependent self-diffusion by NMR spin-echo", Physica B 183, 343.

Stepišnik, Janez, 1998, "Spin Echo Attenuation of Restricted Diffusion as a Discord of Spin Phase Structure", J. Magn. Reson. 131, 339.

Stepišnik, Janez, 1999, "Validity Limits of Gaussian Approximation in Cumulant Expansion for Diffusion Attenuation of Spin Echo", Physica B 270, 110.

Stoller, S. D., William Happer, and Freeman J. Dyson, 1991, "Transverse spin relaxation in inhomogeneous magnetic fields", Phys. Rev. A 44, 7459.

Sukstanskii, Alexander L., and Dmitriy A. Yablonskiy, 2002, "Effects of Restricted Diffusion on MR Signal Formation", J. Magn. Reson. 157, 92. 
Sukstanskii, Alexander L., and Dmitriy A. Yablonskiy, 2003, "Gaussian approximation in the theory of MR signal formation in the presence of structure-specific magnetic field inhomogeneities", J. Magn. Reson. 163, 236.

Sukstanskii, Alexander L., and Dmitriy A. Yablonskiy, 2004, "Gaussian approximation in the theory of MR signal formation in the presence of structure-specific magnetic field inhomogeneities. Effects of impermeable susceptibility inclusions", J. Magn. Reson. 167, 56.

Tanner, J. E., and E. O. Stejskal, 1968, "Restricted SelfDiffusion of Protons in Colloidal Systems by the PulsedGradient, Spin-Echo Method", J. Chem. Phys. 49, 1768.

Tarczon, John C., and William P. Halperin, 1985, "Interpretation of NMR diffusion measurements in uniform- and nonuniform-field profiles", Phys. Rev. B 32, 2798.

Torrey, Henry C., 1956, "Bloch Equations with Diffusion Terms", Phys. Rev. 104, 563.

van Beek, Edwin J. R., Jim M. Wild, Hans-Ulrich Kauczor, Wolfgang Schreiber, John P. Mugler III, and Eduard E. de Lange, 2004, "Functional MRI of the Lung Using Hyperpolarized 3-Helium Gas", J. Magn. Reson. Imag. 20, 540-554.

Wang, L. Z., Arvind Caprihan, and Eiichi Fukushima, 1995, "The narrow-pulse criterion for pulsed-gradient spin-echo diffusion measurements", J. Magn. Reson. A 117, 209.

Watson, George N., 1962, A Treatise on the Theory of Bessel Functions (Cambridge University, Cambridge).

Wayne, R. C., and Robert M. Cotts, 1966, "Nuclearmagnetic-resonance study of self-diffusion in a bounded medium", Phys. Rev. 151, 264.

Weibel, Ewald R., 1984, "The Pathway for oxygen. Structure and function in the mammalian respiratory system" (Har- vard University, Cambridge, Massachusetts and London, England).

Woessner, Donald E., 1960, "Self-Diffusion Measurements in Liquids by the Spin-Echo Technique", Rev. Sci. Instr. 31, 1146.

Woessner, Donald E., 1961, "Effects of diffusion in nuclear magnetic resonance spin-echo experiments", J. Chem. Phys. 34, 2057.

Woessner, Donald E., 1963, "NMR spin-echo self-diffusion measurements on fluids undergoing restricted diffusion", J. Phys. Chem. 67, 1365.

Yablonskiy, Dmitriy A., Alexander L. Sukstanskii, Jason C. Leawoods, David S. Gierada, G. Larry Bretthorst, Stephen S. Lefrak, Joel D. Cooper, and Mark S. Conradi, 2002, "Quantitative in vivo Assessment of Lung Microstructure at the Alveolar Level with Hyperpolarized $3 \mathrm{He}$ Diffusion MRI", PNAS 99 (5), 3111-3116.

Yor, Marc, 2001, Exponential Functionals of Brownian Motion and Related Processes, (Springer, Berlin).

Zhang, Gigi Q., and George J. Hirasaki, 2003, "CPMG relaxation by diffusion with constant magnetic field gradient in a restricted geometry: numerical simulation and application", J. Magn. Reson. 163, 81.

Zielinski, Lukasz J., and Pabitra N. Sen, 2000, "Relaxation of nuclear magnetization in a nonuniform magnetic field gradient and in a restricted geometry", J. Magn. Reson. 147, 95.

Zielinski, Lukasz J., and Martin D. Hürlimann, 2005, "Probing short length scales with restricted diffusion in a static gradient using the CPMG sequence", J. Magn. Reson. 172, 161-167. 